\title{
Practical Oxygen Carrier Development of Unique Trimetallic Oxide for Chemical Looping Combustion of Carbonaceous Fuels
}

\author{
William J Benincosa III
}

Follow this and additional works at: https://researchrepository.wvu.edu/etd

\section{Recommended Citation}

Benincosa, William J III, "Practical Oxygen Carrier Development of Unique Trimetallic Oxide for Chemical Looping Combustion of Carbonaceous Fuels" (2018). Graduate Theses, Dissertations, and Problem Reports. 7161.

https://researchrepository.wvu.edu/etd/7161

This Dissertation is protected by copyright and/or related rights. It has been brought to you by the The Research Repository @ WVU with permission from the rights-holder(s). You are free to use this Dissertation in any way that is permitted by the copyright and related rights legislation that applies to your use. For other uses you must obtain permission from the rights-holder(s) directly, unless additional rights are indicated by a Creative Commons license in the record and/ or on the work itself. This Dissertation has been accepted for inclusion in WVU Graduate Theses, Dissertations, and Problem Reports collection by an authorized administrator of The Research Repository @ WVU.

For more information, please contact researchrepository@mail.wvu.edu. 


\title{
Practical Oxygen Carrier Development of Unique Trimetallic Oxide for Chemical Looping Combustion of Carbonaceous Fuels
}

\author{
William J. Benincosa III \\ Dissertation submitted \\ to the College of Engineering and Mineral Resources \\ at West Virginia University \\ in partial fulfillment of the requirements for the degree of \\ Doctor of Philosophy in \\ Chemical and Biomedical Engineering \\ Hanjing Tian, Ph.D., Chair \\ John Hu, Ph.D. \\ John Zondlo, Ph.D. \\ Xingbo Liu, Ph.D. \\ Dushyant Shekhawat, Ph.D. \\ Ranjani Siriwardane, Ph.D. \\ Chemical and Biomedical Engineering \\ Morgantown, West Virginia \\ 2018
}

Keywords: Oxygen Carrier Development, Chemical Looping Combustion, $\mathrm{CO}_{2}$ Capture Copyright 2018 William J. Benincosa III 


\title{
ABSTRACT \\ Practical Oxygen Carrier Development of Unique Trimetallic Oxide for Chemical Looping Combustion of Carbonaceous Fuels
}

\author{
William J. Benincosa III
}

Chemical Looping Combustion (CLC) is a cyclical process which provides oxygen in the form of an oxygen carrier, or metal oxide, rather than air to combust the fuel source for power generation and produce a pure stream of sequestration-ready carbon dioxide $\left(\mathrm{CO}_{2}\right)$. Successful implementation of CLC depends highly on the choice of oxygen carrier as this has been deemed the major barrier to the progression this technology. The criteria for a successful oxygen carrier is four-fold. The carrier must be (1) highly reactive, (2) environmentally friendly, (3) low-cost, and (4) possess chemical and physical stability. Most carriers cited in literature do not meet the stringent requirements necessary for further investigation.

In this dissertation, a promising trimetallic copper iron manganese oxygen carrier was developed in fulfillment of the oxygen carrier criteria. The results showed that formulation played a vital role in chemical reactivity and physical strength. The formation a unique phase was confirmed with DSC/TGA experiments and characterized further with SEM-EDS, DSC/TGA, XPS, and in-situ XRD to gain a clear elucidation of the oxygen carrier during chemical looping combustion reactions with carbonaceous fuels such as methane $\left(\mathrm{CH}_{4}\right)$, and components of coal derived synthesis gas, hydrogen $\left(\mathrm{H}_{2}\right)$ and carbon monoxide $(\mathrm{CO})$. Also, experimental data was collected with TGA-MS and analyzed with established solid-state mathematical models using curve-fitting software to develop valuable reaction parameters such as reaction activation energy, frequency factor, and intrinsic rate constants for reactor design and scale-up.

Despite the notion that a high-performing oxygen carrier does not exist, this study found that the trimetallic oxygen carrier could hurdle the last challenge in CLC implementation, which is the selection of the ideal oxygen carrier. 


\section{Dedication}

To my family, friends, classmates, and colleagues who have given their selfless support throughout my doctoral education. 


\section{Acknowledgements}

I would like to extend my sincere gratitude to Dr. Hanjing Tian for his guidance throughout my endeavor at WVU.

I would like to thank my mentor Dr. Ranjani Siriwardane for providing an extensive background in scientific research and problem solving.

I would like to thank my committee members, Dr. John Zondlo, Dr. John Hu, Dr. Xingbo Liu, and Dr. Dushyant Shekhawat for their time and contribution to my doctoral education.

I would like to thank WVU and USDOE/NETL for sponsoring my doctoral education and research activities.

I would like to thank Jarrett Riley, Daniel Haynes, James Poston, and Richard Eddy for their support and suggestions.

I would like to thank my friends and family for always believing in me, and Erika Brooks for her constant motivation throughout this process. 


\section{Table of Contents}

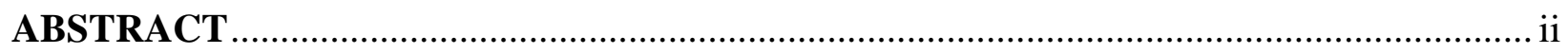

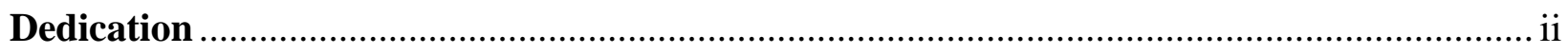

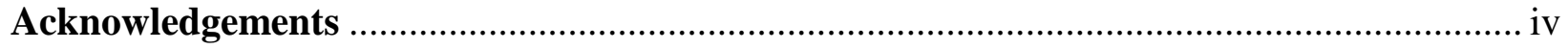

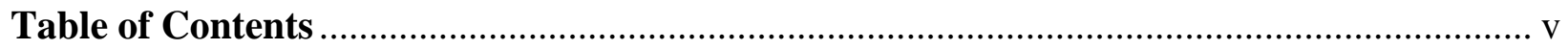

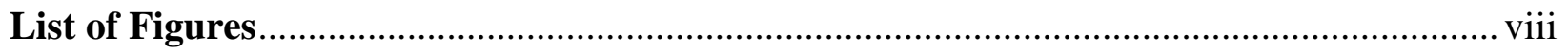

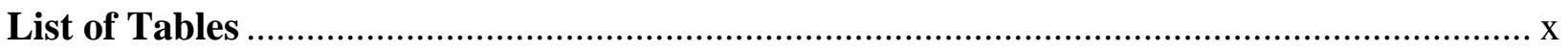

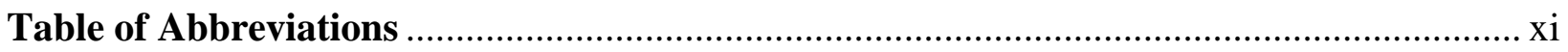

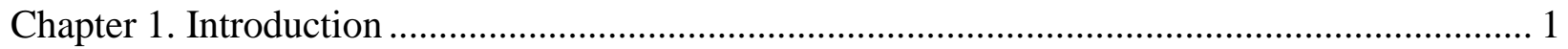

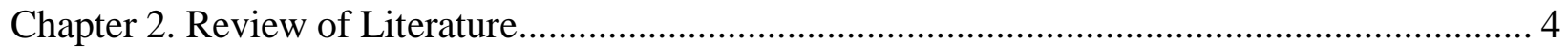

2.1. Chemical Looping Combustion …………………………………………………….... 4

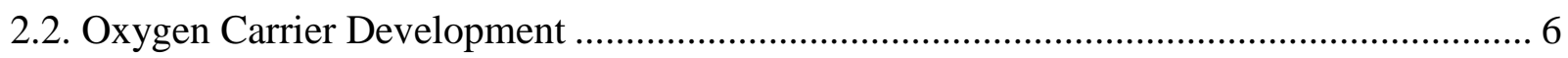

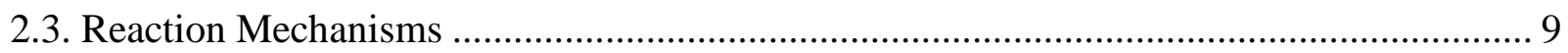

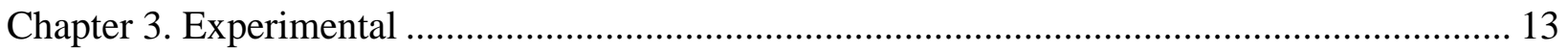

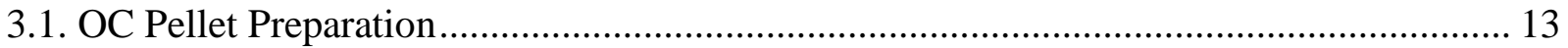

3.2. Reduction/ Oxidation TGA with Online Effluent Gas Analysis by Mass Spectrometry

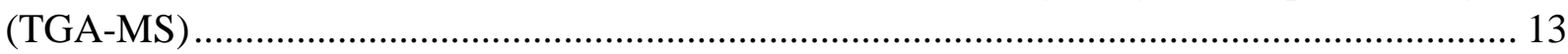

3.3. Micromeritics Fixed Bed Reactor with Online Effluent Gas Analysis by Mass Spectrometry (FB-MS) ................................................................................................ 13

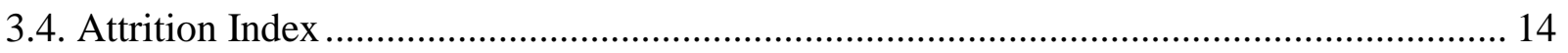

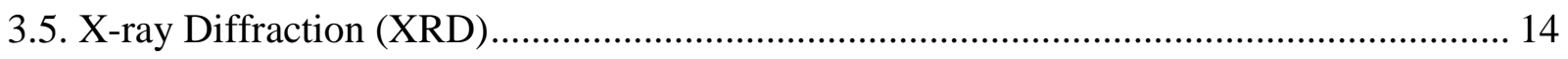

3.6. Simultaneous Differential Scanning Calorimetry and Thermogravimetric Analysis during Temperature Programmed Reduction (DSC/TGA TPR) …………………………............ 15

3.7. Brunauer-Emmett-Teller (BET) Analysis ................................................................... 16

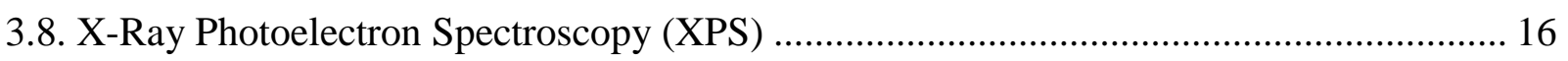

3.9. Scanning Electron Microscopy with Energy Dispersive Spectroscopy (SEM-EDS) ........ 17

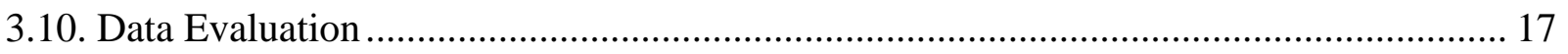

3.11. FactSage Calculations …………………………..................................................... 18

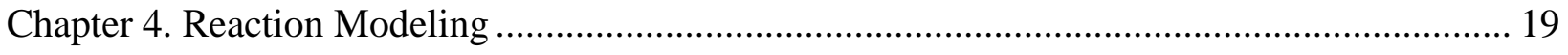

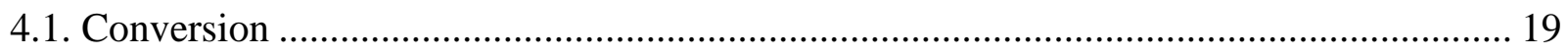

4.2. Chemical and Diffusion Reaction Controls ................................................................. 19

4.3. Nucleation and Growth ............................................................................................ 23

4.4. Model-Fitting and Model-Free Analysis..................................................................... 24 
Chapter 5. Design of a High Performing Oxygen Carrier

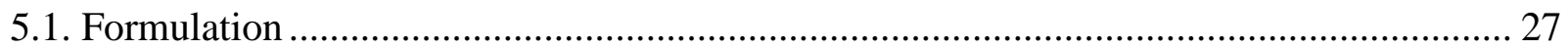

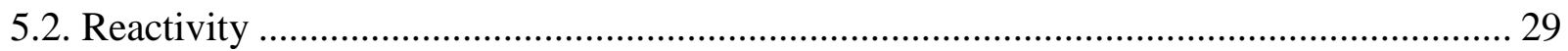

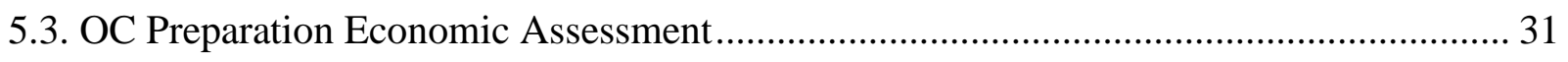

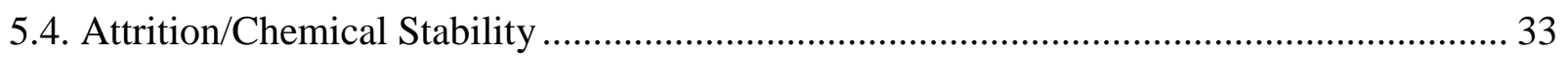

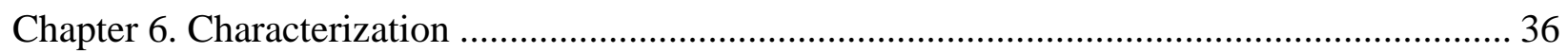

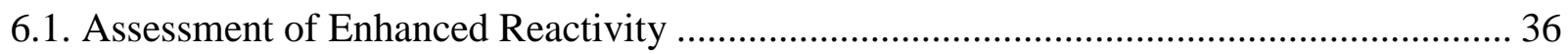

6.2. DSC/TGA during TPR with $\mathrm{CH}_{4}$ Performance Data Comparison of $\mathrm{CuFeMnO}_{4}$ and Corresponding Pure Metal Oxides ................................................................................ 38

6.3. DSC/TGA during TPR with $\mathrm{CH}_{4}$ Performance Data Comparison of $\mathrm{CuFeMnO}_{4}$ and

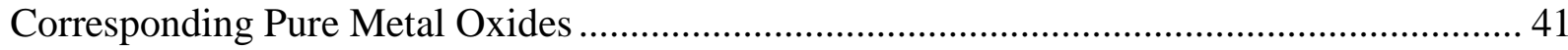

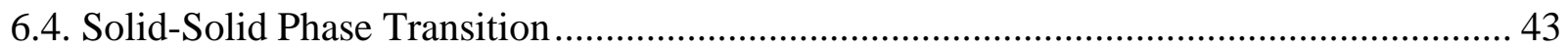

Chapter 7. Reaction Characterization and Kinetic Modeling during the Reduction of $\mathrm{CuFeMnO}_{4}$ with $\mathrm{H}_{2}$

7.1. DSC/TGA during TPR with $\mathrm{H}_{2}$ Performance Data Comparison of $\mathrm{CuFeMnO}_{4}$ and

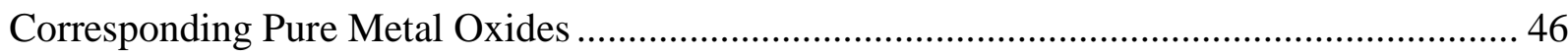

7.2. DSC/TGA during TPR with $\mathrm{H}_{2}$ Performance Data Comparison of $\mathrm{CuFeMnO}_{4}$ and

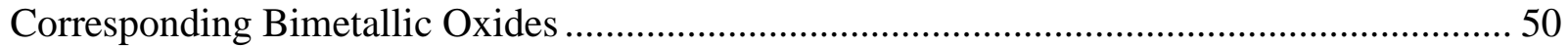

7.3. In-situ XRD TPR with 5\% $\mathrm{H}_{2}$ Analysis ................................................................. 53

7.4. In-situ XRD Temperature Programmed Oxidation (TPO) with 100\% Air Analysis ......... 55

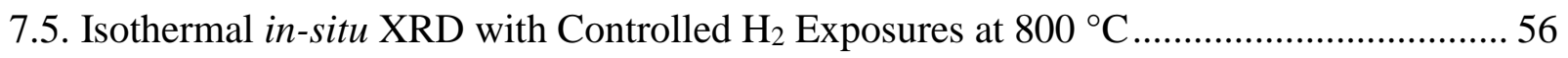

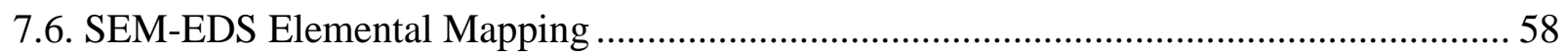

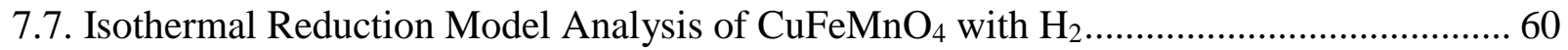

Chapter 8. Reaction Characterization and Kinetic Modeling during the Reduction of $\mathrm{CuFeMnO}_{4}$

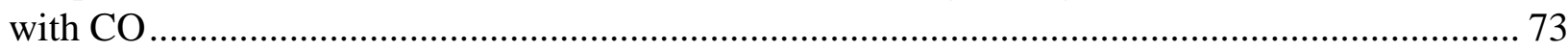

8.1 DSC/TGA during TPR with CO Performance Data Comparison of $\mathrm{CuFeMnO}_{4}$ and Corresponding Single Metal Oxides ...................................................................... 73

8.2 DSC/TGA during TPR with CO Performance Data Comparison of $\mathrm{CuFeMnO}_{4}$ and

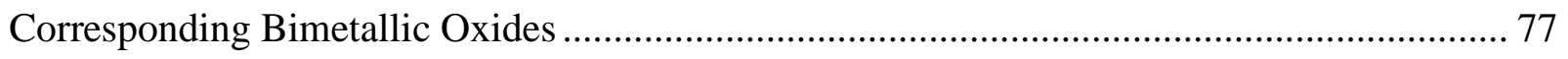

8.3 In-situ XTD TPR with 5\% CO Balance Ar Analysis .............................................. 79

8.4 Isothermal in-situ XRD with Controlled CO Exposures at $800{ }^{\circ} \mathrm{C}$............................. 81

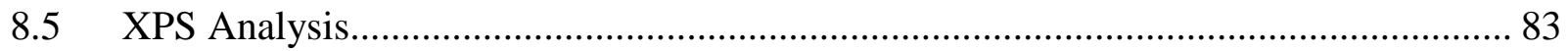

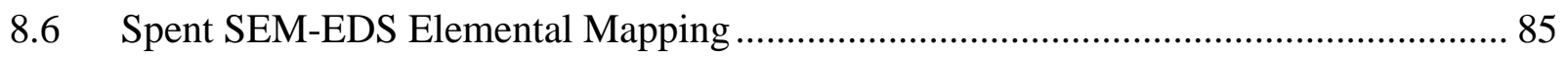

8.7 Isothermal Reaction Model Analysis of $\mathrm{CuFeMnO}_{4}$ and $\mathrm{CO}$.................................. 87 
Chapter 9. Conclusions and Future Recommendations .................................................. 100

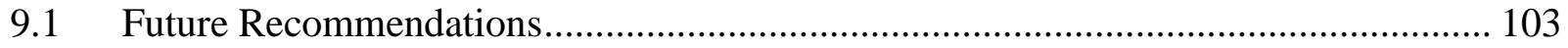

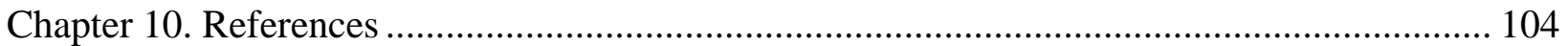




\section{List of Figures}

Figure 2.1 Process flow diagram of dual-reactor CLC system ..................................................... 4

Figure 3.1 Sample pan cross section of DSC/TGA instrumentation ............................................ 16 Figure 5.1 Global rates of reaction comparison of $\mathrm{CuFeMnO}_{4}$ and its (A) bimetallic and (B) single metallic counterparts obtained from 10th TGA reduction cycle at $800^{\circ} \mathrm{C}$........................ 27 Figure 5.2. Global reaction rate comparison of various formulations of the complex trimetallic compositions during the 10th TGA reduction cycle..... 28

Figure 5.3. Isothermal fixed bed flow data during the 10th reduction of $\mathrm{CuFeMnO}_{4}$ with a comparison of the theoretical and experimental mmol of $\mathrm{CO}_{2}$ produced......................................2 29

Figure 5.4. Reaction data of $\mathrm{CuFeMnO}_{4}$ during 10th TGA reduction cycle with $\mathrm{CH}_{4} \ldots \ldots \ldots \ldots \ldots . . . . .30$ Figure 5.5. Cost analysis of metal species and the theoretical cost of manufacturing $\mathrm{CuFeMnO}_{4}$

Figure 5.6. Comparison of attrition index data between an OC which has been tested in a pilotscale reactor and $\mathrm{CuFeMnO}_{4}$

Figure 5.7. Reaction stability of $\mathrm{CuFeMnO}_{4}$ during 10 isothermal fixed bed $\mathrm{CH}_{4}$ reduction/ air oxidation cycles at $800^{\circ} \mathrm{C}$ 34

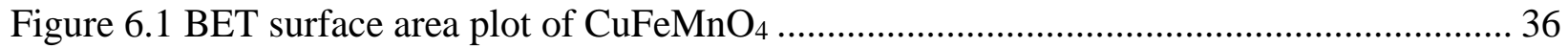

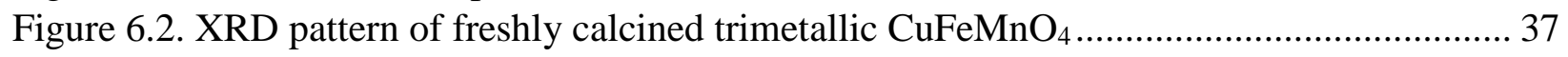

Figure 6.3. DSC/TGA during TPR with $16.6 \% \mathrm{CH}_{4}$ balance Ar performance data comparison of

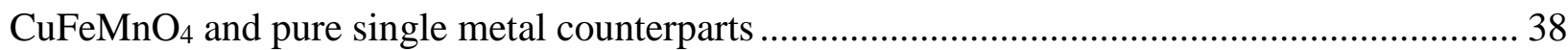
Figure 6.4. DSC/TGA during TPR with $16.6 \% \mathrm{CH}_{4}$ balance Ar performance data comparison of $\mathrm{CuFeMnO}_{4}$ and bimetallic counterparts.

Figure 6.5. In-situ XRD analysis of $\mathrm{CuFeMnO}_{4}$ during temperature programmed reductions with $\mathrm{CH}_{4}$ : (A) $500{ }^{\circ} \mathrm{C}-650{ }^{\circ} \mathrm{C}$ (B) $670{ }^{\circ} \mathrm{C}-850{ }^{\circ} \mathrm{C}$; (1) $\mathrm{CuFeMnO}_{4}$, (2) $\mathrm{MnFe}_{2} \mathrm{O}_{4}$, (3) $\mathrm{MnO}$, (4) $\mathrm{Cu}$,

(5) $\mathrm{Fe}$ 43

Figure 7.1 DSC/TGA during TPR with $20 \% \mathrm{H}_{2}$ balance Ar performance data comparison of $\mathrm{CuFeMnO} 4$ and single metal counterparts $\left(\mathrm{CuO}, \mathrm{Mn}_{2} \mathrm{O}_{3} / \mathrm{Mn}_{3} \mathrm{O}_{4}, \mathrm{Fe}_{2} \mathrm{O}_{3}\right)$... 46

Figure 7.2. DSC/TGA during TPR with $20 \% \mathrm{H}_{2}$ balance Ar performance data comparison of

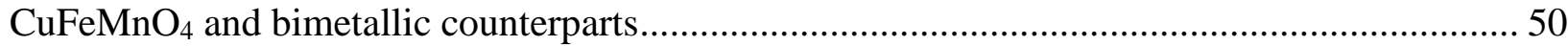

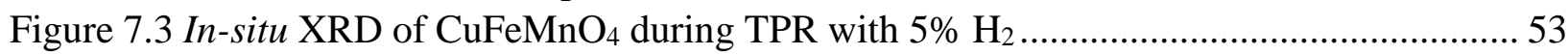

Figure 7.4 In-situ XRD of $\mathrm{CuFeMnO}_{4}$ during TPO with $100 \%$ air............................................ 55

Figure 7.5. Isothermal in-situ XRD data during $\mathrm{H}_{2}$ doses at $800{ }^{\circ} \mathrm{C}$.......................................... 56

Figure 7.6. Fresh $\mathrm{CuFeMnO}_{4} \mathrm{SEM}$-EDS of $\mathrm{Cu}, \mathrm{Fe}$, and $\mathrm{Mn}$ are labeled green, red, and blue respectively (A) SEM exterior imaging (B) SEM cross-section imaging (C) EDS elemental

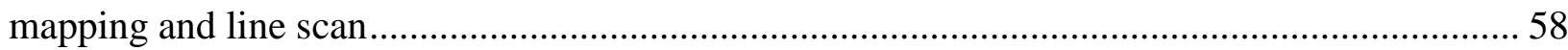

Figure 7.7. Reaction optimization during isothermal $\mathrm{TGA} \mathrm{H}_{2}$ reductions at $800{ }^{\circ} \mathrm{C}$ with respect to varying (A) Concentration (B) Mass (C) Flow rate (D) Particle size........................................ 60 Figure 7.8. (A) TGA reduction of $\sim 25 \mathrm{mg} \mathrm{CuFeMnO} 4$ with $40 \% \mathrm{H}_{2}$ at $800^{\circ} \mathrm{C}$ and (B) Model-free

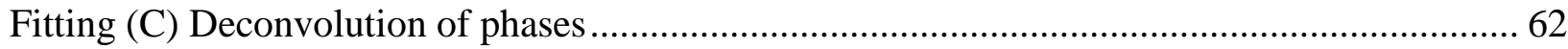
Figure 7.9. Determination of rate constants from (A) 1-D grain boundary and (B) 1-D diffusional

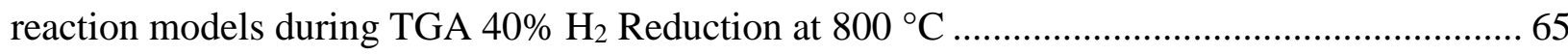
Figure 7.10. Arrhenius curves obtained from reaction modeling ............................................... 70 Figure 7.11. Model prediction with derived reaction parameters ............................................... 71 
Figure 8.1. DSC/TGA during TPR with 20\% CO balance Ar performance data comparison of $\mathrm{CuFeMnO}_{4}$ and corresponding pure metal oxides ....

Figure 8.2. DSC/TGA during with $20 \% \mathrm{CO}$ balance Ar performance data comparison of

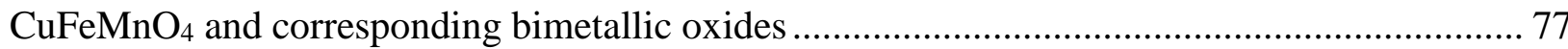

Figure 8.3. In-situ XRD of $\mathrm{CuFeMnO}_{4}$ during TPR with 5\% CO............................................. 79

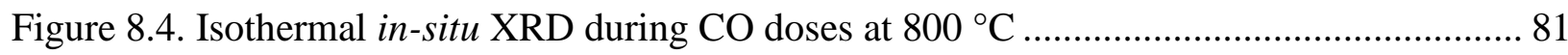

Figure 8.5. XPS analysis of $\mathrm{CuFeMnO}_{4}$ (A) Survey scan (B) Mn 2p (C) Fe 2p (D) Cu 2p........ 83 Figure 8.6. Reacted $\mathrm{CuFeMnO}_{4}$ SEM-EDS of $\mathrm{Cu}, \mathrm{Fe}$, and $\mathrm{Mn}$ are labeled green, red, and blue respectively (A) SEM exterior imaging (B) SEM cross-section imaging (C) EDS elemental mapping and line scan.

Figure 8.7. Reaction optimization during isothermal TGA CO reductions at $800{ }^{\circ} \mathrm{C}$ with respect to varying (A) Concentration (B) Mass (C) Flow rate (D) Particle size....................................... 87 Figure 8.8. (A) TGA reduction of $20 \mathrm{mg} \mathrm{CuFeMnO}_{4}$ with $45 \% \mathrm{CO}$ balance $\mathrm{Ar}$ at $800^{\circ} \mathrm{C}(\mathrm{B})$ Model-free fitting (C) Deconvolution of phases.

Figure 8.9. Determination of rate constants from (A) 1-D grain boundary (B) 1-D diffusional reaction models during TGA $45 \% \mathrm{CO}$ reduction at $800{ }^{\circ} \mathrm{C}$ 92

Figure 8.10. Arrhenius curves from rate constants obtained from reaction modeling ................. 97

Figure 8.11. Nucleation and growth grain boundary model prediction ...................................... 98

Figure 9.1. Particle-scale description of 1-D phase boundary nucleation and growth kinetics.. 102 


\section{List of Tables}

Table 4.1 Summary of possible models and the derived function ........................................................21

Table 5.1 Estimated oxygen transfer capacities for the copper manganese iron oxide system..................31

Table 6.1. Theoretical enthalpy of reaction calculation with pure single metal oxides and $\mathrm{CH}_{4} \ldots \ldots \ldots \ldots . . . .39$

Table 6.2. Experimental and theoretical oxygen transfer capacities of the single metal oxides and $\mathrm{CuFeMnO}$

Table 6.3. Experimental and theoretical oxygen transfer capacities of the bimetallic oxides and $\mathrm{CuFeMnO}$

Table 6.4. Reaction temperatures of the exothermic and endothermic reaction of the various metal oxide

systems

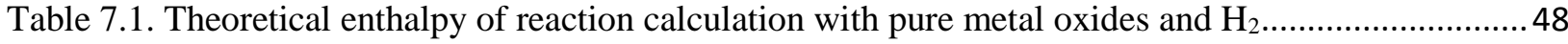

Table 7.2. Experimental and theoretical oxygen transfer capacities of pure metal oxides and $\mathrm{CuFeMnO}_{4} 49$

Table 7.3. Experimental and theoretical oxygen transfer capacities of bimetallic oxides and $\mathrm{CuFeMnO}_{4} 52$

Table 7.4. Reaction constants obtained from 1-D model analysis (A) $\mathrm{CuFeO}_{2}$ (B) $\mathrm{Cu}_{0.5} \mathrm{Mn}_{1.5} \mathrm{FeO}_{4} \ldots \ldots \ldots . .66$

Table 7.5. Definition of dimensionless diffusive and chemical reaction coefficients .............................66

Table 7.6. Dimensionless mass transport analysis of reactor conditions ................................................68

Table 7.7 Dimensionless particle mass transport analysis ...............................................................69

Table 7.8. Intrinsic reaction parameters obtained from Arrhenius curves .............................................69

Table 7.9. Comparison of reaction parameters obtained from the current work and other values found in

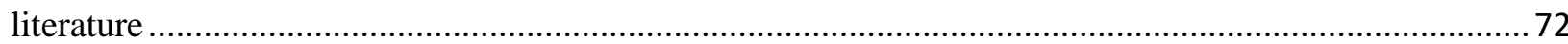

Table 8.1. Theoretical enthalpy of reaction calculation with pure metal oxides and $\mathrm{CO}$........................75

Table 8.2. Experimental and theoretical oxygen transfer capacities of pure metal oxides and $\mathrm{CuFeMnO}_{4} 76$

Table 8.3. Experimental and theoretical oxygen transfer capacities of bimetallic oxides and $\mathrm{CuFeMnO}_{4} 78$

Table 8.4. Reaction Parameters Obtained from Model Analysis and reactor conditions from TGA

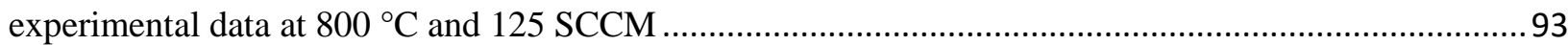

Table 8.5. Dimensionless mass transport analysis of reaction parameters and reactor conditions .............94

Table 8.6. Intrinsic reaction parameters obtained from reaction modeling and Arrhenius curves .............96

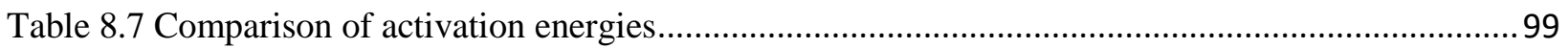


Table of Abbreviations

\begin{tabular}{|c|c|}
\hline$C L C$ & Chemical Looping Combustion \\
\hline$O C$ & Oxygen Carrier \\
\hline$T G A-M S$ & Thermogravimetric Analysis with Mass Spectrometry \\
\hline$F B-M S$ & Fixed Bed reactor with Mass Spectrometry \\
\hline$X R D$ & X-Ray Diffraction \\
\hline$D S C / T G A$ & $\begin{array}{l}\text { Differential Scanning Calorimetry/ Thermogravimetric } \\
\text { Analysis }\end{array}$ \\
\hline$T P R$ & Temperature programmed reduction \\
\hline $\mathrm{CuFeMnO4}$ & Copper Iron Manganese oxide \\
\hline $\mathrm{Sccm}$ & Standard Cubic Centimeters per Minute \\
\hline$U H P$ & Ultra-High Purity \\
\hline $\mathrm{MeO}$ & Metal Oxide \\
\hline$M e$ & Reduced Metal Species \\
\hline $\mathrm{MeO}_{\mathrm{Mw}}$ & Metal Oxide Molecular Weight \\
\hline$M e_{M w}$ & Reduced Metal Species Molecular Weight \\
\hline$M_{t}$ & Instantaneous Mass \\
\hline$M_{i}$ & Initial Mass \\
\hline$M_{f}$ & Final Mass \\
\hline$X$ & Conversion \\
\hline$\varepsilon$ & Porosity \\
\hline$D_{g s}$ & $\begin{array}{l}\text { Diffusive Coefficient between metal oxide solid (s) and } \\
\text { reaction gas }(\mathrm{g})\end{array}$ \\
\hline$C_{g}$ & Reaction Gas Concentration \\
\hline$r$ & Reaction front radius \\
\hline$C_{s}$ & Unreacted Solid Concentration \\
\hline$k_{m}$ & Gas Film Mass Transfer Coefficient \\
\hline$a$ & Stoichiometric Coefficient \\
\hline$m$ & Geometric Solid Reaction Coefficient \\
\hline$C_{s_{i}}$ & Initial Unreacted Solid Concentration \\
\hline
\end{tabular}




\begin{tabular}{|c|c|}
\hline$g(X)$ & Reaction Function \\
\hline$f(x)$ & Reaction Function \\
\hline$g^{-1}(X)$ & Reaction Function Algebraic Rearrangement \\
\hline$b$ & Exponential Coefficient \\
\hline$k_{0}$ & Frequency Factor \\
\hline$E_{a}$ & Activation Energy \\
\hline$R$ & Universal Gas Constant \\
\hline$T$ & Temperature \\
\hline$n$ & Nucleation Coefficient \\
\hline$A$ & Phase $\mathrm{CuFeO}_{2}$ \\
\hline$B$ & Phase $\mathrm{Cu}_{0.5} \mathrm{Mn}_{1.5} \mathrm{FeO}_{4}$ \\
\hline$\varphi$ & Oxygen Density \\
\hline$\rho$ & Oxygen Carrier Density $\left(\mathrm{kg} / \mathrm{m}^{3}\right)$ \\
\hline$R$ & Characteristic Diffusive Length (m) \\
\hline$k_{i}$ & Characteristic Reaction Time $\left(\mathrm{s}^{-1}\right)$ \\
\hline$k_{s}$ & Grain Boundary Surface Reaction Constant $\left(\mathrm{ms}^{-1}\right)$ \\
\hline$d_{i}$ & Characteristic Diffusive Time $\left(\mathrm{s}^{-1}\right)$ \\
\hline$D_{e}$ & Effective Diffusion $\left(\mathrm{m}^{2} \mathrm{~s}^{-1}\right)$ \\
\hline$L$ & Characteristic Crystallite Length (m) \\
\hline$\theta$ & XRD Incident Angle $\left(^{\circ}\right)$ \\
\hline$\beta$ & Structure Angle $\left(^{\circ}\right)$ \\
\hline$k_{d}$ & Deactivation rate constant $(\mathrm{m} / \mathrm{s})$ \\
\hline
\end{tabular}




\section{Chapter 1. Introduction}

The rise in the concentration of $\mathrm{CO}_{2}$ from the combustion of fossil fuels in the atmosphere of the Earth has become an alarming concern in the $21^{\text {st }}$ century [1]. Due to the direct correlation that has been discovered between the accumulation of $\mathrm{CO}_{2}$ in the atmosphere and global temperatures, a world-wide effort has been triggered to curtail the release of the greenhouse gas [1]. This phenomenon is commonly referred to as the "Greenhouse Gas Effect" which is described by the process where heat is trapped by $\mathrm{CO}_{2}$ and prevents the Earth from cooling [1]. In the year 2017, the world produced 32.3 gigatons of $\mathrm{CO}_{2}$ with the production of $\mathrm{CO}_{2}$ demonstrating sustained growth over the past half century [2]. Of this amount of $\mathrm{CO}_{2}$ produced, approximately $60 \%$ of these emissions can be directly attributed to fossil fuel combustion for electricity generation [3]. Clearly, there is no easy solution to this problem due to the heavy dependence on fossil fuels for energy production [4]. Thus, an innovative approach must be employed to curb $\mathrm{CO}_{2}$ emissions from energy generation before the harmful effects of $\mathrm{CO}_{2}$ accumulation become irreversible.

Three categories of $\mathrm{CO}_{2}$ separation processes have been developed to capture $\mathrm{CO}_{2}$ from the combustion of fossil fuels and avoid $\mathrm{CO}_{2}$ emissions. These have been categorized as precombustion, post-combustion, and oxy-fuel combustion and will be discussed briefly [5]. A precombustion approach converts the fossil fuel into an alternate form using water vapor and/or air. During the pre-combustion gasification reaction, the fossil fuel is converted to a mixture of $\mathrm{H}_{2}$ and $\mathrm{CO}$ called synthesis gas, or syngas. The feedstock is further refined with water vapor to form additional $\mathrm{H}_{2}$ and $\mathrm{CO}_{2}$ by means of the water-gas shift reaction. $\mathrm{CO}_{2}$ can then be scrubbed and removed with a commercial solvent before combustion. This process is energy intensive and 
requires additional costs to operate and maintain the pre-treating gasifying and water-gas shift reactors.

Commercialized technology also exists to remove $\mathrm{CO}_{2}$ from the exhaust of power plants in a post-combustion $\mathrm{CO}_{2}$ removal process. In this process, $\mathrm{CO}_{2}$ is absorbed by an amine solvent and removed from the flue gas after combustion of fossil fuels [6]. The amine-based solvent is then pumped to a stripper where the $\mathrm{CO}_{2}$ is removed after the addition of heat and the solvent is recycled back to the flue gas. The heat and pressure requirements to remove $\mathrm{CO}_{2}$ and recycle the solvent are energy intensive which limit efficiency and total power output of this technology [7].

In an oxy-fuel combustion process, purified oxygen, undiluted by nitrogen, is provided for the combustion of fossil fuels. After combustion, $\mathrm{CO}_{2}$ and water vapor are sent to a condenser which provides additional heat, the removal any water vapor, and a purified stream of $\mathrm{CO}_{2}$. In its current status, purified oxygen is provided by an expensive and energy intensive air separation unit. However, oxygen can be delivered to fuel combustion by a metal oxide without the need of an air separation unit in a process called chemical looping combustion (CLC) [8]. This process prevents direct contact of fossil fuels and air, and thus inherently averts nitrous oxide formation and produces sequestration-ready $\mathrm{CO}_{2}$ and heat for power generation. Due to the inherent separation between fuel and air, CLC is the most cost-effective and energy efficient combustion method to meet the energy production requirements while maintaining low $\mathrm{CO}_{2}$ emissions [9]. However, the largest limitation of this $\mathrm{CO}_{2}$ capture technique depends heavily on the selection of a suitable oxygen carrier and a comprehensive study is needed to identify this candidate.

Therefore, this work aims to satisfy the following objectives (1) design a highly reactive and physically robust oxygen carrier, (2) characterize the reactive phase, and (3) develop reaction models that describe the reduction behavior during CLC reactions. The steps in this work to design 
a suitable oxygen carrier for chemical looping combustion should serve as the ideal benchmarks to achieve throughout the process of oxygen carrier development. 


\section{Chapter 2. Review of Literature}

\subsection{Chemical Looping Combustion}

CLC is a promising combustion technology that produces sequestration-ready $\mathrm{CO}_{2}$ [8] [9] [10]. The separation of a pure $\mathrm{CO}_{2}$ stream is achieved by the delivery of oxygen from an oxygen carrier, or metal oxide, for flameless fuel combustion. In addition to heat/power generation, a pure steam of nitrogen-free, $\mathrm{CO}_{2}$ is produced by CLC that advantageously does not require any expensive pre- or post-combustion treatment thus avoiding substantial energy penalties. The cyclical process of fuel combustion and oxygen carrier regeneration is illustrated in Figure 2.1, and the overall reactions that take place in the reducing and oxidizing reactor are shown in Equations 2.1 and 2.2., respectively, where " $\mathrm{MeO}_{4}$ " represents metal oxide and "Me" represents the reduced metal species. Development of oxygen carriers with high performance is critical to the success of CLC operation.

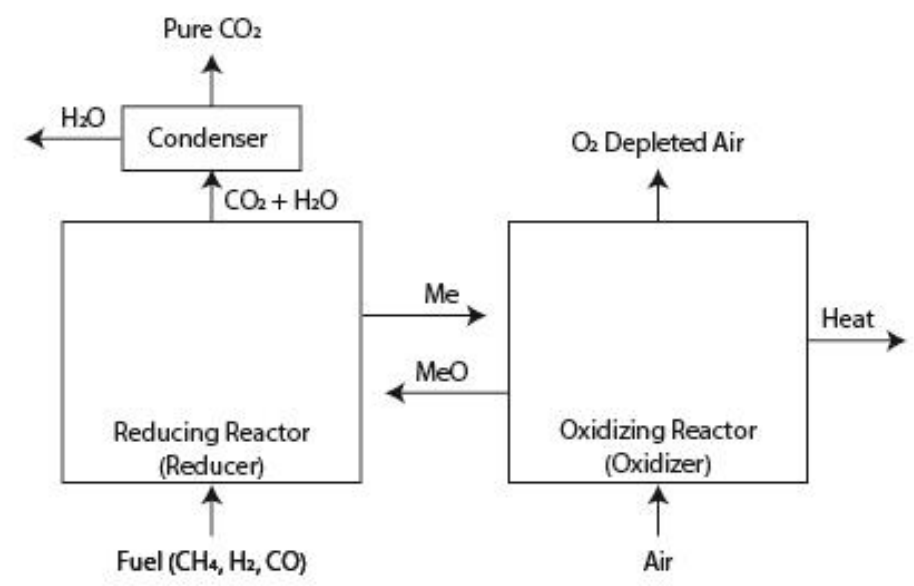

Figure 2.1 Process flow diagram of dual-reactor CLC system

$$
\begin{gathered}
\mathrm{MeO}_{4}+\mathrm{CH}_{4} \rightarrow \mathrm{Me}+\mathrm{CO}_{2}+2 \mathrm{H}_{2} \mathrm{O} \\
\mathrm{Me}+2 \mathrm{O}_{2} \rightarrow \mathrm{MeO}_{4}+\text { Heat }
\end{gathered}
$$


The chemical looping scheme is not limited to $\mathrm{CO}_{2}$ capture and sequestration. The dual reactor system has been proposed for several processes such as chemical looping partial oxidation of $\mathrm{CH}_{4}$, chemical looping gasification of coal, and chemical looping for $\mathrm{H}_{2}$ generation of coal or natural gas [11] [12] [13]. The main advantage in the chemical looping scheme lies in the ability of this system to produce a majority of the heat necessary to complete the desired process in the oxidizing rector with inherent gas-phase separation in the reducing reactor. In circumstances where auto-thermal operation can be achieved, energy input costs would be dramatically decreased due to the inherent ability of the reactor system to produce the necessary heat to complete the desired process.

Pilot-scale operation of CLC with gaseous or gasified solid fuel sources has already demonstrated the feasibility of this technology [14] [15] [16] [17]. Natural mineral ores were used as the carriers for these tests due to their low production cost, but these ores did not possess the most desirable properties required of an oxygen carrier. Thus, synthetic oxygen carrier development has become a new frontier in field of CLC. Synthetic mixed metal oxygen carriers have demonstrated improved performance by achieving deeper levels of reduction and increased reactivity due to well-documented synergistic effects among mixed metal oxide systems compared to natural ores or pure single metal counterparts, however, these synergistic effects are not well understood [18] [19] [20] [21] [22] [23] [24] [25] [26]. Thus, many alkali, alkali-earth, and transition metal oxides have been investigated as potential candidates for synthetic mixed metal oxygen carriers, but the underlying mechanism that promotes performance has yet to be fully comprehended [27] [28] [29] [30]. A comprehensive study is needed to develop a suitable carrier and understand the causes for the improved overall performance of the promising metal oxide 
system. Identifying the reaction pathways of high-performing oxygen carriers is crucial to the development of large-scale CLC reactors.

\subsection{Oxygen Carrier Development}

Strict criteria for oxygen carrier (OC) development has been established to substantiate successful implementation for commercialization [9]. The OC must be highly reactive with the carbonaceous fuel source to prevent unconverted fuel gasses from escaping and contaminating the sequestration-ready $\mathrm{CO}_{2}$. The $\mathrm{OC}$ must have high thermal stability during cycling reactions so that material deterioration can be minimized. The OC must be physically stable with high resistance to attrition if a fluidized bed reactor will be applied. Lastly, the OC and its preparation procedure must be environmentally benign and inexpensive to accommodate the high solids-inventory throughput demanded by CLC. As a result of the demanding criteria, CLC is still searching for the ideal OC, and results have shown that copper iron manganese oxide $\left(\mathrm{CuFeMnO}_{4}\right)$ has the potential to be this candidate [31]. During isothermal analysis, $\mathrm{CuFeMnO}_{4}$, synthesized from the sol-gel method of metal nitrate salts, demonstrated very high reactivity [21]. However, the details on the formation of the trimetallic phase and its enhanced reactivity are not fully understood. Thus, it is necessary for material design and CLC reactor optimization to characterize, model, and analyze the reaction pathway of the complex trimetallic OC derived from economically and environmentally friendly precursor metal oxides. The influence of these single metal components will be described next.

Copper oxide has a distinct advantage as an OC due to its documented exothermic reduction reaction with $\mathrm{CH}_{4}$ [27]. It has been studied extensively as a component in many mixed metal oxygen carriers due to its high oxygen transfer capacity, or oxygen utilization, and has demonstrated improved performance and reactivity when incorporated with another metal oxide 
[27] [32] [33] [34]. It has also been highlighted for use in oxygen uncoupling processes where oxygen is released spontaneously from the metal oxide lattice at reaction temperatures. However, the use of a support is required due to agglomeration issues caused by the low melting point of copper oxide [35]. The exothermic nature of the reaction with $\mathrm{CH}_{4}$ distinguishes copper oxide from other metal oxides candidates.

Iron oxide has been widely studied as an $\mathrm{OC}$ due to its low cost, fair performance with synthesis gas, and high reactivity during oxidation [36] [37] [38] [39] [40] [41] [42] [43] [44] [45]. However, in $\mathrm{CH}_{4}$, iron oxide exhibits low reactivity during reduction and is commonly incorporated with other metal oxides to improve performance [39]. The formation of these mixed metal-ferrite phases influences OC performance depending on the spinel or perovskite formed [44]. The performance of iron oxide is influenced by solid-solid phase transitions as well. The inexpensive and environmentally benign features of iron oxide highlight the several components that are desirable in synthetic OC design.

Manganese oxide has also been studied due to its reasonable reactivity with $\mathrm{CH}_{4}$ and oxygen uncoupling properties [45] [46] [47] [48] [49] [50]. Improved performance and reaction stability have been reported for manganese oxides incorporated with additional metal oxides. Like iron oxide, solid-solid phase transitions influence the reaction pathway of manganese oxide and various complex metal oxide phases can be formed with incorporation of additional metal oxides. However, the oxidation of $\mathrm{MnO}$ to the original oxidation state is difficult [50]. Overall, incorporation of manganese oxide is an economical and environmentally benign option to improve stability and oxygen transfer capacity in synthetic oxygen carriers.

Manganese oxide in particular is the least studied of the three components. The reduction of $\mathrm{Mn}_{2} \mathrm{O}_{3}$ to metallic $\mathrm{Mn}$ yields a total oxygen transfer capacity of approximately $31 \%$. Despite 
the large amount of oxygen available for CLC reactions, much of this is unobtainable. The reduction of $\mathrm{Mn}_{2} \mathrm{O}_{3}$ to $\mathrm{MnO}$ with $\mathrm{CH}_{4}$ is favorable at temperatures above $450{ }^{\circ} \mathrm{C}$, however the regeneration of $\mathrm{MnO}$ to $\mathrm{Mn}_{2} \mathrm{O}_{3}$ is thermodynamically limited at temperatures exceeding $800{ }^{\circ} \mathrm{C}$ [51]. Thus, in a typical CLC reactor operating between $800^{\circ} \mathrm{C}-1000^{\circ} \mathrm{C}$ it is thermodynamically impossible regenerate the higher oxidative form of manganese oxide. The manganese oxide system can generate an oxide in the $4^{+}$valance state in the form of $\mathrm{MnO}_{2}$. However, this compound is not stable at temperatures exceeding $500{ }^{\circ} \mathrm{C}$ due to a thermal decomposition reaction that releases oxygen and yields $\mathrm{Mn}_{2} \mathrm{O}_{3}$ [52]. The reduction of $\mathrm{MnO}$ to $\mathrm{Mn}$ is also thermodynamically limited in typical operating conditions of $\mathrm{CLC}$ for $\mathrm{H}_{2}, \mathrm{CO}$, and $\mathrm{CH}_{4}$ unlike the reduction of iron oxide which has demonstrated the ability to form metallic Fe with $\mathrm{H}_{2}$ or $\mathrm{CO}$ [27].

Despite restrictions on operating conditions for manganese oxides in CLC, it is speculated that the limitation of $\mathrm{MnO}$ to form metallic $\mathrm{Mn}$ during reduction provides reaction stability properties by acting as a metal oxide support [53]. In addition, the reduction of metal oxides is typically endothermic with $\mathrm{CH}_{4}$, however, the reduction of $\mathrm{Mn}_{2} \mathrm{O}_{3}$ to $\mathrm{Mn}_{3} \mathrm{O}_{4}$ is exothermic although, as mentioned, the regeneration of $\mathrm{Mn}_{2} \mathrm{O}_{3}$ under CLC conditions is not favorable [27]. Exothermic reactions during reduction and inherent reaction stability properties are highly soughtafter features that could be achievable in complex multi-components oxygen carriers that contain manganese oxides.

Although the single metal oxides contain desirable properties, each of the metal oxide systems suffer from significant setbacks. Thus, interest in multi-component systems to promote the desirable properties has risen. As previously mentioned, performance enhancement has been documented during CLC reactions with $\mathrm{CuFeMnO}_{4}$ synthesis from metal nitrate salts. However, carriers synthesized from nitrate salts are not practical in CLC due to $\mathrm{NO}_{\mathrm{x}}$ formation during 
synthesis and metal oxides must be used as precursors. A clear understanding of the formation of the highly reactive phase synthesized from metal oxide precursors and the influence of this phase on reactivity is still unknown. Due to the complexity of trimetallic $\mathrm{CuFeMnO}_{4}$ oxide, traditional characterization methods such as X-ray diffraction (XRD) cannot conclusively identify dominant reactive phase and further analysis is required to characterize the unique phase especially when metal oxide precursors are used during synthesis. After the phase has been characterized, reaction modeling can be conducted to identify the mechanisms that are contributing to the exceptional performance observed during CLC reactions. Reaction modeling of complex systems can contain a number a variables and clear elucidation of the phase and the types of reaction mechanisms that could occur during CLC are vital to providing realistic reaction models and understanding observed reaction phenomena.

\subsection{Reaction Mechanisms}

In order to gain a deeper understanding of the synergistic effects of the trimetallic system and consequently the improved performance, a comprehensive analysis must be conducted to model the reaction mechanisms that are occurring during CLC operation. There exists a number of possible gas/solid reaction mechanisms that can influence the behavior of the reaction between a particle and reaction gas. Using a conventional solid-state and model-free approaches, experimental thermogravimetric analysis (TGA) reaction data is analyzed with established mathematical models to calculate reaction parameters. The validity of the models is then tested with spectroscopic techniques.

Commonly, the shrinking core model is used to describe the behavior of the interaction of a solid during reaction with a gas. A number of models have been proposed and their deviations from the shrinking core model will be discussed briefly. A common model chosen to describe a 
reaction between a metal oxide and a reducing gas is the unreacted shrinking core model proposed by Ishda, M. [54] [55]. In this description, the gaseous molecule reacts with the solid particle leaving behind a product shell. This reaction can be dominated by a number of different phenomena and the assumption chosen to define the phenomena controlling the reaction will be reflected in the deviation of the model and experimental data. Ishda, et al. have described three common types of conditions which are classified as chemical, diffusional, and mass transfercontrolled reactions. In chemically controlled environments, there is no limitation of the reaction gas diffusing through the reacted product shell. In diffusion-controlled environments, the reaction between the grains and the reaction gas is limited by the rate at which the reaction gas can reach the unreacted core, and in mass transport-controlled reactions the external film of gases surrounding the reacting solid particle controls the rate of reaction.

Beyond grain phenomena, the particle can undergo physical and chemical changes as well. Park, et al. proposed the crackling core model to describe flakey product shells produced after reaction [56]. Pigford, et al. and Sohn, et al. have suggested changes to particle porosity to control reaction mechanisms [57] [58]. Rammachandram, et al. have described reaction conditions using a single pore model [59]. In addition to morphological changes, chemical changes can also occur. In a series of papers, Avrami presented the theory and application of the nucleation and growth model to describe solid-solid phase transitions during reactions [60] [61] [62].

These fundamental equations were designed with coal particles envisioned but still provide the fundamental basis for the solid-state particle-scale modeling with reactions involving oxygen carriers and fossil fuels. However, due to differences in reactivity experienced between coal/ oxygen and oxygen carriers/reducing gases, there lacks consistency in the literature with 
regard to the exact nature of the mechanisms taking place during CLC reactions. Zafar, et al. proposed a linear reaction mechanism which would suggest mass transfer through the film as the rate determining step during modeling and once gain diffusional effects cannot be totally neglected [63]. Hossain, et al. conducted model analysis with temperature programmed reductions and nucleation growth kinetics [64]. The analysis performed in this work determined that the activation energies provided were controlled by internal mass transfer limitations, yet no discussion of the Thiele modulus, effectiveness factor, or Weisz-Prater parameter had been conducted to validate such a claim. The unique reactivity observed from bulk oxygen donation has provided a number of problems in reaction modeling found in literature to date and a systematic kinetic evaluation is needed to determine intrinsic rate constants of these particles for large-scale reactor design.

Another issue that can occur during kinetic modeling of OC's with carbonaceous fuels is carbon formation from the catalytic deposition of carbon from $\mathrm{CH}_{4}$ decomposition, or from $\mathrm{CO}$ during the Boudouard reaction. During reduction reactions with $\mathrm{CH}_{4}$ and $\mathrm{CO}$, carbon can deposit on exposed catalytic sites to form surface carbon as well as $\mathrm{H}_{2}$ and $\mathrm{CO}_{2}$, respectively [65] [66]. This behavior is typically observed by weight gain when using a thermogravimetric analyzer (TGA) as well as a simultaneous product shift. It is speculated that the deactivation kinetics that occur during reduction with $\mathrm{CH}_{4}$ and $\mathrm{CO}$ can be represented utilizing similar mathematical principles.

After an establish model has demonstrated the best fit with experimental data, a series of spectroscopic techniques must be employed to confirm the validity of the model. Given the complexity of the trimetallic system, X-ray diffraction (XRD) analysis, although helpful, is not sufficient enough to identify changes to the chemical composition and describe reaction 
mechanics. To complement the data experimental reaction data, XRD, X-ray photoelectron spectroscopy (XPS), Brunauer-Emmett-Teller (BET) surface area analysis, scanning electron microscopy (SEM), and simultaneous differential scanning calorimetry/ thermogravimetric analysis (DSC/TGA) must be utilized to characterize changes to the metal cation oxidative state, and the physical changes to the pellet described by the kinetic phenomena.

A systematic approach to the design of a high performing OC must be conducted to substantiate the use of the carrier for chemical looping combustion. Once a viable candidate has been designed and tested a structural and kinetic elucidation of the unique OC must be conducted to understand and describe the observed synergistic effects of the high performing material A robust understanding of the reaction pathway of this novel OC would bridge the gap between fundamental reaction phenomena and process design which is still highly uncertain to date and lacks consistency in literature. A comprehensive description of CLC reactions and mechanisms is necessary for future OC design and the improving large-scale reactor design.

Thus, the objective of this work is as follows: (1) Design a high performing oxygen carrier suitable to perform chemical looping combustion (2) Identify the reactive phase of the oxygen carrier candidate (3) Develop a comprehensive mechanistic understanding of the reduction reaction to provide mathematical models for process scale-up and simulation. 


\section{Chapter 3. Experimental}

\subsection{OC Pellet Preparation}

$\mathrm{MnO}_{2}(\sim 44 \mu \mathrm{m})$, from Alfa Aesar, $\mathrm{CuO}(<10 \mu \mathrm{m})$, from Sigma Aldrich, and commercially available iron ore Amiron $40 \mathrm{H}(<40 \mu \mathrm{m})$, from Applied Minerals, were used to synthesize the oxygen carriers. Stoichiometric amounts the metal oxide powders to form single, bi-, and trimetallic oxygen carriers, as indicated by subscripts in the chemical formulas, were evenly mixed in a cylindrical rotary mixer. Deionized water was added dropwise while mixing the metal oxide precursors until pellets in the desired size range were obtained. These pellets were heated in a furnace at $2{ }^{\circ} \mathrm{C} / \mathrm{min}$ to $1000{ }^{\circ} \mathrm{C}$ and kept isothermally for six hours except for pure $\mathrm{CuO}$ which was calcined at $900{ }^{\circ} \mathrm{C}$ for six hours. All samples were calcined in air.

3.2. Reduction/ Oxidation TGA with Online Effluent Gas Analysis by Mass Spectrometry (TGA-MS)

Experiments were conducted on a Thermal Analysis Systems Q50 TGA and the effluent product stream was continuously monitored by a Pfeiffer Vacuum Omnistar Mass Spectrometer. A specified amount $(15-60 \mathrm{mg})$ of OC with a particle diameter range of 40-60 mesh $(425-250 \mu \mathrm{m})$ was placed on a platinum pan, from TA instruments, during the multi-cycle reduction/oxidation testing. As the multi-cycle reduction/oxidation experiment progressed, the weight change of the sample was monitored, and the product gas composition was measured using the mass spectrometer. The TGA performance was optimized by varying flow rate and particle size to limit any diffusional mass transfer effects.

3.3. Micromeritics Fixed Bed Reactor with Online Effluent Gas Analysis by Mass Spectrometry (FB-MS)

Fixed bed flow reactor experiments were conducted in an Atmospheric (1 atm) Autochem Micromeritics 2910 reactor with online product effluent monitored by means of a Pfeiffer Vacuum 
Omnistar Mass Spectrometer. Prior to sample loading, a small amount of quartz wool was placed at the base of the sample tube. Approximately one gram of material was charged to the sample tube.

\subsection{Attrition Index}

ASTM 5757-11 method was used to evaluate attrition. The sample was subjected to sustained high flows of $\sim 300$ - $400 \mathrm{~m} / \mathrm{s}$ in a jet-cup Attri-AS Material Technology Unit and fines from deteriorated particles were collected in a filter bank after 1, 3 and 5 hours of exposure. The data obtained is then converted to attrition index, which is the calculation of fines collected as a percentage of the material's total mass over time.

\subsection{X-ray Diffraction (XRD)}

XRD patterns were taken using a PANalytical X'Pert Pro instrument and analyzed with High Score Plus software. Scans were taken from 5-100 29, current was set at $40 \mathrm{~mA}$, and the voltage of the wavelength generator was set at $42 \mathrm{mV}$. Oxygen carriers were crushed and sieved $(<74 \mu \mathrm{m})$ prior to placement on the sample stage. Reference patterns 04-007-8976, 00-041-0254, 00-013-0162, and 00-020-0358 were used for $\mathrm{Fe} 3 \mathrm{O} 4, \mathrm{CuO}, \mathrm{Mn3O} 4$, and $\mathrm{CuFeMnO}_{4}$ respectively for analysis in High Score Plus.

In-situ XRD temperature programed reductions were performed in an Anton Par Hot Stage. Approximately $160 \mathrm{mg}$ of OC was crushed and sieved to obtain a particle size range $<74 \mu \mathrm{m}$. This amount will ensure complete coverage of the sample stage during XRD analysis. The sample was heated at $3{ }^{\circ} \mathrm{C} / \mathrm{min}$ from ambient to $900{ }^{\circ} \mathrm{C}$ with the desired reaction gas. Reference patterns 04002-0625, 04-008-6796, 04-002-1833, 04-003-2430, 04-002-8196, 04-002-8160, and 04-002- 
8196 were used to identify $\mathrm{MnFe}_{2} \mathrm{O}_{4}, \mathrm{MnO}, \mathrm{Fe}, \mathrm{Cu}, \mathrm{CuFeO}_{2}, \mathrm{Fe}_{0.5} \mathrm{Mn}_{0.5} \mathrm{O}$, and $\mathrm{Cu}_{0.5} \mathrm{Mn}_{1.5} \mathrm{FeO}_{4}$ respectively in High Score Plus.

Isothermal in-situ XRD analysis was conducted at $800{ }^{\circ} \mathrm{C}$. The sample was heated in air to the desired temperature and allowed to purge with $\mathrm{N}_{2}$. Then, the sample was exposed to $5 \mathrm{~mL}$ incremental injections of the reaction gas, $\mathrm{H}_{2}$ or $\mathrm{CO}$.

3.6. Simultaneous Differential Scanning Calorimetry and Thermogravimetric Analysis during Temperature Programmed Reduction (DSC/TGA TPR)

Simultaneous DSC/TGA TPR with $\mathrm{CH}_{4}$ was conducted in a Thermal Analysis System Q 600 SDT. Simultaneous DSC/TGA measures deviations in weight and heat flow between a sample beam and reference beam inside a furnace. Prior to the temperature ramps, the signals from the sample and reference beams were allowed to stabilize, and the instrument was tared. Then, approximately $15 \mathrm{mg}$ of $\mathrm{OC}$ was loaded into the sample pan. The $\mathrm{OC}$ was crushed and sieved to obtain a particle size range $<74 \mu \mathrm{m}$ before loading, and the base of the sapphire pan was covered evenly with a thin layer of sample to prevent any diffusional resistance of the reaction gas through OC layers. This experimental setup ensures that the heat flow was only from reactions taking place at the interface of the reaction gas and the sample particles as shown in the sample pan cross section in Figure 3.1. The sample was heated at $20^{\circ} \mathrm{C} / \mathrm{min}$ from ambient to $1000^{\circ} \mathrm{C}$ in the desired reaction atmosphere. After initial calibration with respect to the reference pan, only the sample pan was removed to be cleaned between experiments. 

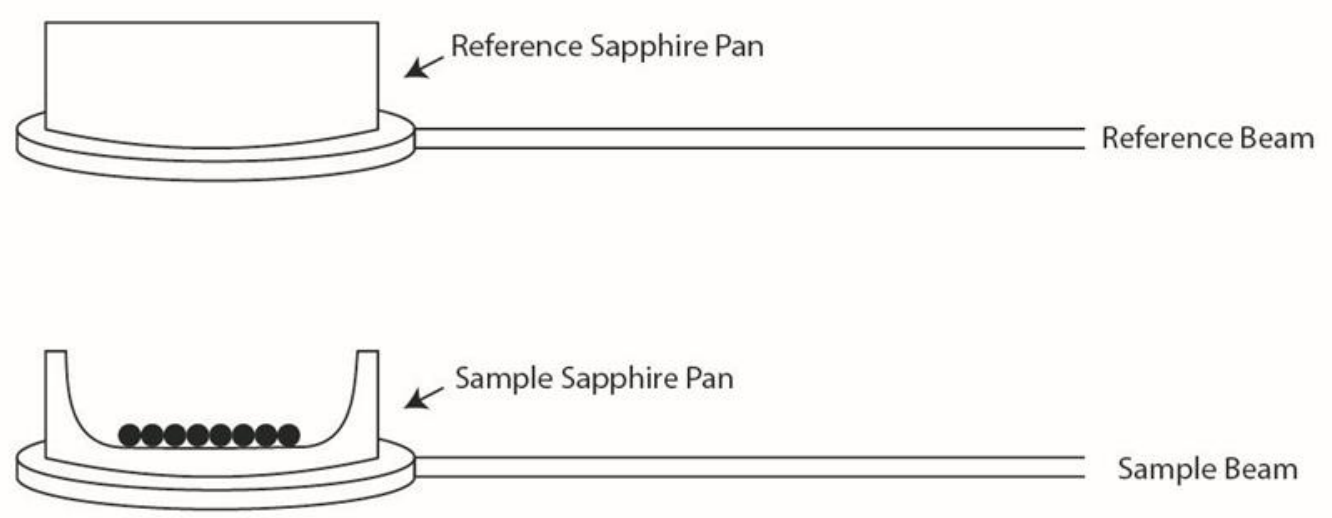

Figure 3.1 Sample pan cross section of DSC/TGA instrumentation

Heat flow and weight change in the simultaneous DSC/TGA were recorded as the difference in signals between the sample beam and the reference beam. It should be noted that DSC/TGA exothermic heat flow is represented by signals in the positive y-axis and endothermic heat flow is represented in the negative $\mathrm{y}$-axis over the temperature range shown on the $\mathrm{x}$-axis.

\subsection{Brunauer-Emmett-Teller (BET) Analysis}

BET analysis was conducted in a Micromeritics ASAP 2020 Surface Area Pore Analyzer with an assumed $\mathrm{N}_{2}$ cross section of $0.16 \mathrm{~nm}^{2}$ and internally calculated void space to account for the use of a filler rod.

\subsection{X-Ray Photoelectron Spectroscopy (XPS)}

The XPS consisted of a Physical Electronics (PHI) model 548 equipped with a Omni-III spherical capacitance analyzer, a model 04-548 X-ray source, a 50-096 X-ray Source ControlSupply, a 04-303 differentially pumped ion gun on the analysis chamber and a 04-191 ion gun on the sample preparation chamber. The analysis chamber routinely operated within a pressure range of $10^{-8}$ to $10^{-9}$ Torr $\left(1.3 \times 10^{-6}\right.$ to $1.3 \times 10^{-7} \mathrm{~Pa} ; 1$ Torr $\left.=1.322368 \mathrm{~Pa}\right)$. The system was calibrated in 
accordance with PHI procedures [67] with photoemission lines: $\mathrm{E}_{\mathrm{b}} \mathrm{Cu} 2 \mathrm{p}_{3 / 2}=932.7 \mathrm{eV}, \mathrm{E}_{\mathrm{b}} \mathrm{Au}$ $5 f_{7 / 2}=84.0 \mathrm{eV}$, and $\mathrm{E}_{\mathrm{b}} \mathrm{Ag} 5 \mathrm{~d}_{5 / 2}=368.3 \mathrm{eV}$. The binding energies were referenced to $\mathrm{C}_{1 \mathrm{~s}}=284.8$ $\mathrm{eV}$ for adventitious carbon. Acquisition and initial analyses was carried out utilizing Auger-scan software by RBD Instruments; additional analyses were carried out utilizing CASA-XPS. Regions of interest were acquired with a pass energy (PE) of $11.75 \mathrm{eV}$ at $15 \mathrm{kV}$ and $22 \mathrm{ma}$ (350 Watts); survey spectra were acquired under similar conditions at $\mathrm{PE}=59.35$.

\subsection{Scanning Electron Microscopy with Energy Dispersive Spectroscopy (SEM-EDS)}

The X-ray Microanalysis system consisted of a JEOL-840A SEM interfaced to a Noran NS-6 X-ray microanalysis system. The JEOL-840A is equipped with an annular backscatter detector (BSED), an ET type secondary electron detector (SED), and a Noran Ultra-Dry Energy Dispersive Spectrometer (EDS); EDS detector was calibrated utilizing the $\mathrm{Cu} \mathrm{K} \alpha=8.041 \mathrm{kV}$ [68]. The JEOL 840A SEM was routinely operated in a pressure range of $10^{-6}-10^{-5}$ Torr $\left(1.3 \times 10^{-5}\right.$ to $1.3 \times 10^{-6} \mathrm{~Pa} ; 1$ Torr $\left.\approx 1.3224 \mathrm{~Pa}\right)$.

\subsection{Data Evaluation}

Experimental oxygen transfer capacity was calculated by taking the difference of the initial mass, $M_{i}$ and the instantaneous weight loss, $M_{t}$, and dividing the difference by the initial mass as shown in Equation 3.1. It was assumed that the weight loss was attributed to oxygen leaving the metal oxide lattice and reacting with $\mathrm{CH}_{4}$ to form the gaseous by-products in Equation 2.1.

$$
\text { Experimental Oxygen Transfer Capacity (\%) }=100 \% * \frac{M_{i}-M_{t}}{M_{i}}
$$

Theoretical oxygen transfer capacity was calculated by assuming the initial metal oxide $(\mathrm{MeO})$ and was fully reduced to metal species $(\mathrm{Me})$ in Equation 2.1. The molecular weights of 
these species, $\mathrm{MeO}_{\mathrm{Mw}}$ and $\mathrm{Me}_{\mathrm{Mw}}$, were used to calculate the theoretical oxygen transfer capacity as shown in Equation 3.2. It was assumed that weight loss was attributed to oxygen leaving the metal oxide lattice in the form of the gaseous by-products in Equation 2.1.

$$
\text { Theoretical Oxygen Transfer Capacity }(\%)=100 \% *\left(1-\frac{M e_{M w}}{M e O_{M w}}\right)
$$

\subsection{FactSage Calculations}

Theoretical thermochemical and reaction data were calculated with FactSage 7.0 software to obtain enthalpic heat flow data during reactions with $\mathrm{CH}_{4}, \mathrm{H}_{2}$, and $\mathrm{CO}$ with $\mathrm{CuO}, \mathrm{Fe}_{2} \mathrm{O}_{3}$ and $\mathrm{Mn}_{2} \mathrm{O}_{3}$. These theoretically calculated heat flow values were compared with observed heat flow data during DSC/TGA TPR experiments. 


\section{Chapter 4. Reaction Modeling}

\subsection{Conversion}

Experimental TGA weight change data was compared with accepted solid-state reaction models to evaluate goodness of fit. Experimental conversion, $X(t)$, during TGA experiments was calculated with Equation 4.1 where $M_{i}$ represented the mass of the sample before reduction, $M_{f}$ represented the mass upon completion of the reaction, and $M_{t}$ represents the instantaneous mass at any point during the reaction.

$$
\frac{M_{i}-M t}{M_{i}-M_{f}}=X(t)
$$

\subsection{Chemical and Diffusion Reaction Controls}

The unreacted-core shrinking model derived by Ishda, et al. is well-known and has been used as the basis for gas/solid reactions models extensively in literature [54] [55]. The unreacted core models are derived from the material balance in Equation 4.2 with the assumed boundary conditions in Equations $4.3-4.5$ where $\varepsilon, \mathrm{C}_{\mathrm{g}}, \mathrm{D}_{\mathrm{gs}}, \mathrm{r}, \mathrm{a}, \mathrm{C}_{\mathrm{s}}$ represent porosity, gaseous concentration, gas/solid diffusion, reaction radius, stoichiometric coefficient, and concentration of oxygen in the solid respectively. Dictated by the boundary conditions, the reaction is controlled by external gas film diffusion surrounding the particle, $\mathrm{k}_{\mathrm{m}}$ is the external mass transfer coefficient in Equation 4.3, the gradient of that concentration at the core of the particle in Equation 4.4, and the initial unreacted material concentration in Equation 4.5.

$$
\begin{gathered}
\varepsilon\left(\frac{\partial C_{g}}{\partial t}\right)=D_{g s}\left[\frac{1}{r^{2}} \frac{\partial}{\partial r}\left(r^{2} \frac{\partial C_{g}}{\partial r}\right)\right]+a \frac{\partial C_{s}}{\partial t} \\
r=1 \text { and } C_{g}=k_{m}\left(C_{g_{i}}-C_{g}\right) \\
r=0 \text { and } \frac{\partial C_{g}}{\partial r}=0
\end{gathered}
$$




$$
C_{s}=C_{s_{i}} \text { and } t=0
$$

For particles, such as metal oxides, the assumptions by Ishida, et al. are used as the basis to describe the reaction phenomena. It is assumed that three special cases exist where either (1) the chemical reaction taking place at the interface of the grain boundary is the rate determining step, where $k<D$. (2) Diffusion of the gaseous intermediates in and out of the ash-product layer, where $\mathrm{D}<\mathrm{k}$, controls the rate of the reaction. And (3), where external gas film mass transfer surrounding the particle control the reaction. Mass transfer phenomenon is described by zeroth ordered kinetics shown in Equation 4.3.

In case 1, it is assumed that the concentration of the reaction gas is constant between the reaction zone and the bulk gas stream and thus no reaction gas concentration gradient exists within the particle and Equation 4.2 simplifies to Equation 4.6. It is assumed in Equation 4.6 that the consumption of reaction gas is equal to the consumption of oxygen in the OC. After substituting Equation 4.7, Equation 4.8, and Equation 4.9 in Equation 4.6 to relate conversion, $\mathrm{X}$, and the reaction front, the chemical reaction controlling rate equation is formed in Equation 4.10 and the differentiation of this equation can be found in Error! Reference source not found. for 2-D or 3D contraction depending on the appropriate value obtained for the geometric coefficient, "m." 
Table 4.1 Summary of possible models and the derived function

\begin{tabular}{|c|c|c|c|}
\hline Model & $f(X)=\left(\frac{1}{k}\right) \frac{d X}{d \tau}$ & $g(X)=k \tau$ & $X=g^{-1}(\tau)$ \\
\hline JMAEK & $n(1-X)[-\ln (1-X)]^{\frac{n+1}{n}}$ & {$[-\ln (1-X)]^{n \frac{1}{n}}$} & $1-\exp \left[-(k \tau)^{n}\right]$ \\
\hline Prout-Tompkins & $X(1-X)$ & $\ln \left[\frac{X}{1-X}\right]$ & {$[1+1 / \exp (k \tau)]^{-1}$} \\
\hline 1-D Contraction & 1 & $X$ & $k t$ \\
\hline 2-D Contraction & $2(1-X)^{\frac{1}{2}}$ & $1-(1-X)^{\frac{1}{2}}$ & $1-(1-k \tau)^{2}$ \\
\hline 3-D Contraction & $3(1-X)^{\frac{2}{3}}$ & $1-(1-X)^{\frac{1}{3}}$ & $1-(1-k \tau)^{3}$ \\
\hline 1-D Diffusion & $\frac{1}{2} X$ & $X^{2}$ & $(k \tau)^{\frac{1}{2}}$ \\
\hline 3-D Diffusion & $\frac{3}{2}(1-X)^{\frac{2}{3}}\left[1-(1-X)^{\frac{1}{3}}\right]$ & $\left(1-(1-X)^{\frac{1}{3}}\right)^{2}$ & $1-\left[1-(k \tau)^{\frac{1}{2}}\right]^{3}$ \\
\hline Zeroth Order & 1 & $X$ & $k \tau$ \\
\hline First Order & $1-X$ & $-\ln (1-X)$ & $1-\exp (-k \tau)$ \\
\hline Second Order & $(1-X)^{2}$ & {$\left[\frac{1}{(1-X)}\right]-1$} & $1-(k \tau+1)^{-1}$ \\
\hline Third Order & $(1-X)^{3}$ & {$\left[\frac{1}{(1-X)^{2}}\right]-1$} & $1-(k \tau+1)^{\frac{-1}{2}}$ \\
\hline $\mathrm{z}^{\text {th }}$ Order & $(1-X)^{Z}$ & {$\left[\frac{1}{(1-X)^{z-1}}\right]-1$} & $1-(k \tau+1)^{\frac{-1}{z-1}}$ \\
\hline Power Law & $X^{-1 / e}$ & $\frac{X^{-\frac{1}{e}+1}}{\frac{-1}{e}+1}$ & $k \tau^{b}$ \\
\hline
\end{tabular}




$$
\begin{gathered}
a \frac{\partial C_{s}}{\partial t}=a C_{s}^{m} C_{g_{i}} \\
C_{s}=C_{S_{i}}(1-X) \\
\frac{\partial C_{s}}{C_{s_{i}} \partial t}=\frac{\partial X}{\partial t} \\
X=\left(1-\frac{4 / 3 \pi r^{3}}{4 / 3 \pi R^{3}}\right) \\
-\frac{\partial X}{\partial t}=k(1-X)^{\frac{1}{m}} C_{g_{i}} C_{S_{i}}
\end{gathered}
$$

In case 2, diffusion of the reaction gas through the product layer of the reacted shell of the particle controls the rate of reaction by Fick's second law of diffusion in Equation 4.11 is rearranged in Equation 4.12 and then differentiated to form Equation 4.13. In this step, it is assumed that the difference of the concentration of the reaction gas at the shell and reaction front is negligible. It is also assumed Equation 4.13 that the rate of consumption of the reaction gas is equal to the rate of consumption of oxygen.

$$
\begin{gathered}
\left(\frac{\partial C_{g}}{\partial t}\right)=D_{g s}\left[4 \pi r^{2}\left(\frac{\partial C_{g}}{\partial r}\right)\right]=\text { constant } \\
\int\left(\frac{\partial C_{g}}{\partial t}\right) \frac{1}{r^{2}} d r=4 D_{g s} \pi d C_{g} \\
\frac{\partial C_{g}}{\partial t}=\frac{4 D_{g s} \pi d C_{g}}{\frac{1}{r}-\frac{1}{R}}=\frac{\partial C_{s}}{\partial t} \\
\frac{\partial C_{s}}{\partial t}=-4 \rho \pi r^{2} \frac{\partial r}{\partial t}
\end{gathered}
$$

Where the rate of consumption of oxygen is described by Equation 4.14. 


$$
\frac{t}{\tau}=1-3(1-X)^{\frac{2}{3}}+2(1-X)
$$

Upon combination, integration, and rearrangement of Equation 4.13 and 4.14 the diffusive controlled rate equation is derived in Equation 4.15 where reaction radius has been substituted for conversion as shown previously in Equation 4.9. Total diffusive control is not a common rate control mechanism, but has been reported in the reduction of iron oxide pellets greater than $7 \mathrm{~mm}$ which is far beyond the reasonable particle size range for CLC [69]. In addition, this model does not form a numerical solution for model-fitting. However, the combination of mixed chemical and diffusion reaction control is a common occurrence in the reaction of metal oxides and is formed by the combination of Equation 4.16, and the 3-D chemical contraction model to form the D-3 diffusion model shown in Table 4.1.

$$
\frac{\partial C_{g}}{\partial t}=D_{s}^{O^{2-}} \frac{\partial C_{s}}{\partial r}
$$

\subsection{Nucleation and Growth}

To describe the phenomenon of nucleation and growth, Avrami wrote a series of papers to detail the theory and kinetic representation of nucleation and growth [60] [61] [62]. This behavior is described as the JMAEK approximation in Table 4.1. In summary, it is assumed that a number of random nucleation sites called germ nuclei, are induced in the matrix of a metal oxide. These can be considered as active sites for phase change and with the oxygen carrier reduction the germ nuclei are most likely oxygen vacancies. These germ nuclei are randomly distributed through the volume of the material and upon phase change, they are converted to growth nuclei and continue to grow until the growth nuclei eventually coalesce thus converting the material into the product phase. Throughout the duration of the reaction, the growth nuclei are competing to convert the remaining active phase and Avrami postulated that the ratio of a grain without competing growth 
nuclei impingement to a grain impinged by overlapping growth nuclei was equal to the fraction of the remaining unreacted phase represented by Equation 4.17 where $X$, and $X_{\text {ex }}$ represent the fractional conversions of grains with and without overlapping, or impingement, respectively. In the nomenclature defined by Avrami, theoretical grains without overlapping growth nuclei were called the "extended volume."

$$
\frac{d X_{e x}}{d X}=\frac{1}{1-X}
$$

Upon integration of Equation 4.17 a function that relates the rate determining mechanism of growth can be represented to account for the variations in reactivity due to the rapidly diminishing unreacted material caused by the increasing influence of growth nuclei. As the ability of growth nuclei to convert the unreacted regions decreases, the rate of phase change correspondingly decreases thus producing the characteristic sigmoidal curve of nucleation and growth kinetics shown by the integration of Equation 4.17 to Equation 4.18 and the algebraic rearrangement to Equation 4.19.

$$
\begin{aligned}
& X_{e x}=-\ln (1-X) \\
& X=1-e^{-X_{e x}}
\end{aligned}
$$

It has been speculated that any function from the column labeled $f(t)$ in Error! Reference source not found. could be inserted as a function equal to $X_{e x}$, however, the power law is the most common function introduced and this yields the JMAEK equation previously shown in Error! Reference source not found. 


\subsection{Model-Fitting and Model-Free Analysis}

For model-fitting analysis, the rate equations obtained from derivations found in literature are compared with experimental weight change data obtained from TGA experiments and the closeness of fit is evaluated. In this analysis, the established derivations listed in column $f(X)$ in Error! Reference source not found., and briefly described earlier, are differentiated with respect to time in Equation 4.20 to yield the equations in column $g(X)$ which are represented by Equation 23. This equation is algebraically rearranged to yield the expression in column $g^{-1}(X)$ in Error! Reference source not found. where closeness of fit can be evaluated between the model and any given experimental conversion data using MATLAB curve-fitting software.

$$
\begin{gathered}
\int(k) d \tau=\int \frac{1}{f(X)} d X \\
\int \frac{1}{f(X)} d X=g(X)
\end{gathered}
$$

Determination of important kinetic parameters such as $\mathrm{k}_{0}$, frequency factor, and $\mathrm{E}_{\mathrm{a}}$, activation energy, were also investigated using the Arrhenius Equation, as shown in Equation 4.22, for the reaction rate " $\mathrm{k}$ " which is derived during model-analysis in MATLAB.

$$
k(T)=k_{0} e^{\frac{-E_{a}}{R T}}
$$

Equation 4.22 was substituted into $g^{-1}(\tau)$ for " $\mathrm{k}$ " to observe the temperature dependence of each reaction. Activation energy and frequency factor were determined by fitting the plots of $\ln (k) v s \cdot \frac{1}{T}$ for the desired temperature range.

For model-free analysis, an assumption of the reaction mechanism is not required thus avoiding any analytical error in the determination of activation energy. By assuming the reaction 
takes the form of Equation 4.23, and rearrangement to Equation 4.24, activation energy can be calculated for a given set of temperature and conversion.

$$
\begin{gathered}
g(X)=k_{0} e^{\frac{-E a}{R T}} t \\
\ln (t)=\ln g(X)-\ln \left(k_{0}\right)+\frac{E_{a}}{R T}
\end{gathered}
$$

Where $g(X), k_{0}, E_{a}, T$, and $t$ represent conversion, frequency factor, activation energy, temperature and time. After linear regression of fitted $\mathrm{t}$, and $\mathrm{T}$ data in Equation 4.24, the activation energy for a given conversion can be determine from the slope of this function. 


\section{Chapter 5. Design of a High Performing Oxygen Carrier}

\subsection{Formulation}

Formulation is an important aspect of oxygen carrier development. As mentioned, although the pure metal oxides exhibited promising features of a high performing oxygen carrier, a diverse range of problems limited the practical application of these carriers. However, the desirable features of these components can be incorporated into multicomponent carriers to develop a highperforming carrier. The discovery and optimization of the trimetallic formulation was critical in increasing the overall global reaction rate.

As shown by the global reaction rate maxima data in Figure 5.1 and Figure 5.2 the trimetallic phase and the composition of the phase were important parameters that influenced reactivity.
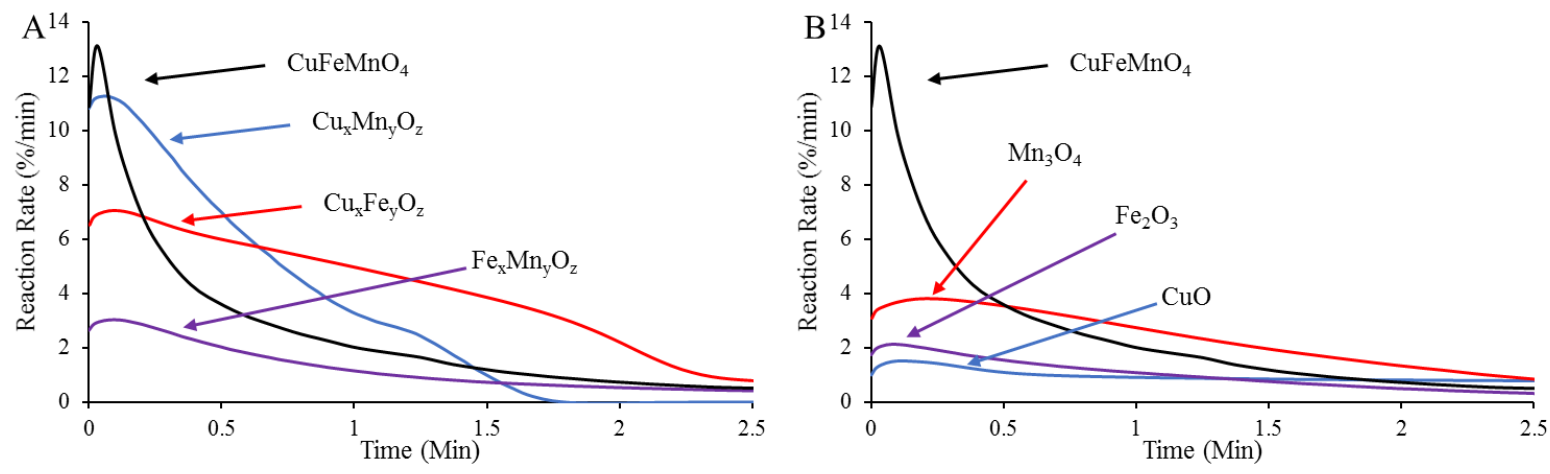

Figure 5.1 Global rates of reaction comparison of $\mathrm{CuFeMnO}_{4}$ and its (A) bimetallic and (B) single metallic counterparts obtained from 10th TGA reduction cycle at $800{ }^{\circ} \mathrm{C}$

According to the data in Figure 5.1A and Figure 5.1B, $\mathrm{CuFeMnO}_{4}$ achieved the highest global reaction rate during the 10 th reduction reaction with $\mathrm{CH}_{4}$ during multi-cycle TGA experiments at $800{ }^{\circ} \mathrm{C}$ compared to its single and bimetallic counterparts. The global reaction maxima were calculated by differentiating the weight loss data with respect to time while the oxygen carrier is exposed to $20 \% \mathrm{CH}_{4}$ balance Ar. According to the data shown in Figure 5.1A, 
the trimetallic, bimetallic copper iron oxide, and bimetallic copper manganese oxide demonstrated improved global reaction rates compared to that of the single metal oxides. However, increased reactivity was not exclusive to complex metal oxide systems as iron manganese oxide possessed very low reactivity like that of the single metal oxides. Thus, the formation of the trimetallic OC enhanced reactivity with $\mathrm{CH}_{4}$ and out-performed its counterpart metal oxide systems.

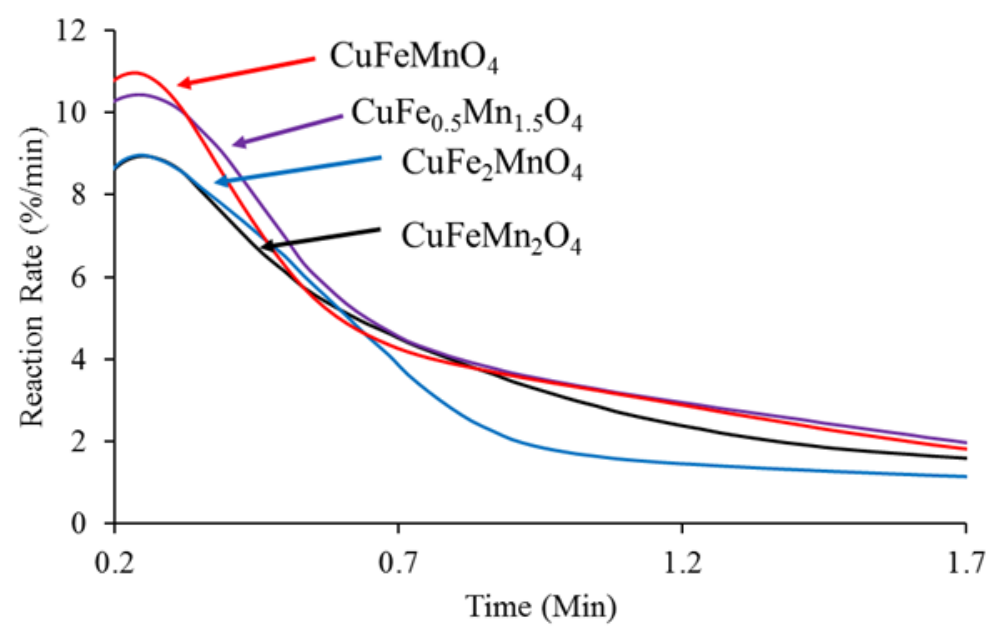

Figure 5.2. Global reaction rate comparison of various formulations of the complex trimetallic compositions during the 10th TGA reduction cycle

According to the data shown in Figure 5.2, bulk composition of the metal cation species influenced global reaction rate data as well. The global reaction rate maxima calculated during isothermal TGA $20 \% \mathrm{CH}_{4}$ balance Ar reductions at $800{ }^{\circ} \mathrm{C}$ with various compositions of the trimetallic OC indicated that reactivity of the trimetallic phases was heavily dependent on an equimolar ratio of the metal species. It is interesting to note that increasing the ratio of $(\mathrm{Fe}+\mathrm{Mn}): \mathrm{Cu}$ decreased global reaction rates. Thus, the influence of composition on global reaction rate calculations during reduction with $\mathrm{CH}_{4}$ is highly dependent on the stabilization of the $\mathrm{CuFeMnO}_{4}$ phase. 


\subsection{Reactivity}

The reactivity of $\mathrm{CuFeMnO}_{4}$ was further scrutinized with FB-MS and TGA-MS analysis using $\mathrm{CH}_{4}$ conversion and oxygen transfer capacity calculations.
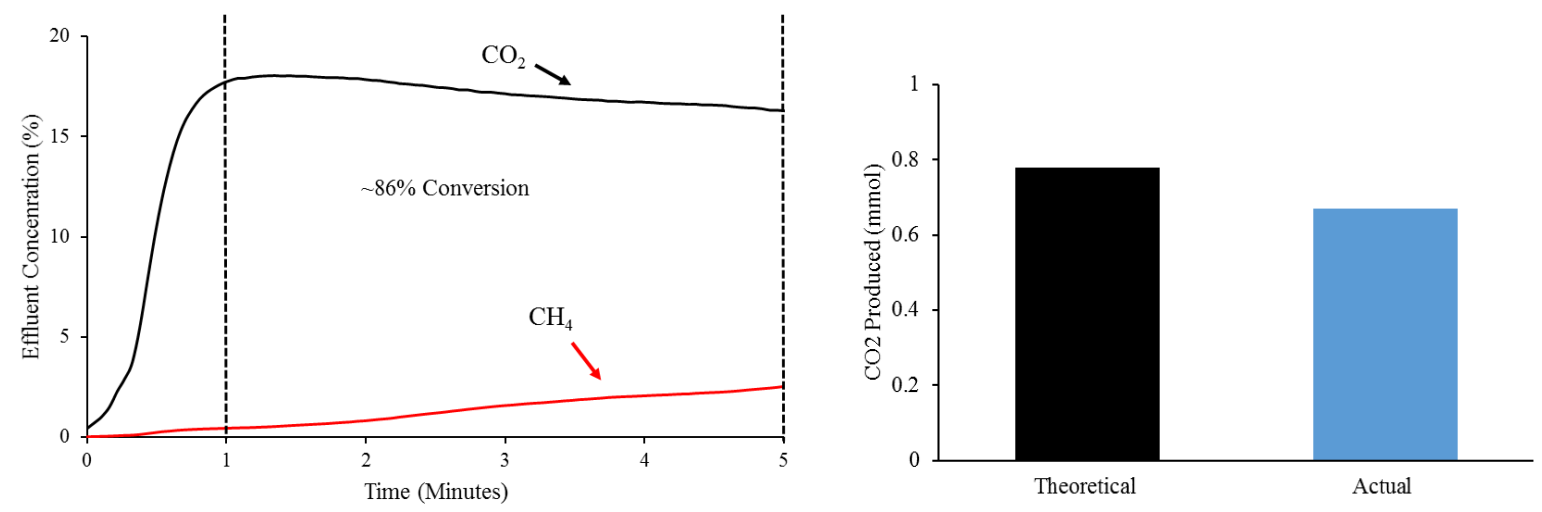

Figure 5.3. Isothermal fixed bed flow data during the 10th reduction of $\mathrm{CuFeMnO}_{4}$ with a comparison of the theoretical and experimental $\mathrm{mmol}$ of $\mathrm{CO}_{2}$ produced

According to the data in Figure 5.3, fixed bed isothermal MS data demonstrated the high $\mathrm{CH}_{4}$ consumption and conversion of $\mathrm{CuFeMnO}_{4}$ during reduction with $\mathrm{CH}_{4}$. $\mathrm{CuFeMnO} 4$ achieved $86 \% \mathrm{CH}_{4}$ conversion to $\mathrm{CO}_{2}$ during the four-minute interval shown in Figure 5.3. This interval was chosen to allow the reaction inside the reactor enough time to reach equilibrium and account for the possibility of varying $\mathrm{CH}_{4}$ concentration during the initiation of the reduction cycle. Fixed bed isothermal experiments indicated that $\mathrm{CuFeMnO}_{4}$ converted a large majority of $\mathrm{CH}_{4}$ to $\mathrm{CO}_{2}$ which is a very important achievement in the fuel reactor. Sequestration-ready $\mathrm{CO}_{2}$ produced in the fuel reactor must contain minimal flammable gases from incomplete oxidation of the reaction gases due to low reaction conversions. 


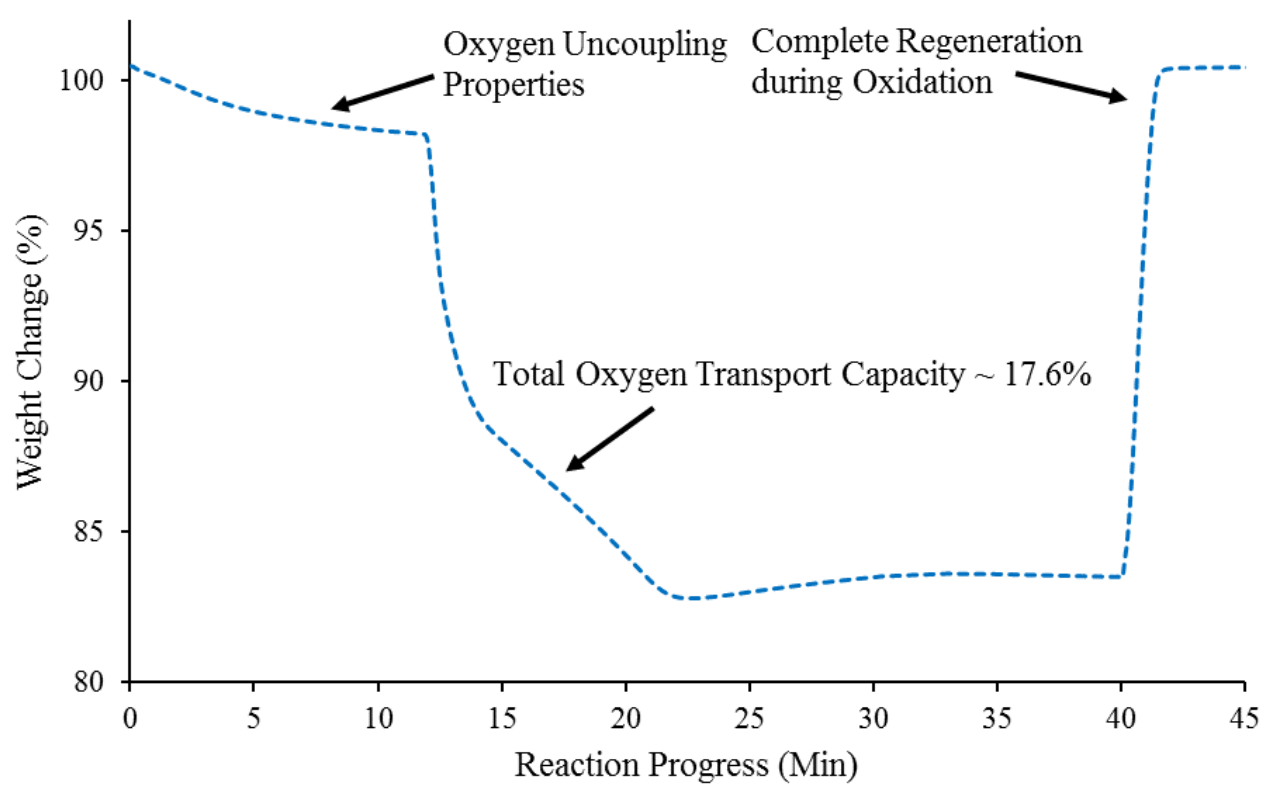

Figure 5.4. Reaction data of $\mathrm{CuFeMnO}_{4}$ during 10th TGA reduction cycle with $\mathrm{CH}_{4}$ In addition to increased reactivity, the trimetallic OC also possessed improved oxygen transfer capacity compared to other metal oxide systems according to the data in Figure 5.4. As mentioned previously, the reduction of iron oxide to metallic $\mathrm{Fe}$ in the presence of $\mathrm{CH}_{4}$ is not favorable [22]. However, the reduction of the trimetallic system produced metallic Fe during deeper levels of reduction with $\mathrm{CH}_{4}$ due to synergistic effects in the complex metal oxide system. In order to satisfy the mass balance in Table 5.1, the reduction of iron oxide and partially manganese oxide must occur to the respective metallic species, which was not observable in the single metal oxide systems. In addition to enhanced oxygen transfer capacity, the oxidation of $\mathrm{CuFeMnO}_{4}$ regenerated the complex system and deactivation was not observed. 
Table 5.1 Estimated oxygen transfer capacities for the copper manganese iron oxide system

\begin{tabular}{|c|c|c|}
\hline Reaction & $\begin{array}{c}\text { Theoretical } \\
\text { Oxygen Transfer }\end{array}$ & $\begin{array}{c}\text { Experimental } \\
\text { Oxygen Transfer }\end{array}$ \\
\hline $\mathrm{CuO}+\mathrm{Fe}_{3} \mathrm{O}_{4}+\mathrm{Mn}_{3} \mathrm{O}_{4}+2 \mathrm{CH}_{4} \rightarrow \mathrm{Cu}+3 \mathrm{MnO}+3 \mathrm{Fe}+2 \mathrm{CO}_{2}+4 \mathrm{H}_{2} \mathrm{O}$ & $17.9 \%$ & $18.2 \%$ \\
\hline $\mathrm{CuFeMnO}_{4}+\mathrm{CH}_{4} \rightarrow \mathrm{CuFeMnO}_{2} / \mathrm{CuFeMnO}+\mathrm{CO}_{2}+2 \mathrm{H}_{2} \mathrm{O}$ & $13.4 \%-20.1 \%$ & $18.2 \%$ \\
\hline
\end{tabular}

Clearly, $\mathrm{CuFeMnO} 4$ possessed excellent reactivity and oxygen transport capacity during reactions with $\mathrm{CH}_{4}$. Preparation method/cost, reaction stability, and resistance to attrition are also important parameters in OC development and must be addressed if copper iron manganese is truly the ideal candidate for CLC. These aspects will be discussed in the following sections

\subsection{OC Preparation Economic Assessment}

Large-scale OC synthesis is an important part in CLC commercialization due to the size and throughput of OC's in a large-scale plant. As previously reported, $\mathrm{CuFeMnO}_{4}$, synthesized from the sol-gel method of metal nitrate salts, formed a promising trimetallic OC with excellent reactivity and stability demonstrated during isothermal experiments [21]. Typically, for complex metal oxide preparation, sol-gel, co-precipitation, or wet impregnation methods are common practice [70] [71]. These methods involve expensive raw materials and extensive wet chemistry procedures to form the complex mixed metal oxides and can form harmful by-products due to decomposition of the nitrate salt precursors during calcination. Therefore, this preparation method is not ideal for large-scale applications due to cost and environmental concerns. Thus, the tumbling method of metal oxide precursors was adopted which replicated a commercial preparation process that produces taconite pellets from raw iron ores [72]. This method uses only water to act as a binder while the metal oxide precursors form spherical pellets in a rotary mixer in the desired size range. The pellets are then calcined to form the complex metal oxide phases and improve resistance to attrition. 


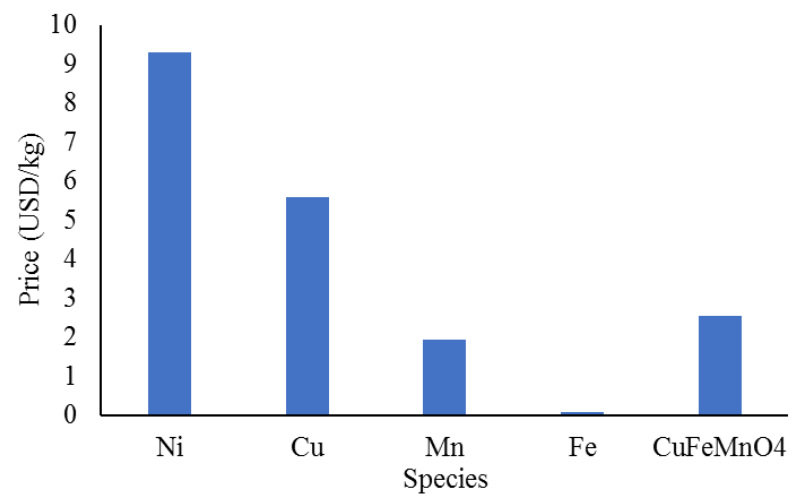

Figure 5.5. Cost analysis of metal species and the theoretical cost of manufacturing $\mathrm{CuFeMnO}_{4}$

In the present study, $\mathrm{CuFeMnO}_{4}$ is comprised of inexpensive and benign metals in an environmentally conscious preparation procedure that does not sacrifice performance unlike that of $\mathrm{NiO}$ which was among the initial OC candidates selected for CLC [73]. Although NiO may possess high reactivity with $\mathrm{CH}_{4}$, the market value of $\mathrm{Ni}$ is $9.31 \mathrm{USD} / \mathrm{kg}$ compared to that of 5.59 $\mathrm{USD} / \mathrm{kg}$ for $\mathrm{Cu}, 1.92 \mathrm{USD} / \mathrm{kg}$ for $\mathrm{Mn}$, and $0.07 \mathrm{USD} / \mathrm{kg}$ for iron ore pellets according to the data in in Figure 5.5 [74] [75]. In addition to the high-cost associated with $\mathrm{Ni}$, this element is a suspected carcinogen and the risks of health issues due to exposure will increase manufacturing and operating costs as well [76].

A large majority of raw iron ore is pelletized and indurated before shipment in a commercial process commonly used in taconite processing facilities and the costs associated with mining, and production can be estimated at $0.04 \mathrm{USD} / \mathrm{kg}$ [72]. Based off the known values of this process which reaches calcination temperatures and particle diameters similar to that of an OC, the cost to produce the trimetallic OC was estimated assuming that the trimetallic OC would be produced in the same manner as the taconite pellets and the cost of the precursor components was determined from market value analysis. According to the cost assessment in Figure 5.5, the cost to fabricate the trimetallic OC was estimated to be $2.53 \mathrm{USD} / \mathrm{kg}$ given today's market value which 
is well below the cost of nickel oxide. This value falls in an economically viable range for CLC operation as compared to that of other synthetic $\mathrm{Cu}$ - and $\mathrm{Fe}-$ based $\mathrm{OC}$ [77].

\subsection{Attrition/Chemical Stability}

Resistance to attrition and reaction stability were investigated using an ASTM standard experiment in a jet-cup attrition unit and multi-cycle FB-MS experiments respectively.

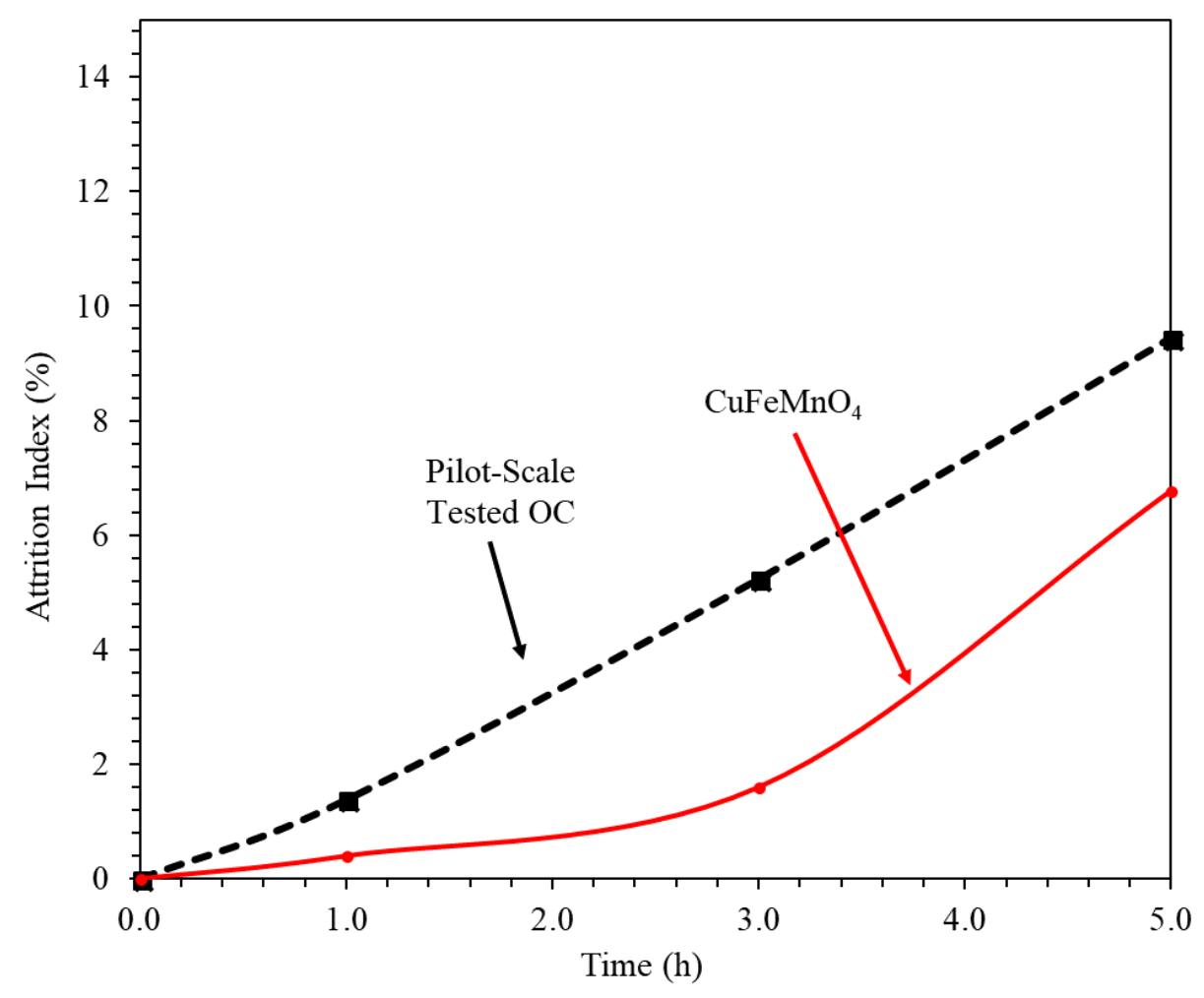

Figure 5.6. Comparison of attrition index data between an $\mathrm{OC}$ which has been tested in a pilotscale reactor and $\mathrm{CuFeMnO}_{4}$

According to the data provided in Figure 5.6, $\mathrm{CuFeMnO}_{4}$ demonstrated excellent resistance to physical attrition compared to that of an OC which has been tested in a pilot-scale reactor system during attrition testing. During this experiment, the oxygen carrier was exposed to velocities greater than $300 \mathrm{~m} / \mathrm{s}$ in a jet-cup fluidized bed at room temperature. These flows are well above any which an oxygen carrier may experience in a chemical looping reactor system. 
These extreme conditions force the oxygen carrier particles to collide with high momentum so that mass losses from abrasion and cracking can be studied. As shown by the data in Figure 5.6, minimal mass loss can be observed during attrition index testing of the trimetallic OC. The oxygen carrier demonstrated exemplary resistance to attrition in this jet-cup fluidized bed set up. The data provided from this experiment concluded that the forces of abrasion and cracking, which typically hinder the development of oxygen carriers, were minimally observed. The enhanced resistance to attrition demonstrated by the trimetallic oxygen carrier suggested that this carrier has the potential to withstand the physical degradation that could be experienced during CLC operation with improved success compared to that of an OC which has been tested in a pilot-scale reactor system.

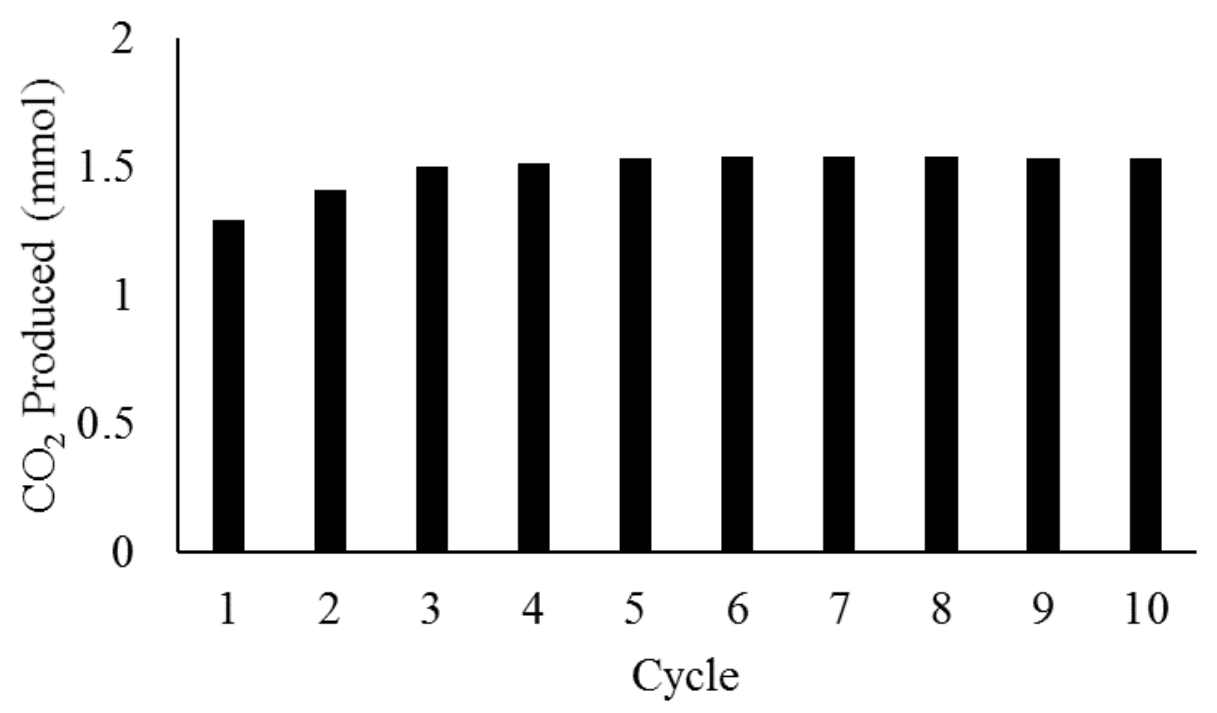

Figure 5.7. Reaction stability of $\mathrm{CuFeMnO}_{4}$ during 10 isothermal fixed bed $\mathrm{CH}_{4}$ reduction/ air oxidation cycles at $800^{\circ} \mathrm{C}$

According to the data in

Figure 5.7, $\mathrm{CuFeMnO}_{4}$ demonstrated excellent reaction stability. Approximately $1 \mathrm{~g}$ of the trimetallic OC was loaded into a fixed bed reactor with alternating $20 \% \mathrm{CH}_{4}$ balance $\mathrm{He}$ and air 
exposures at $800{ }^{\circ} \mathrm{C}$. $\mathrm{CH}_{4}$ conversion to $\mathrm{CO}_{2}$ was consistent as demonstrated by the repeatable molar production of $\mathrm{CO}_{2}$ during the cyclic test. According to the reaction data in

Figure 5.7, agglomeration due to the close proximity of the particles loaded in the fixed bed setting or reaction deactivation was not demonstrated by $\mathrm{CuFeMnO}_{4}$ due to the consistent production of $\mathrm{CO}_{2}$ produced during the reduction reaction. As evidenced by the data provided in Figure 5.6 and Figure 5.7 $\mathrm{CuFeMnO}_{4}$ demonstrated excellent reaction stability and resistance to attrition and thus, copper iron manganese oxide is an ideal candidate for CLC. 


\section{Chapter 6. Characterization}

\subsection{Assessment of Enhanced Reactivity}

The previous reactivity screening demonstrated that trimetallic $\mathrm{CuFeMnO}_{4}$ exhibits enhanced performance during CLC conditions. However, the details of this enhanced reactivity were not well understood. XRD and BET analysis were conducted to observe any reactive features that could be contributing the high reactivity of the promising OC.

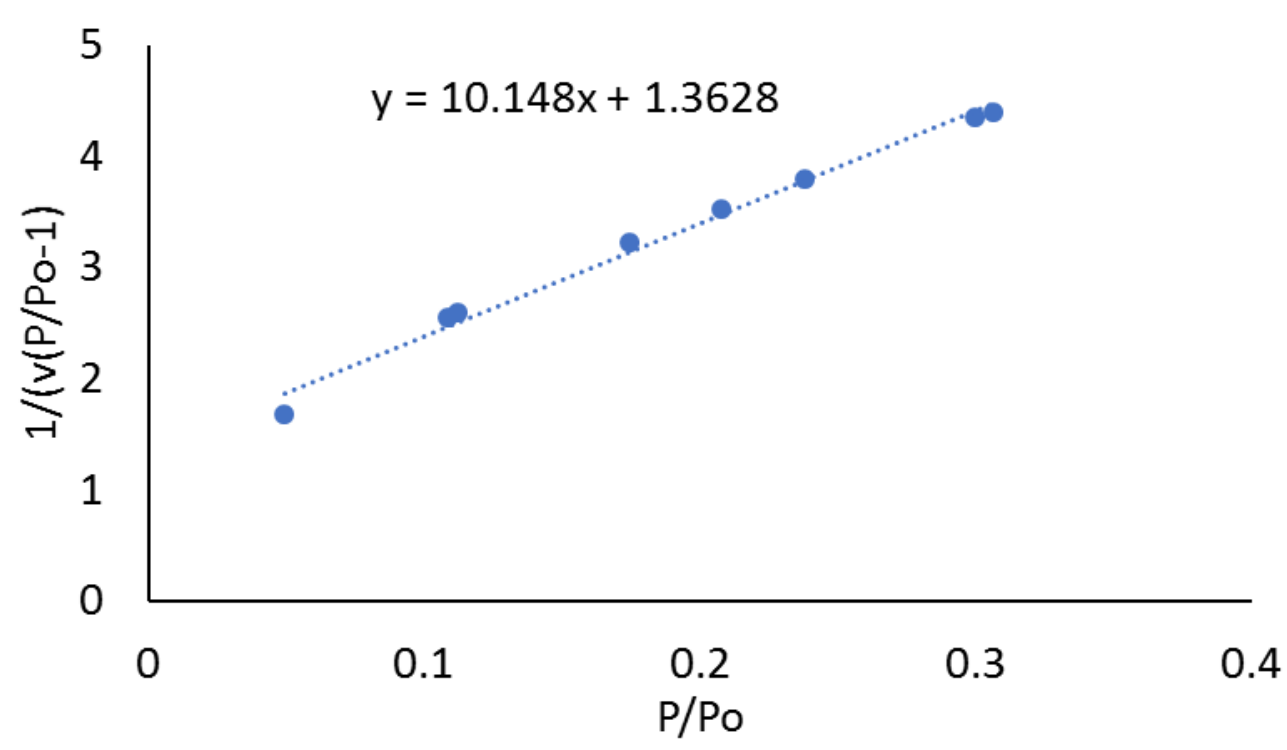

Figure 6.1 BET surface area plot of $\mathrm{CuFeMnO}_{4}$

As shown by the data in Figure 6.1, the trimetallic carrier demonstrated a BET surface area of $0.38 \mathrm{~m}^{2} / \mathrm{g}$. Although this surface area is particularly low compared to other values in traditional catalysis, it is common for oxygen carriers to possess surface areas less than $1 \mathrm{~m}^{2} / \mathrm{g}$ [24]. It was evident that surface area was a not major contribution to the enhanced reactivity and that the bulk composition of the complex metal oxide played a vital role in the performance enhancement as demonstrated by the earlier analysis. 
$\mathrm{XRD}$ analysis was then conducted on the trimetallic OC to observe the composition of the reactive bulk phase or phases that could be contributing to the excellent performance of the OC. $\mathrm{XRD}$ is the typical method used to identify the reactive mixed metal oxide phases.

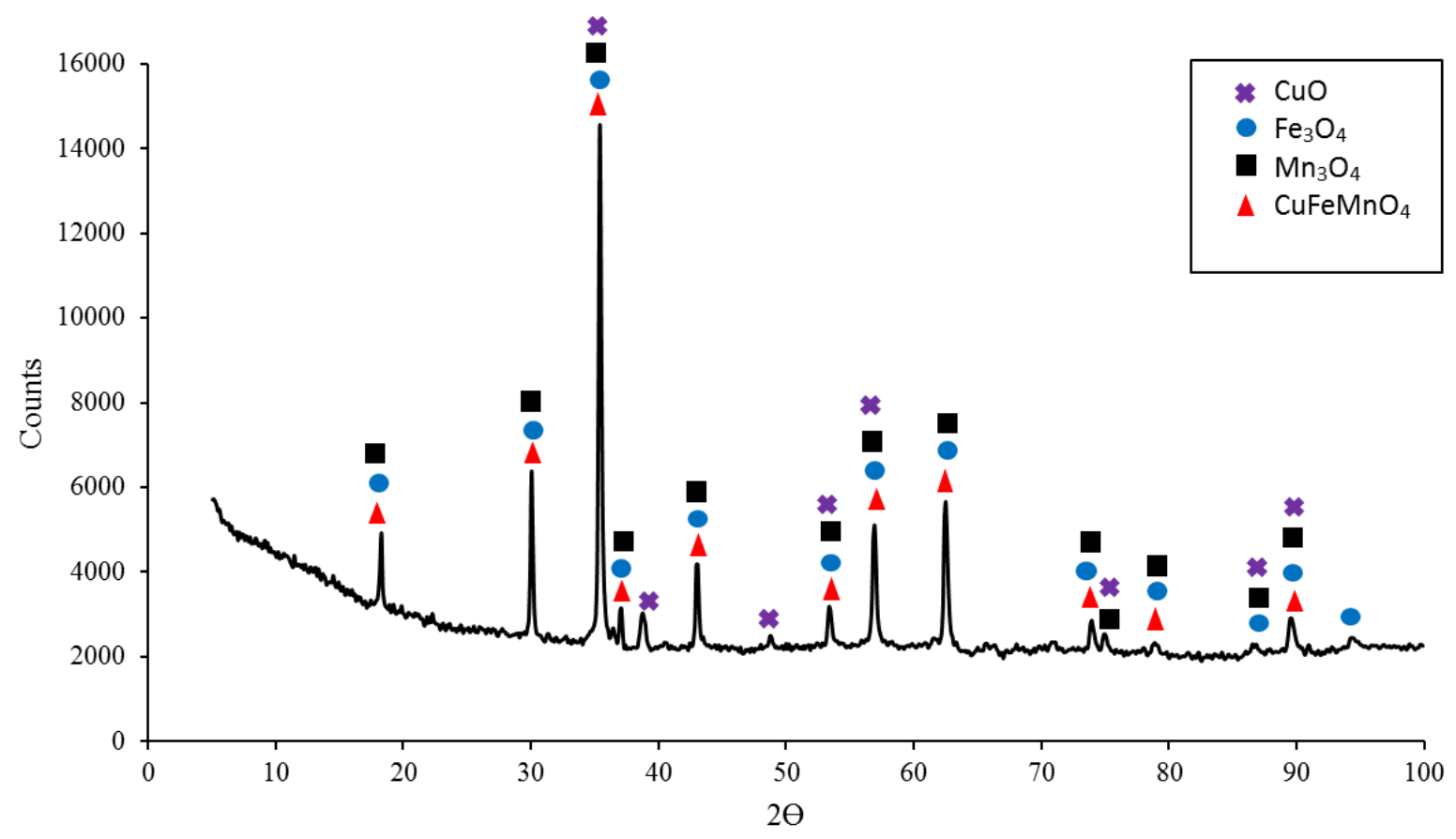

Figure 6.2. XRD pattern of freshly calcined trimetallic $\mathrm{CuFeMnO}_{4}$

However as shown in Figure 6.2, XRD analysis could not conclusively differentiate the characteristic peaks of $\mathrm{CuFeMnO}_{4}$, and its single metal counterparts; $\mathrm{CuO}, \mathrm{Fe}_{2} \mathrm{O}_{3}$, and $\mathrm{Mn}_{3} \mathrm{O}_{4}$. Therefore, the highly reactive phase formed was unable to be determined using standard characterization techniques and further questioned whether the enhanced observed reactivity was due to the formation of the trimetallic or due to the contribution of the pure metal oxides. It a multifaceted effort, the reduction pathway of $\mathrm{CuFeMnO}_{4}$ was studied to differentiate the unique trimetallic phase from its single and bimetallic counterparts and to gain a deeper understanding of the synergistic effects of the trimetallic system. The reaction rate and thermochemical data during controlled temperature ramps reported in this present study provided a means to distinguish metal oxide phases by comparing the unique reaction pathways. 
6.2. DSC/TGA during TPR with $\mathrm{CH}_{4}$ Performance Data Comparison of $\mathrm{CuFeMnO}_{4}$ and Corresponding Pure Metal Oxides

As shown by the heat flow, weight change, and reaction rate data in Figure 6.3A-C, DSC/TGA performance data during $16.6 \% \mathrm{CH}_{4}$ balance Ar TPR were conducted to compare the reduction pathway of $\mathrm{CuFeMnO} 4$ and the corresponding single metal counterparts.
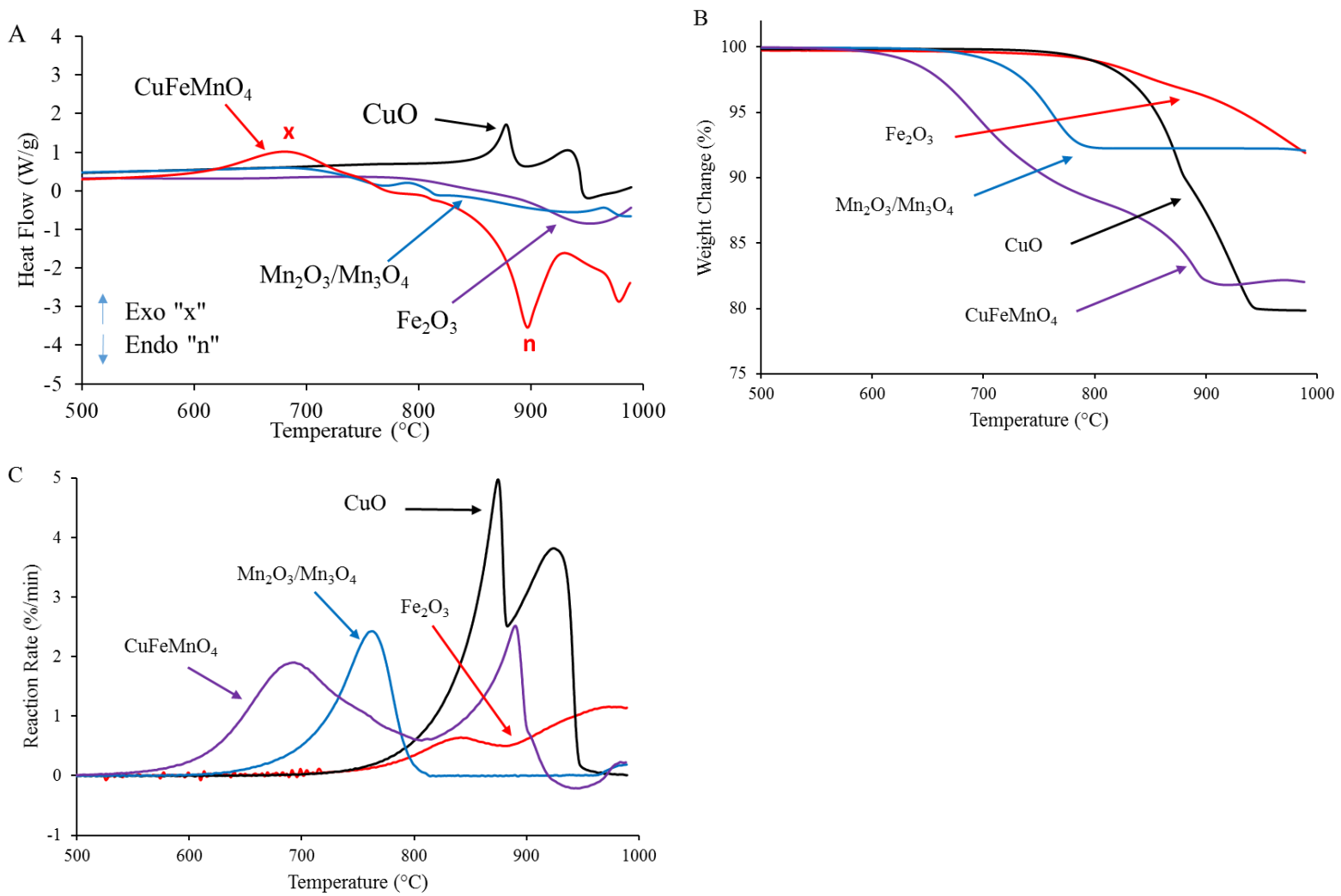

Figure 6.3. DSC/TGA during TPR with $16.6 \% \mathrm{CH}_{4}$ balance Ar performance data comparison of $\mathrm{CuFeMnO}_{4}$ and pure single metal counterparts

According to the thermochemical and reaction performance data in Figure 6.3, $\mathrm{CuFeMnO}_{4}$ exhibited a unique reduction pathway that was not observed in any of the pure single metal counterparts. In Figure 6.3A, the characteristic heat flow produced during the reaction between the $\mathrm{OC}$ and $\mathrm{CH}_{4}$ can be observed where " $\mathrm{x}$ " represents exothermic heat flow and " $n$ " represents endothermic heat flow. It should be noted that the thermochemical data agreed qualitatively with FactSage calculations shown in Table 6.1. This characteristic heat flow produced from these 
reactions correlated with reaction rate maxima, shown in Figure 6.3C. Reaction initiation temperatures, thermochemical data, and reaction rate data distinguished a unique reduction pathway inherent to the trimetallic system that did not possess any single metal oxide phases. In addition to the simultaneous DSC/TGA data, temperature programmed reduction further distinguished differences in reduction pathways that may not be observable in isothermal experiments. Thus, the experimental setup offered an advantageous way of differentiating the trimetallic OC from its single metal oxides due to the characteristic reduction pathway in $\mathrm{CH}_{4}$.

Table 6.1. Theoretical enthalpy of reaction calculation with pure single metal oxides and $\mathrm{CH}_{4}$

\begin{tabular}{|c|c|}
\hline Reaction & $\Delta \mathrm{H}_{800}(\mathrm{KJ})$ \\
\hline $8 \mathrm{CuO}_{4} \mathrm{CH}_{4} \rightarrow 4 \mathrm{Cu}_{2} \mathrm{O}+\mathrm{CO}_{2}+2 \mathrm{H}_{2} \mathrm{O}$ & $-33.9 \mathrm{KJ}$ \\
\hline $4 \mathrm{Cu}_{2} \mathrm{O}+\mathrm{CH}_{4} \rightarrow 8 \mathrm{Cu}+\mathrm{CO}_{2}+2 \mathrm{H}_{2} \mathrm{O}$ & $-33.0 \mathrm{KJ}$ \\
\hline $12 \mathrm{Fe}_{2} \mathrm{O}_{3}+\mathrm{CH}_{4} \rightarrow 8 \mathrm{Fe}_{3} \mathrm{O}_{4}+\mathrm{CO}_{2}+2 \mathrm{H}_{2} \mathrm{O}$ & $+14.3 \mathrm{KJ}$ \\
\hline $4 \mathrm{Fe}_{3} \mathrm{O}_{4}+\mathrm{CH}_{4} \rightarrow 12 \mathrm{FeO}+\mathrm{CO}_{2}+2 \mathrm{H}_{2} \mathrm{O}$ & $+98.6 \mathrm{KJ}$ \\
\hline $4 \mathrm{FeO}+\mathrm{CH}_{4} \rightarrow 4 \mathrm{Fe}+\mathrm{CO}_{2}+2 \mathrm{H}_{2} \mathrm{O}$ & $+64.2 \mathrm{KJ}$ \\
\hline $12 \mathrm{Mn}_{2} \mathrm{O}_{3}+\mathrm{CH}_{4} \rightarrow 8 \mathrm{Mn}_{3} \mathrm{O}_{4}+\mathrm{CO}_{2}+2 \mathrm{H}_{2} \mathrm{O}$ & $-42.7 \mathrm{KJ}$ \\
\hline $4 \mathrm{Mn}_{3} \mathrm{O}_{4}+\mathrm{CH}_{4} \rightarrow 12 \mathrm{MnO}+\mathrm{CO}_{2}+2 \mathrm{H}_{2} \mathrm{O}$ & $+28.4 \mathrm{KJ}$ \\
\hline
\end{tabular}

As mentioned, the thermochemical data in Figure 6.3A qualitatively agreed with the calculated enthalpies of reaction between single metal oxides and $\mathrm{CH}_{4}$ in Table 6.1. The thermochemical data obtained from these experiments confirmed that $\mathrm{CuO}$ has an exothermic reaction with $\mathrm{CH}_{4}$, but that the exothermic reaction of $\mathrm{CuO}$ was not observable in the trimetallic system. The exothermic reaction between $\mathrm{CuFeMnO}_{4}$ and $\mathrm{CH}_{4}$ indicated that the trimetallic system produced a new exothermic reaction that was not due to bulk or surface $\mathrm{CuO}$ distributed in the trimetallic system. Furthermore, an exothermic reduction validated that a CLC reactor operating with this trimetallic OC could sustain auto-thermal operation with greater ease. If the reducing reactor can maintain temperature during continuous CLC, then most of the heat from the oxidizing 
reactor can go straight to energy production without requiring any heat transfer back to the reducing reactor, which tremendously simplifies reactor design.

Table 6.2. Experimental and theoretical oxygen transfer capacities of the single metal oxides and $\mathrm{CuFeMnO}_{4}$

\begin{tabular}{|c|c|c|}
\hline Reaction & $\begin{array}{c}\text { Theoretical } \\
\text { Oxygen Transfer }\end{array}$ & $\begin{array}{c}\text { Experimental } \\
\text { Oxygen Transfer }\end{array}$ \\
\hline $\mathrm{CuO}+\mathrm{Fe}_{3} \mathrm{O}_{4}+\mathrm{Mn}_{3} \mathrm{O}_{4}+2 \mathrm{CH}_{4} \rightarrow \mathrm{Cu}+3 \mathrm{MnO}+3 \mathrm{Fe}+2 \mathrm{CO}_{2}+4 \mathrm{H}_{2} \mathrm{O}$ & $17.9 \%$ & $18.2 \%$ \\
\hline $\mathrm{CuFeMnO} \mathrm{CH}_{4}+\mathrm{CH}_{4} \rightarrow \mathrm{CuFeMnO}_{2} / \mathrm{CuFeMnO}+\mathrm{CO}_{2}+2 \mathrm{H}_{2} \mathrm{O}$ & $13.4 \%-20.1 \%$ & $18.2 \%$ \\
\hline $6 \mathrm{CuO}+\mathrm{CH}_{4} \rightarrow 3 \mathrm{Cu}_{2} \mathrm{O}+\mathrm{CO}_{2}+\mathrm{H}_{2} \mathrm{O}$ & $10.1 \%$ & $10.4 \%$ \\
\hline $4 \mathrm{CuO}+\mathrm{CH}_{4} \rightarrow 4 \mathrm{Cu}+\mathrm{CO}_{2}+2 \mathrm{H}_{2} \mathrm{O}$ & $20.1 \%$ & $20.1 \%$ \\
\hline $9 \mathrm{Fe}_{2} \mathrm{O}_{3}+\mathrm{CH}_{4} \rightarrow 6 \mathrm{Fe}_{3} \mathrm{O}_{4}+\mathrm{CO}_{2}+2 \mathrm{H}_{2} \mathrm{O}$ & $3.3 \%$ & $3.3 \%$ \\
\hline $4 \mathrm{Fe}_{2} \mathrm{O}_{3}+\mathrm{CH}_{4} \rightarrow 8 \mathrm{FeO}+\mathrm{CO}_{2}+2 \mathrm{H}_{2} \mathrm{O}$ & $10.0 \%$ & $8.1 \%$ \\
\hline $4 \mathrm{Mn}_{2} \mathrm{O}_{3}+\mathrm{CH}_{4} \rightarrow 8 \mathrm{MnO}+\mathrm{CO}_{2}+2 \mathrm{H}_{2} \mathrm{O}$ & $10.1 \%$ & $7.9 \%$ \\
\hline
\end{tabular}

Experimental oxygen transfer capacity was calculated from the experimental reaction data in Figure 6.3B. The experimental and theoretical oxygen transfer capacities were compared to estimate extent of reduction during the reaction with $\mathrm{CH}_{4}$. As shown in Table 6.2, $\mathrm{CuO}$ was fully reduced to its metallic species in both the trimetallic $\mathrm{CuFeMnO}_{4}$ and $\mathrm{CuO}$ experiments. However, during the TPR experiment with $\mathrm{Fe}_{2} \mathrm{O}_{3}$, metallic Fe was not produced. In addition, $\mathrm{Mn}_{2} \mathrm{O}_{3} / \mathrm{Mn}_{3} \mathrm{O}_{4}$ did not fully reduce to its metallic species as well. As shown by the results in Table 6.2, a synergistic interaction among the metal oxides in the trimetallic system formed metallic Fe and a small amount of metallic Mn which was not evident in the single metal analysis. The performance data in Figure 6.3, and the reaction data in Table 6.2 clearly distinguished $\mathrm{CuFeMnO}_{4}$ from its single metal counterparts. $\mathrm{CuFeMnO}_{4}$ was further scrutinized with its bimetallic counterparts to confirm that a unique trimetallic phase was synthesized after calcination. 
6.3. DSC/TGA during TPR with $\mathrm{CH}_{4}$ Performance Data Comparison of $\mathrm{CuFeMnO}_{4}$ and Corresponding Pure Metal Oxides

As shown by the heat flow, weight change, and reaction rate data in Figure 6.4A-C, DSC/TGA performance data during $16.6 \% \mathrm{CH}_{4}$ balance $\mathrm{Ar}$ TPR were conducted to compare the reduction pathway of $\mathrm{CuFeMnO} 4$ and the corresponding bimetallic counterparts.
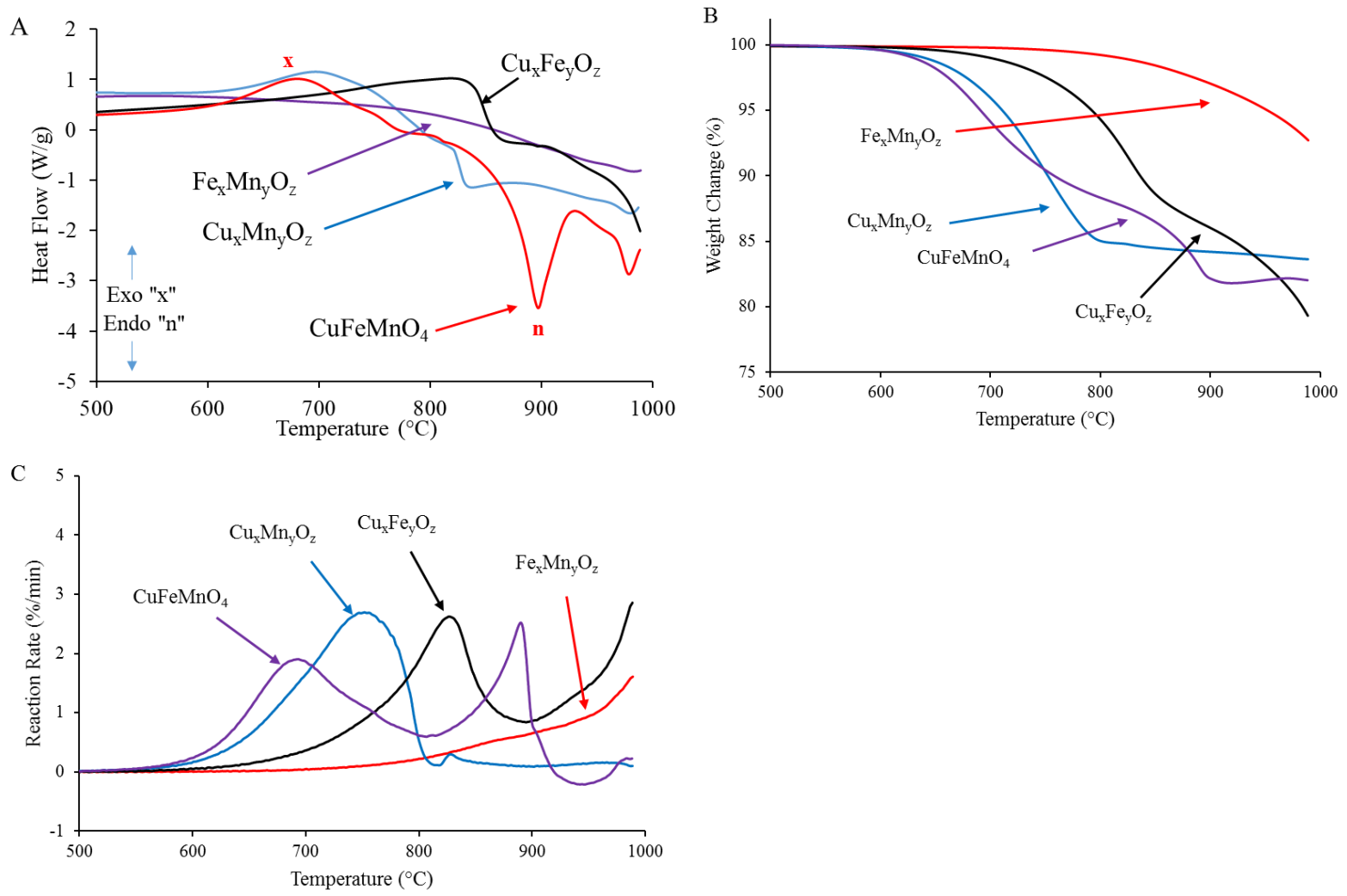

Figure 6.4. DSC/TGA during TPR with $16.6 \% \mathrm{CH}_{4}$ balance Ar performance data comparison of $\mathrm{CuFeMnO}_{4}$ and bimetallic counterparts

A comparison of the performance data obtained from simultaneous DSC/TGA TPR with $\mathrm{CH}_{4}$ experiments of $\mathrm{CuFeMnO}_{4}$ and its bimetallic counterparts is shown in Figure 6.4. According to the data shown in Figure $6.4 \mathrm{~A}, \mathrm{CuFeMnO}$ produced characteristic heat flow during TPR with $\mathrm{CH}_{4}$. The reduction pathway obtained from these characteristic heat flow and reaction rate data did not indicate that the bimetallic counterparts represented a dominant phase in the trimetallic system. Thus, it was concluded that $\mathrm{CuFeMnO}_{4}$ forms a unique phase after calcination and the 
enhanced reactivity with $\mathrm{CH}_{4}$ can be attributed to the formation of a unique trimetallic oxide system and not to the interaction of single or bimetallic counterparts.

Table 6.3. Experimental and theoretical oxygen transfer capacities of the bimetallic oxides and $\mathrm{CuFeMnO}_{4}$

\begin{tabular}{|c|c|c|}
\hline Reaction & $\begin{array}{c}\text { Theoretical } \\
\text { Oxygen Transfer }\end{array}$ & $\begin{array}{c}\text { Experimental } \\
\text { Oxygen Transfer }\end{array}$ \\
\hline $\mathrm{CuO}+\mathrm{Fe}_{3} \mathrm{O}_{4}+\mathrm{Mn}_{3} \mathrm{O}_{4}+2 \mathrm{CH}_{4} \rightarrow \mathrm{Cu}+3 \mathrm{MnO}+3 \mathrm{Fe}+2 \mathrm{CO}_{2}+4 \mathrm{H}_{2} \mathrm{O}$ & $17.9 \%$ & $18.2 \%$ \\
\hline $\mathrm{CuFeMnO}+\mathrm{CH}_{4} \rightarrow \mathrm{CuFeMnO}_{2} / \mathrm{CuFeMnO}+\mathrm{CO}_{2}+2 \mathrm{H}_{2} \mathrm{O}$ & $13.4 \%-20.1 \%$ & $18.2 \%$ \\
\hline $\mathrm{CuO}+\mathrm{Fe}_{2} \mathrm{O}_{3}+\mathrm{CH}_{4} \rightarrow \mathrm{Cu}+\mathrm{FeO} / \mathrm{Fe}+\mathrm{CO}_{2}+2 \mathrm{H}_{2} \mathrm{O}$ & $15.1 \%-25.1 \%$ & $20.6 \%$ \\
\hline $2 \mathrm{CuO}+2 \mathrm{Mn}_{2} \mathrm{O}_{3}+\mathrm{CH} 4 \rightarrow 2 \mathrm{Cu}+4 \mathrm{MnO}+\mathrm{CO}_{2}+2 \mathrm{H}_{2} \mathrm{O}$ & $15.1 \%$ & $16.4 \%$ \\
\hline $2 \mathrm{Fe}_{2} \mathrm{O}_{3}+2 \mathrm{Mn}_{2} \mathrm{O}_{3}+\mathrm{CH}_{4} \rightarrow 4 \mathrm{FeO}+4 \mathrm{MnO}+\mathrm{CO}_{2}+2 \mathrm{H}_{2} \mathrm{O}$ & $10.1 \%$ & $7.0 \%$ \\
\hline
\end{tabular}

Experimental oxygen transfer capacity was calculated from the experimental reaction data in Figure 6.4B. The experimental and theoretical oxygen transfer capacities were compared to estimate extant of reduction during the reaction with $\mathrm{CH}_{4}$. As shown in Table 6.3, the addition of $\mathrm{Cu}$ enhanced oxygen transfer capacity when incorporated with $\mathrm{Fe}_{2} \mathrm{O}_{3}$ and $\mathrm{Mn}_{2} \mathrm{O}_{3} / \mathrm{Mn}_{3} \mathrm{O}_{4}$ as demonstrated by the increased levels of reduction. The incorporation of $\mathrm{Cu}$ also promoted a new exothermic reaction with $\mathrm{CH}_{4}$. Bimetallic iron manganese oxide possessed the lowest oxygen transfer capacity, and highest reaction initiation temperature as shown in Table 6.3. The reduction of bimetallic iron manganese oxide also produced an exclusively endothermic reaction.

Table 6.4. Reaction temperatures of the exothermic and endothermic reaction of the various metal oxide systems

\begin{tabular}{|c|c|c|}
\hline Metal Oxide System & $\begin{array}{c}\text { Exotherm } \\
\text { Temperature }\left({ }^{\circ} \mathrm{C}\right)\end{array}$ & $\begin{array}{c}\text { Endotherm } \\
\text { Temperature }\left({ }^{\circ} \mathrm{C}\right)\end{array}$ \\
\hline Copper Oxide & 875,924 & None \\
\hline Iron Oxide & None & 842,973 \\
\hline Manganese Oxide & 763 & 821 \\
\hline Copper Iron Oxide & 827 & 81000 \\
\hline Copper Manganese Oxide & 751 & 827 \\
\hline Iron Manganese Oxide & None & 739 \\
\hline Separated Metal Oxides & 857,904 & 890 \\
\hline Copper Iron Manganese Oxide & 696 & \\
\hline
\end{tabular}


A summary of the thermochemical heat flow data and the associated temperature at the rate change maximum is shown in Table 6.4. Low temperature reduction and unique thermochemical data during DSC/TGA TPR with $\mathrm{CH}_{4}$ indicated that $\mathrm{CuFeMnO}_{4}$ formed a unique trimetallic phase and that did not contain single or bimetallic phases. The endothermic thermochemical data suggested the possibility of a solid-solid phase transition during reduction with $\mathrm{CH}_{4}$ and this was further investigated with in-situ XRD TPR with $\mathrm{CH}_{4}$.

\subsection{Solid-Solid Phase Transition}

As shown in Figure 6.5, the DSC heat flow data clearly indicated the stabilization of a unique phase that differed from any single or bimetallic counterpart. The characteristic reduction pathway of $\mathrm{CuFeMnO}_{4}$ demonstrated by the DSC data was further characterized using in-situ XRD during temperature programmed reductions.
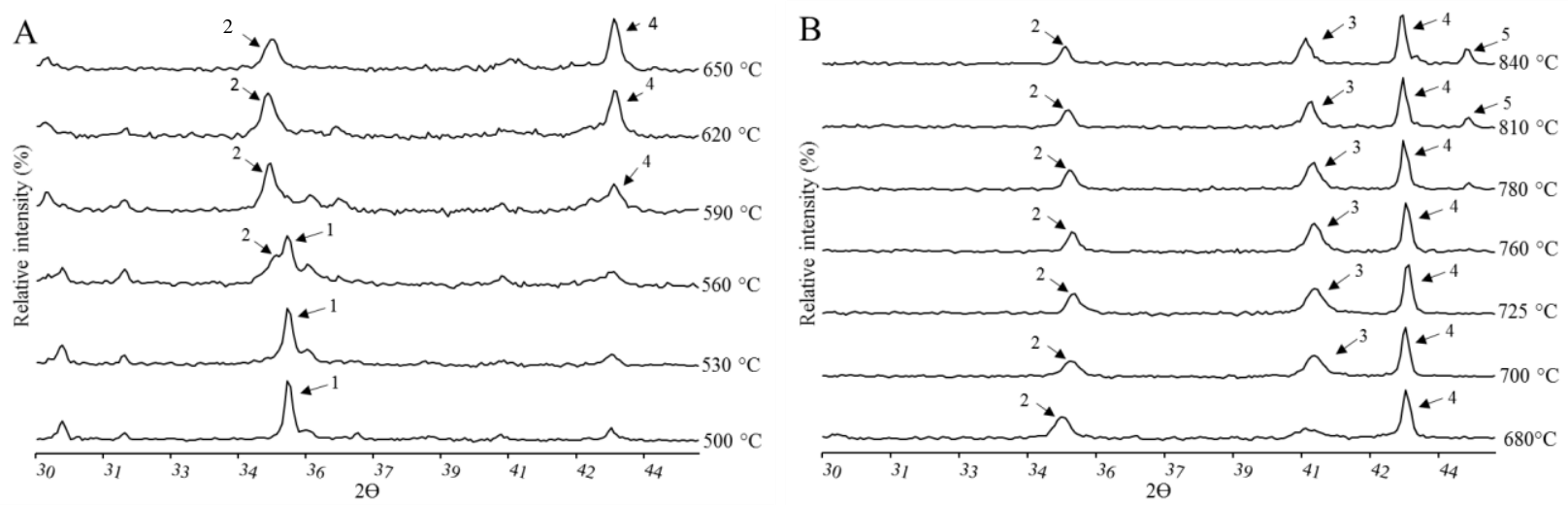

Figure 6.5. In-situ XRD analysis of $\mathrm{CuFeMnO}_{4}$ during temperature programmed reductions with $\mathrm{CH}_{4}$ : (A) $500{ }^{\circ} \mathrm{C}-650{ }^{\circ} \mathrm{C}$ (B) $670{ }^{\circ} \mathrm{C}-850{ }^{\circ} \mathrm{C}$; (1) $\mathrm{CuFeMnO}_{4}$, (2) $\mathrm{MnFe}_{2} \mathrm{O}_{4}$, (3) $\mathrm{MnO}$, (4) $\mathrm{Cu}$, (5) $\mathrm{Fe}$

The characteristic peak of $\mathrm{CuFeMnO}_{4}$, peak "1," is shown at lower temperatures in Figure 6.5A. This characteristic peak decreased as the temperature exceeded $550{ }^{\circ} \mathrm{C}$ and reduction in $\mathrm{CH}_{4}$ initiated. As the characteristic peak of $\mathrm{CuFeMnO}_{4}$ disappeared, peak “2," $\mathrm{MnFe}_{2} \mathrm{O}_{4}$, began to form at approximately $600{ }^{\circ} \mathrm{C}$. $\mathrm{MnFe}_{2} \mathrm{O}_{4}$ was the closest match to peak " 2 ," although several mixed iron 
manganese variations could have existed according to the High Score Plus database. From the data in Figure 6.5A, it appeared that peak "1" and "2" existed simultaneously during reduction which indicated that as oxygen from $\mathrm{CuFeMnO}_{4}$ was consumed a new phase was promoted with the remaining reactive oxygen. As the TPR progressed, peak " 2 " decreased in intensity and peaks " 3 ," “4," and "5" formed. TPR with $\mathrm{CH}_{4}$ was terminated above $900^{\circ} \mathrm{C}$ to avoid $\mathrm{CH}_{4}$ decomposition within the instrument and thus metallic manganese was not identified as indicated in Figure 6.5B. Peak " 3 " indicated the formation of $\mathrm{MnO}$. Peaks "4" and "5" were located approximately at the characteristic peaks for metallic copper, and metallic iron, respectively, which signified the continuous formation of reduced metal phases due to reduction. It is not believed that peak "4" and " 5 " were the result of the formation of additional metal oxide phases.

The combination of DSC/TGA and in-situ XRD data provided excellent insight into the reduction pathway of the trimetallic OC. Exothermic DSC data may correlate to the consumption of peak "1," and endothermic DSC data may correlate to the consumption of peak "2," which originated at $600{ }^{\circ} \mathrm{C}$. It is likely that the solid-solid phase transition of peak "1" to peak "2" contributed additional endothermic heat flow observed during DSC/TGA TPR with $\mathrm{CH}_{4}$ of $\mathrm{CuFeMnO}_{4}$. During the phase transition from peak "1" to peak "2," the XRD signal appeared broad and undefined, which could be caused by either lattice shrinking due to the formation of oxygen vacancies during reduction or multiple intermediate phases. The formation of a new oxygen depleted phase was apparent, however, by the distinct formation of peak B above $600{ }^{\circ} \mathrm{C}$.

Confirmation of the solid-solid phase transition provided a crucial piece of information to the reduction mechanism that allowed a series model to be adopted where the weight change during the exothermic portion is deconvoluted from the endothermic portion. Trimetallic $\mathrm{CuFeMnO}_{4}$ contributes only the initial portion of the entire reduction pathway. The secondary phase imitated 
a new reduction route which is most likely controlled by an entirely new mechanism. Ideally in a large-scale CLC reactor, the OC should only operate in the exothermic region so that limited heat transfer back to the fuel reactor is necessary to sustain auto-thermal operation. The combination of simultaneous DSC/TGA and in-situ XRD temperature programmed reductions indicated ideal operating oxygen transfer capacity of the trimetallic OC. 


\section{Chapter 7. Reaction Characterization and Kinetic Modeling during the Reduction}

of $\mathrm{CuFeMnO}_{4}$ with $\mathrm{H}_{2}$

\subsection{DSC/TGA during TPR with $\mathrm{H}_{2}$ Performance Data Comparison of $\mathrm{CuFeMnO}_{4}$ and} Corresponding Pure Metal Oxides

As shown by the experimental heat flow, weight change, and global reaction rate data in Figure 7.1A-C, respectively, DSC/TGA during TPR with $20 \% \mathrm{H}_{2}$ balance Ar was conducted to compare the reduction pathway of the trimetallic OC with that of the single metal counterparts.
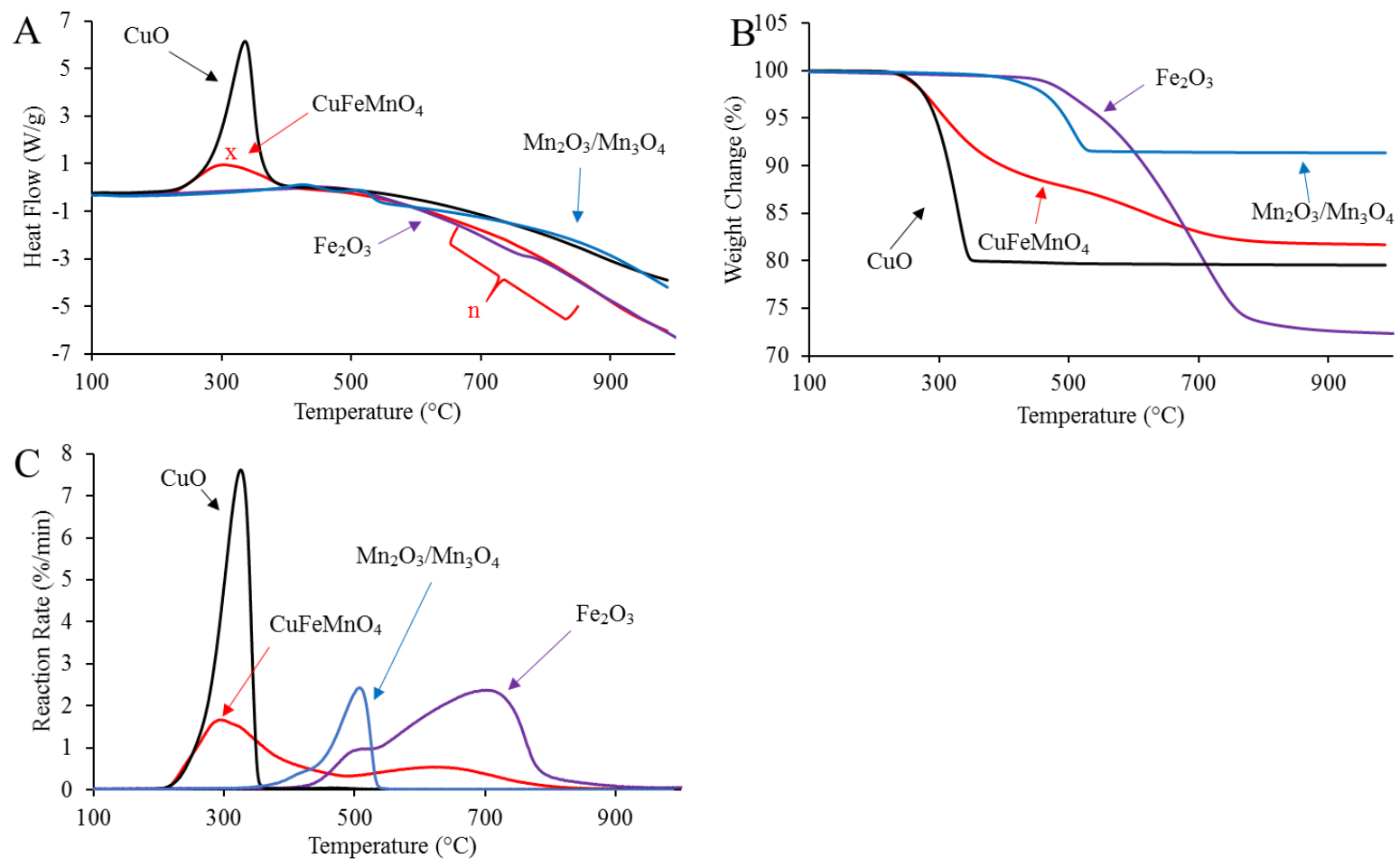

Figure 7.1 DSC/TGA during TPR with $20 \% \mathrm{H}_{2}$ balance Ar performance data comparison of $\mathrm{CuFeMnO} 4$ and single metal counterparts $\left(\mathrm{CuO}, \mathrm{Mn}_{2} \mathrm{O}_{3} / \mathrm{Mn}_{3} \mathrm{O}_{4}, \mathrm{Fe}_{2} \mathrm{O}_{3}\right)$

During the TPR with $\mathrm{H}_{2}$ and $\mathrm{CuFeMnO}_{4}$, two reactions were observed as indicated by changes in heat flow and global reaction rates as shown in red in Figure 7.1A and Figure 7.1B respectively. The first reaction was observed at $294.5^{\circ} \mathrm{C}$ as indicated by the first global reaction peak maximum at this temperature, shown in Figure 7.1A, and the corresponding exothermic heat flow data which is shown in Figure 7.1C. The exothermic and endothermic heat flow data during 
DSC/TGA TPR with $\mathrm{H}_{2}$ and $\mathrm{CuFeMnO}_{4}$ in Figure $7.1 \mathrm{~A}$ is labelled " $x$," and " $n$," to signify exothermic and endothermic data, respectively. The reaction rate data shown in Figure 7.1C is the differentiation with respect to time of the weight change profiles shown in Figure 7.1B. A second reaction was observed which occurred over a broader temperature range and lacked the magnitude in reaction rate maxima as compared to the initial exothermic reaction. The maximum of this global reaction rate occurred at $628.7{ }^{\circ} \mathrm{C}$ and corresponding endothermic data was observed. The exothermic and endothermic features observed from this reaction profile suggested a unique reaction network possibly due to a combination of metal oxide phases inherent to the trimetallic system, and this is explored further in the following sections. Experimentally observed exothermic heat flow during reduction suggested that auto-thermal operation with this OC could be more easily sustained.

As shown by the DSC/TGA experiments during TPR with $\mathrm{H}_{2}$ in Figure 7.1 , the TPR reaction data of $\mathrm{CuFeMnO}_{4}$ and $\mathrm{H}_{2}$ demonstrated unique heat flow and corresponding reaction rate maxima which were different from that with $\mathrm{CuO}, \mathrm{Fe}_{2} \mathrm{O}_{3}$, and $\mathrm{Mn}_{2} \mathrm{O}_{3} / \mathrm{Mn}_{3} \mathrm{O}_{4}$. During DSC/TGA TPR with $\mathrm{H}_{2}$ and $\mathrm{CuO}$, a global reaction rate maximum with corresponding exothermic heat flow can be observed at $325.7^{\circ} \mathrm{C}$. The reduction of $\mathrm{CuO} \rightarrow \mathrm{Cu}$ was rapid and was highly exothermic. Due to the proximity of the reaction rate maxima data between $\mathrm{CuFeMnO}_{4}$ and $\mathrm{CuO}$, it appeared that $\mathrm{H}_{2}$ preferentially reacted with oxygen bound to the $\mathrm{Cu}^{2+}$ cations in the trimetallic oxide. However, the distinct differences in intensity and profile of the heat flow data between $\mathrm{CuO}$ and $\mathrm{CuFeMnO}_{4}$ demonstrated that $\mathrm{CuO}$ did not represent a dominant phase in the trimetallic system. During DSC/TGA TPR with $\mathrm{H}_{2}$ and $\mathrm{Fe}_{2} \mathrm{O}_{3}$, a global reaction rate maximum was observed at $521.4^{\circ} \mathrm{C}$ with observed exothermic heat flow and corresponded to the reduction of $\mathrm{Fe}_{2} \mathrm{O}_{3} \rightarrow \mathrm{Fe}_{3} \mathrm{O}_{4}$. Following this reaction, a global reaction rate maximum was observed $701.2{ }^{\circ} \mathrm{C}$. This global 
reaction rate occurred over a broad temperature range and produced endothermic heat flow. The DSC/TGA during TPR data with $\mathrm{H}_{2}$ and pure $\mathrm{Fe}_{2} \mathrm{O}_{3}$ indicated that pure $\mathrm{Fe}_{2} \mathrm{O}_{3}$ was not a dominant phase in the trimetallic system. The DSC/TGA during TPR with $\mathrm{H}_{2}$ and $\mathrm{Mn}_{2} \mathrm{O}_{3} / \mathrm{Mn}_{3} \mathrm{O}_{4}$ data demonstrated a global reaction rate maximum had occurred at $508.0^{\circ} \mathrm{C}$ with observed exothermic heat flow. This reaction was due to the transition of $\mathrm{Mn}_{3} \mathrm{O}_{4} \rightarrow \mathrm{MnO}$. At the onset of this reaction additional exothermic heat was observed which correlated with the reduction of $\mathrm{Mn}_{2} \mathrm{O}_{3} \rightarrow \mathrm{MnO}$. Most likely, a mixed phase of $\mathrm{Mn}_{2} \mathrm{O}_{3} / \mathrm{Mn}_{3} \mathrm{O}_{4}$ phase had been formed after calcination, however, according to the DSC/TGA TPR data, $\mathrm{Mn}_{2} \mathrm{O}_{3} / \mathrm{Mn}_{3} \mathrm{O}_{4}$ did not represent a dominant phase in the trimetallic system.

Table 7.1. Theoretical enthalpy of reaction calculation with pure metal oxides and $\mathrm{H}_{2}$

\begin{tabular}{|c|c|}
\hline Reaction & $\Delta \mathrm{H}_{800}(\mathrm{KJ})$ \\
\hline $2 \mathrm{CuO}+\mathrm{H}_{2} \rightarrow \mathrm{Cu}_{2} \mathrm{O}+\mathrm{H}_{2} \mathrm{O}$ & -116.0 \\
\hline $\mathrm{Cu}_{2} \mathrm{O}+\mathrm{H}_{2} \rightarrow 2 \mathrm{Cu}+\mathrm{H}_{2} \mathrm{O}$ & -81.1 \\
\hline $3 \mathrm{Fe}_{2} \mathrm{O}_{3}+\mathrm{H}_{2} \rightarrow 2 \mathrm{Fe}_{3} \mathrm{O}_{4}+\mathrm{H}_{2} \mathrm{O}$ & -5.3 \\
\hline $\mathrm{Fe}_{3} \mathrm{O}_{4}+\mathrm{H}_{2} \rightarrow 3 \mathrm{FeO}+\mathrm{H}_{2} \mathrm{O}$ & +50.5 \\
\hline $\mathrm{FeO}+\mathrm{H}_{2} \rightarrow \mathrm{Fe}+\mathrm{H}_{2} \mathrm{O}$ & $+16.1 * \Delta \mathrm{G}_{800}>0$ \\
\hline $3 \mathrm{Mn}_{2} \mathrm{O}_{3}+\mathrm{H}_{2} \rightarrow 2 \mathrm{Mn}_{3} \mathrm{O}_{4}+\mathrm{H}_{2} \mathrm{O}$ & -176.1 \\
\hline $\mathrm{Mn}_{3} \mathrm{O}_{4}+\mathrm{H}_{2} \rightarrow 3 \mathrm{MnO}+\mathrm{H}_{2} \mathrm{O}$ & -19.7 \\
\hline $\mathrm{MnO}_{2}+\mathrm{H}_{2} \rightarrow \mathrm{Mn}+\mathrm{H}_{2} \mathrm{O}$ & $+137.6 * \Delta \mathrm{G}_{800}>0$ \\
\hline
\end{tabular}

The experimental heat flow data observed during DSC/TGA during TPR with $\mathrm{H}_{2}$ and the single metal oxides qualitatively agreed with theoretical enthalpy of reaction calculations using FactSage software shown in Table 7.1. It should be noted that the calculated Gibbs free energies of the reduction of $\mathrm{FeO} \rightarrow \mathrm{Fe}$ and $\mathrm{MnO} \rightarrow \mathrm{Mn}$ are positive and not favorable at $800{ }^{\circ} \mathrm{C}$. The reduction of $\mathrm{Mn}_{2} \mathrm{O}_{3} \rightarrow \mathrm{Mn}_{3} \mathrm{O}_{4}$ with $\mathrm{H}_{2}$ exhibited the most substantial theoretical exothermic enthalpy of reaction, followed by $\mathrm{CuO} \rightarrow \mathrm{Cu}_{2} \mathrm{O}, \mathrm{Cu}_{2} \mathrm{O} \rightarrow \mathrm{Cu}, \mathrm{Mn}_{3} \mathrm{O}_{4} \rightarrow \mathrm{MnO}$, and lastly $\mathrm{Fe}_{2} \mathrm{O}_{3} \rightarrow$ $\mathrm{Fe}_{3} \mathrm{O}_{4}$. The reduction of $\mathrm{Fe}_{3} \mathrm{O}_{4} \rightarrow \mathrm{FeO}$ demonstrated theoretical endothermic enthalpies of reaction. Given the ability of $\mathrm{CuO}$ to produce a large amount of heat during reduction with $\mathrm{H}_{2}$ it 
was apparent that reactions with oxygen bound to $\mathrm{Cu}$ in the trimetallic lattice would produce exothermic heat flow, but in the context of the trimetallic system, the observed exothermic heat flow was due to a reaction between $\mathrm{Cu}^{2+}$ and oxygen bound to the trimetallic lattice rather than pure unbound $\mathrm{CuO}$.

Table 7.2. Experimental and theoretical oxygen transfer capacities of pure metal oxides and $\mathrm{CuFeMnO}_{4}$

\begin{tabular}{|c|c|c|}
\hline Reaction & $\begin{array}{c}\text { Theoretical } \\
\text { Oxygen } \\
\text { Transfer }\end{array}$ & $\begin{array}{c}\text { Experimental } \\
\text { Oxygen Transfer }\end{array}$ \\
\hline $\mathrm{CuO}+\mathrm{Fe}_{3} \mathrm{O}_{4}+\mathrm{Mn}_{3} \mathrm{O}_{4}+6 \mathrm{H}_{2} \rightarrow \mathrm{Cu}+3 \mathrm{MnO}+3 \mathrm{Fe}+6 \mathrm{H}_{2} \mathrm{O}$ & $17.9 \%$ & $18.3 \%$ \\
\hline $\mathrm{CuFeMnO} \mathrm{O}_{4}+2,3 \mathrm{H}_{2} \rightarrow \mathrm{CuFeMnO} / \mathrm{CuFeMnO}+2,3 \mathrm{H}_{2} \mathrm{O}$ & $13.4 \%-20.1 \%$ & $18.3 \%$ \\
\hline $\mathrm{CuO}+\mathrm{H}_{2} \rightarrow \mathrm{Cu}+\mathrm{H}_{2} \mathrm{O}$ & $20.1 \%$ & $20.1 \%$ \\
\hline $\mathrm{Fe}_{2} \mathrm{O}_{3}+3 \mathrm{H}_{2} \rightarrow 2 \mathrm{Fe}+3 \mathrm{H}_{2} \mathrm{O}$ & $30.1 \%$ & $28.0 \%$ \\
\hline $\mathrm{Mn}_{2} \mathrm{O}_{3}+\mathrm{H}_{2} \rightarrow 2 \mathrm{MnO}+\mathrm{H}_{2} \mathrm{O}$ & $10.1 \%$ & $8.6 \%$ \\
\hline
\end{tabular}

As shown by the data in Table 7.2, the highest oxygen transfer capacity was achieved by $\mathrm{Fe}_{2} \mathrm{O}_{3}$, but the reaction rate maximum occurred at much elevated temperatures from that of the other single metal oxides and $\mathrm{CuFeMnO}_{4}$. Despite the large oxygen transfer capacity, reduction of $\mathrm{Fe}_{2} \mathrm{O}_{3}$ to $\mathrm{Fe}$ was mostly endothermic and displayed slow reaction rates as shown in Figure 7.1C. The reduction of trimetallic $\mathrm{CuFeMnO}_{4}$ initiated at the lowest temperature and demonstrated improved heat flow and reaction rate data than that with $\mathrm{Fe}_{2} \mathrm{O}_{3}$ or $\mathrm{Mn}_{2} \mathrm{O}_{3}$. Although $\mathrm{CuO}$ displayed high oxygen transfer capacity and significant exothermic heat flow, agglomeration and attrition issues plague this metal oxide [35]. Incorporation of $\mathrm{Fe}_{2} \mathrm{O}_{3}$ and $\mathrm{Mn}_{2} \mathrm{O}_{3}$ with $\mathrm{CuO}$ maintained high oxygen transfer capacity, exothermic heat flow, and reaction rate data during reduction. 
7.2. DSC/TGA during TPR with $\mathrm{H}_{2}$ Performance Data Comparison of $\mathrm{CuFeMnO}_{4}$ and Corresponding Bimetallic Oxides

As shown by the experimental heat flow, weight change, and global reaction rate data in Figure 7.2A-C respectively, DSC/TGA during TPR with $20 \% \mathrm{H}_{2}$ balance Ar was conducted to compare the reduction pathway of the trimetallic OC with that of the bimetallic counterparts.
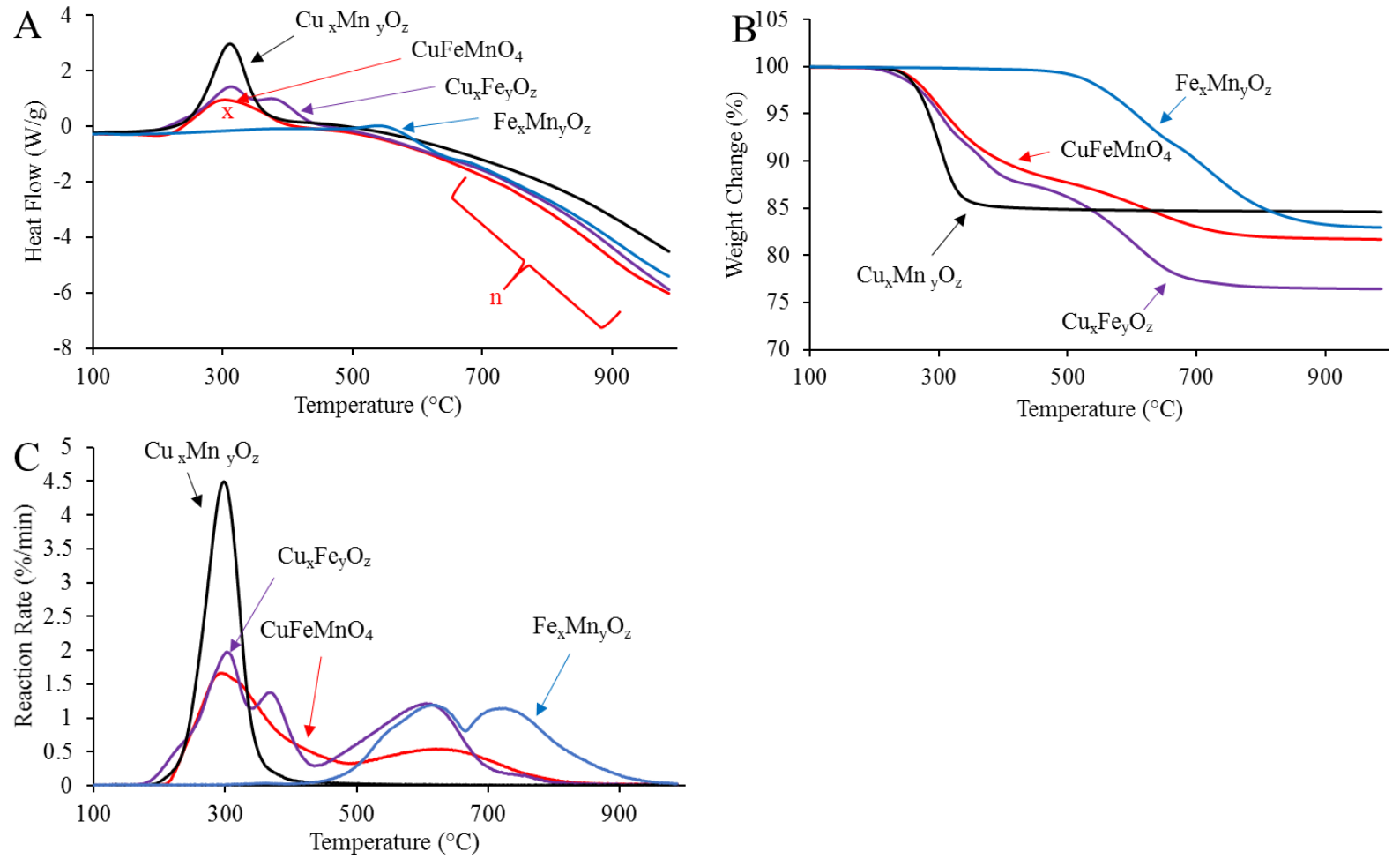

Figure 7.2. DSC/TGA during TPR with $20 \% \mathrm{H}_{2}$ balance Ar performance data comparison of $\mathrm{CuFeMnO}_{4}$ and bimetallic counterparts

As shown by the DSC/TGA performance data of $\mathrm{CuFeMnO}_{4}$ and corresponding bimetallic counterparts during TPR with $\mathrm{H}_{2}$ in Figure 7.2, the $\mathrm{Cu}$ containing materials demonstrated a low temperature exothermic reaction. It was apparent from the DSC/TGA performance data that the presence of copper enhances the low temperature exothermic portion of reduction. However, differences in magnitude and profile from pure $\mathrm{CuO}$ indicated that this low temperature reduction enhancement was caused by the formation of complex trimetallic species and not simply due to 
dissociated $\mathrm{CuO}$. During the reduction of copper manganese oxide, a global reaction rate maximum is observed at $298.2{ }^{\circ} \mathrm{C}$ with a corresponding exothermic heat flow. Incorporation of copper and manganese clearly improved the reactivity and promoted an exothermic reduction reaction, however, differences in the intensity of the heat flow and reaction rate data indicated that copper manganese oxide did not represent a dominant phase in the trimetallic system. Bimetallic copper iron oxide demonstrated two reaction rate maxima at $302.2{ }^{\circ} \mathrm{C}$ and $367.9{ }^{\circ} \mathrm{C}$ with corresponding exothermic heat flow data and at a reaction rate maximum at $605.9{ }^{\circ} \mathrm{C}$ that corresponded with endothermic heat flow. The presence of the exothermic doublet with copper iron oxide indicated that reduction in this system was due to multiple phases at low temperature and furthermore differences in reaction rate maxima during the endothermic portion of reduction at elevated temperatures indicated that copper iron oxide did not represent a dominant phase in the trimetallic system.

During DSC/TGA TPR with $\mathrm{H}_{2}$ and iron manganese oxide, exothermic heat flow data can be observed upon the onset of the global reaction rate maximum at $620.9^{\circ} \mathrm{C}$. Endothermic heat flow was observed at the reaction rate maximum and additional endothermic heat flow was observed at the second reaction rate maxima at $721.9^{\circ} \mathrm{C}$. Due to the differences in observed heat flow and reaction rate maxima data, bimetallic iron manganese oxide did not represent a dominant phase in the trimetallic system. 
Table 7.3. Experimental and theoretical oxygen transfer capacities of bimetallic oxides and $\mathrm{CuFeMnO}_{4}$

\begin{tabular}{|c|c|c|}
\hline Reaction & $\begin{array}{c}\text { Theoretical } \\
\text { Oxygen Transfer }\end{array}$ & $\begin{array}{c}\text { Experimental } \\
\text { Oxygen Transfer }\end{array}$ \\
\hline $\mathrm{CuO}+\mathrm{Fe}_{3} \mathrm{O}_{4}+\mathrm{Mn}_{3} \mathrm{O}_{4}+3 \mathrm{H}_{2} \rightarrow \mathrm{Cu}+3 \mathrm{Fe}+3 \mathrm{MnO}+3 \mathrm{H}_{2} \mathrm{O}$ & $17.9 \%$ & $18.3 \%$ \\
\hline $\mathrm{CuFeMnO} \mathrm{O}_{4}+1,2 \mathrm{H}_{2} \rightarrow \mathrm{CuFeMnO} / \mathrm{CuFeMnO}+1,2 \mathrm{H}_{2} \mathrm{O}$ & $13.4 \%-20.1 \%$ & $18.3 \%$ \\
\hline $\mathrm{CuO}+\mathrm{Fe}_{2} \mathrm{O}_{3}+2,3 \mathrm{H}_{2} \rightarrow \mathrm{Cu}+\mathrm{FeO} / \mathrm{Fe}+2,3 \mathrm{H}_{2} \mathrm{O}$ & $15.1 \%-25.1 \%$ & $23.6 \%$ \\
\hline $\mathrm{CuO}+\mathrm{Mn}_{2} \mathrm{O}_{3}+2 \mathrm{H}_{2} \rightarrow \mathrm{Cu}+2 \mathrm{MnO}+\mathrm{H}_{2} \mathrm{O}$ & $15.1 \%$ & $15.4 \%$ \\
\hline $\mathrm{Fe}_{2} \mathrm{O}_{3}+\mathrm{Mn}_{2} \mathrm{O}_{3}+\mathrm{H}_{2} \rightarrow \mathrm{FeO} / \mathrm{Fe}+2 \mathrm{MnO}+2,3 \mathrm{H}_{2} \mathrm{O}$ & $10.1 \%-20.0 \%$ & $17.0 \%$ \\
\hline
\end{tabular}

According to the data shown in Table 7.3, all bimetallic oxides and $\mathrm{CuFeMnO}_{4}$ yielded very high oxygen transfer capacities for reactions involving $\mathrm{H}_{2}$. Bimetallic copper iron oxide demonstrated the highest oxygen transfer capacity during performance analysis of complex metal oxide systems. Compared to our previous studies with $\mathrm{CH}_{4}$, the reactions with $\mathrm{H}_{2}$ demonstrated rate maxima at significantly lower temperatures and heat flow data that differed in profile and magnitude [73]. Not only did this performance analysis highlight that the metal oxide phase played a vital role in observed reactivity but also the choice of reaction gas altered reduction pathways as well. 


\subsection{In-situ XRD TPR with $5 \% \mathrm{H}_{2}$ Analysis}

To further evaluate the observed chemical changes that had occurred during the unique reaction pathway of $\mathrm{CuFeMnO}_{4}$ with $\mathrm{H}_{2}$, in-situ XRD during TPR with $\mathrm{H}_{2}$ was conducted to correlate the observed change in reactivity with any structural changes to the lattice.

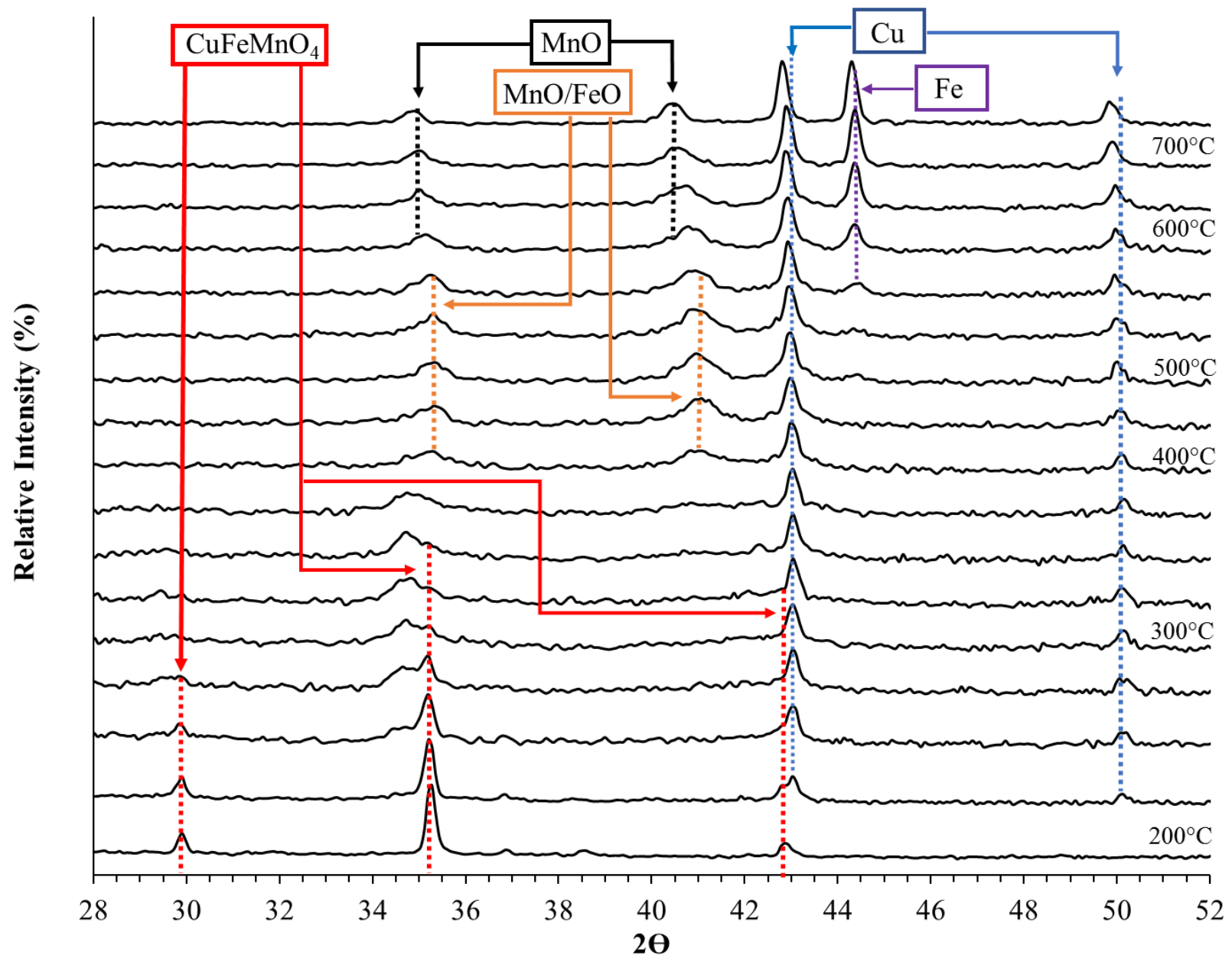

Figure 7.3 In-situ XRD of $\mathrm{CuFeMnO}_{4}$ during TPR with 5\% $\mathrm{H}_{2}$

According to the data in Figure 7.3, the trimetallic phase was initially detected due to the observation of the characteristic peak of $\mathrm{CuFeMnO}_{4}$ at $35.4952 \Theta\left(\begin{array}{lll}3 & 1 & 1\end{array}\right)$ at temperatures below $200{ }^{\circ} \mathrm{C}$. When the reaction initiated at approximately $200{ }^{\circ} \mathrm{C}$, the characteristic peak of $\mathrm{CuFeMnO}_{4}$ shifted left and broadened indicating lattice expansion and a decrease in grain size most likely due to the creation of oxygen vacancies from exposure to $\mathrm{H}_{2}$. Simultaneously, the formation of metallic 
$\mathrm{Cu}$ was detected. At $400{ }^{\circ} \mathrm{C}$, the peaks corresponding to the trimetallic phase completely disappeared and the formation of mixed $\mathrm{FeO}-\mathrm{MnO}$ phase was observed which indicated that oxygen bound in trimetallic phase was consumed during reactions with $\mathrm{H}_{2}$ and a new phase with new reaction properties had formed. At approximately $650{ }^{\circ} \mathrm{C}$, metallic $\mathrm{Fe}$ and $\mathrm{MnO}$ were observed simultaneously as the peaks of the mixed $\mathrm{FeO}-\mathrm{MnO}$ phase correspondingly disappeared. The apparent consumption of the $\mathrm{FeO}-\mathrm{MnO}$ phase to form $\mathrm{MnO}$ and $\mathrm{Fe}$ was most likely due creation of oxygen vacancies from reactions occurring with oxygen bound to $\mathrm{Fe}$ and $\mathrm{H}_{2}$. It appeared that $\mathrm{H}_{2}$ preferentially reacted with the cations distributed to the lattice of the trimetallic phase. $\mathrm{H}_{2}$ reacted with oxygen from $\mathrm{Cu}^{2+}$ in the lattice of $\mathrm{CuFeMnO}_{4}$ initially, followed by a reaction with oxygen from $\mathrm{Cu}$-depleted $\mathrm{Mn}^{3+}$ and $\mathrm{Fe}^{3+}$ phase which contributed to the formation of the mixed $\mathrm{FeO}-\mathrm{MnO}$ phase. These initial reactions could also be associated with the exothermic reactions that were observed during DSC/TGA analysis. Thus, the endothermic reaction could be attributed to the formation of metallic Fe from the mixed $\mathrm{FeO}-\mathrm{MnO}$ phase at deeper states of reduction. It appeared the mixed $\mathrm{FeO}-\mathrm{MnO}$ phase aided in the formation of metallic Fe during reduction since theoretical FactSage calculations suggested this was not favorable with pure iron oxides. In-situ XRD during TPR with $\mathrm{H}_{2}$ identified the presence of multiple metal oxide phases with unique reaction properties contributed to the reduction pathway of $\mathrm{CuFeMnO}_{4}$. In-situ XRD during TPR with $\mathrm{H}_{2}$ analysis further indicated that changes to the crystallite size due to peak broadening were indicative of oxygen exhaustion. This suggested that crystallite size may be affecting kinetic behavior and will be explored further during particle-scale kinetic modeling. 
7.4. In-situ XRD Temperature Programmed Oxidation (TPO) with 100\% Air Analysis As shown by the data in Figure 7.4, in-situ XRD during TPO of fully reduced $\mathrm{CuFeMnO}_{4}$ with air was conducted to confirm that the trimetallic phase could be regenerated during the cyclic CLC reactions.

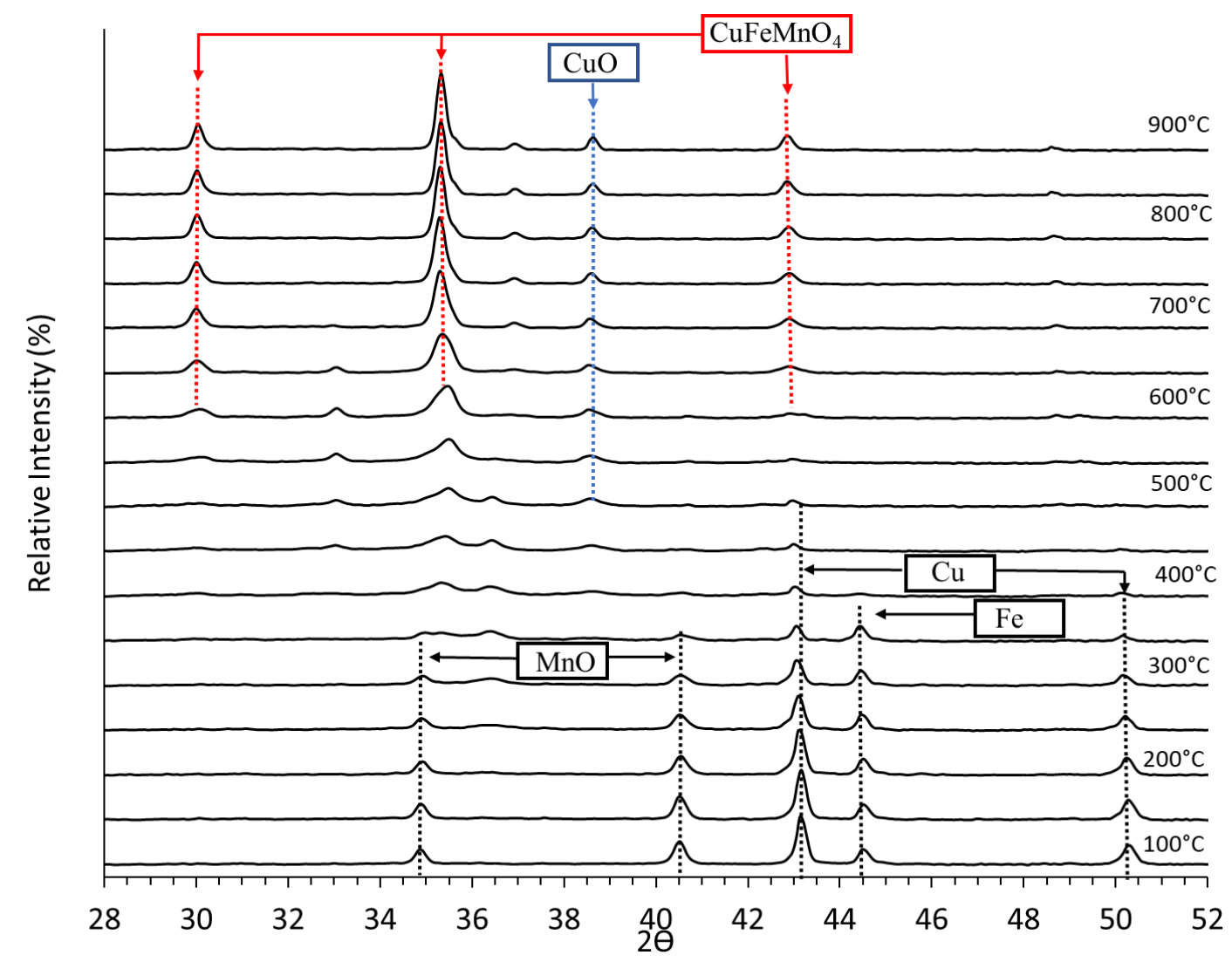

Figure 7.4 In-situ XRD of $\mathrm{CuFeMnO}_{4}$ during TPO with $100 \%$ air

According to the data in Figure 7.4, XRD peaks corresponding to metallic Fe and $\mathrm{MnO}$ disappeared around $400^{\circ} \mathrm{C}$ and the peaks from metallic $\mathrm{Cu}$ disappeared around $550{ }^{\circ} \mathrm{C}$. Between $400{ }^{\circ} \mathrm{C}$ and $500{ }^{\circ} \mathrm{C}$ only very low intensity XRD peaks were observed possibly due to formation of amorphous phases. However, around $650{ }^{\circ} \mathrm{C}$ the characteristic $\mathrm{XRD}$ peaks of $\mathrm{CuFeMnO}_{4}$ began to appear which indicated that the trimetallic phase was regenerable at temperatures exceeding 650 ${ }^{\circ} \mathrm{C}$. 


\subsection{Isothermal in-situ XRD with Controlled $\mathrm{H}_{2}$ Exposures at $800{ }^{\circ} \mathrm{C}$}

As shown in Figure 7.5, isothermal in-situ XRD experiments with controlled $\mathrm{H} 2$ exposures at $800{ }^{\circ} \mathrm{C}$ were conducted by incrementally dosing the sample with $5 \mathrm{~mL} \mathrm{H}_{2}$ injections and obtaining an XRD diffractogram after each injection. Isothermal conditions would be indicative of ideal CLC operation in a reducing reactor.

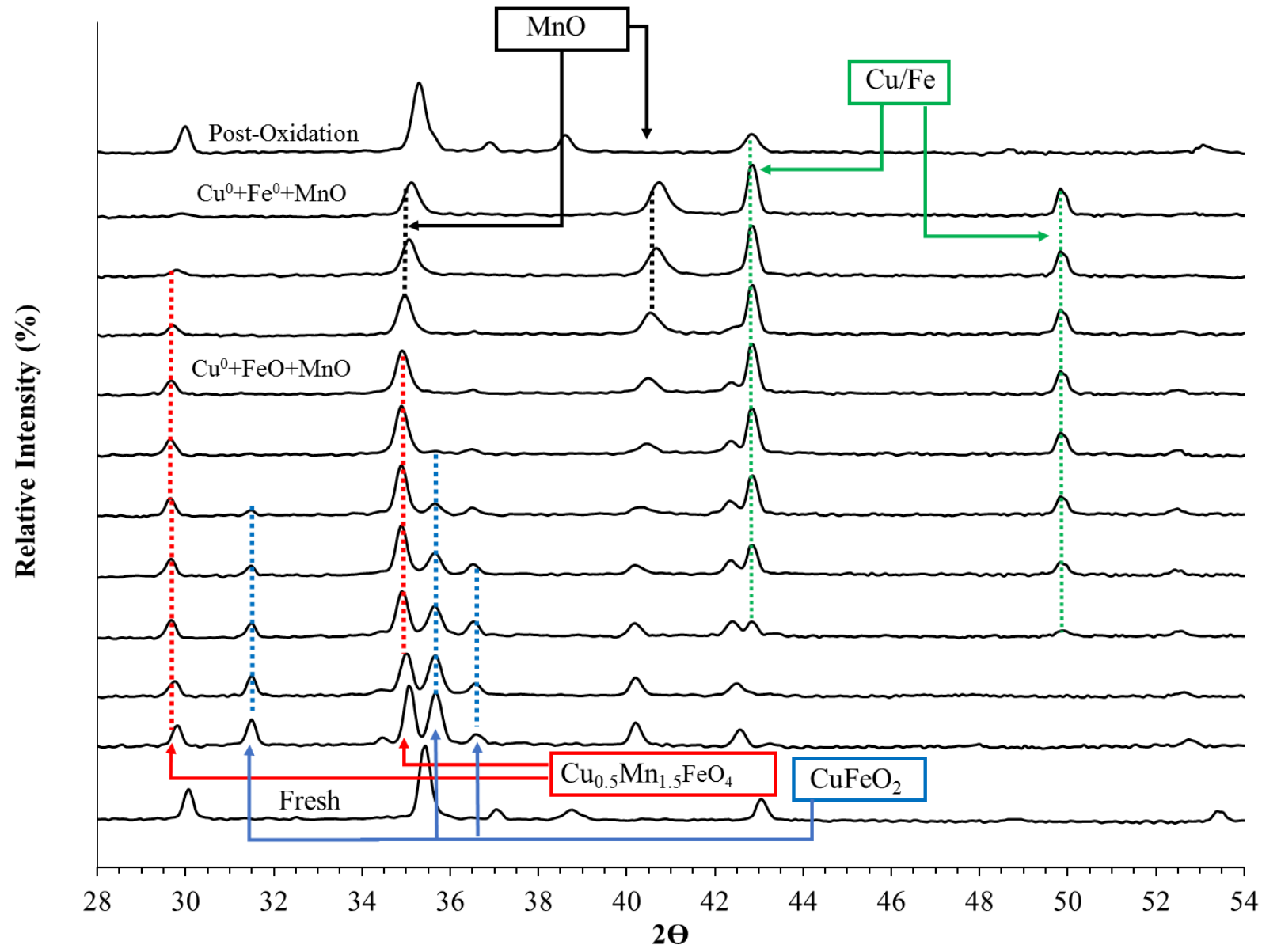

Figure 7.5. Isothermal in-situ XRD data during $\mathrm{H}_{2}$ doses at $800{ }^{\circ} \mathrm{C}$

According to the data in Figure 7.5, a bimetallic copper iron-rich phase and a manganeserich trimetallic phase and were detected after oxygen uncoupling in an inert environment at 800 ${ }^{\circ} \mathrm{C}$, prior to $\mathrm{H}_{2}$ introduction. As incremental $\mathrm{H}_{2}$ dosing began, the bimetallic copper iron-rich phase demonstrated immediate and significant peak broadening and some peak shifting to the left compared to that of the manganese rich trimetallic phase. After the fifth $\mathrm{H}_{2}$ dose $(\sim 10 \%$ 
oxygen transfer capacity), the bimetallic copper iron-rich phase was no longer detected, however, the manganese-rich trimetallic phase continued to exhibit peak shifting, broadening, and depletion. It was apparent that the oxygen from the manganese rich trimetallic phase had been exhausted of oxygen by the ninth injection ( $18 \%$ oxygen transfer capacity) which agreed with the theoretically calculated amount of oxygen available in the trimetallic system which was further evidenced by the detection of $\mathrm{MnO}, \mathrm{Cu}$, and $\mathrm{Fe}$ phases. From the previous discussion in Table 7.1, reduction of $\mathrm{MnO}$ is not favorable with $\mathrm{H}_{2}$ at $800{ }^{\circ} \mathrm{C}$. Once again crystallite size appeared to correlate with oxygen exhaustion and crystallite sizes were estimated with the Scherrer equation shown in Equation 7.1 where $\lambda,\left(\beta-\beta_{0}\right), \operatorname{Cos} \theta$, and $L$ represented the wavelength of copper $(1.54 \AA$ ), the full-width half maximum at the characteristic peak, the incident angle, and the crystallite diameter respectively [78]. The approximate grain size of $\mathrm{Cu}_{0.5} \mathrm{Mn}_{1.5} \mathrm{FeO}_{4}$ was estimated to be $86 \mathrm{~nm}$ and that of $\mathrm{CuFeO}_{2}$ was $209 \mathrm{~nm}$. The detection of two unique phase was confirmed with isothermal in-situ $\mathrm{XRD}_{2}$ exposures and crystallite sizes were determine which will be useful during the discussion of reaction modeling in the following sections.

$$
L=\frac{\lambda}{\left(\beta-\beta_{0}\right) \operatorname{Cos} \theta}
$$




\subsection{SEM-EDS Elemental Mapping}

SEM imaging and elemental mapping was conducted to observe features on the surface and cross-sectionally of the pellet that would help provide additional information in determining a reaction model.

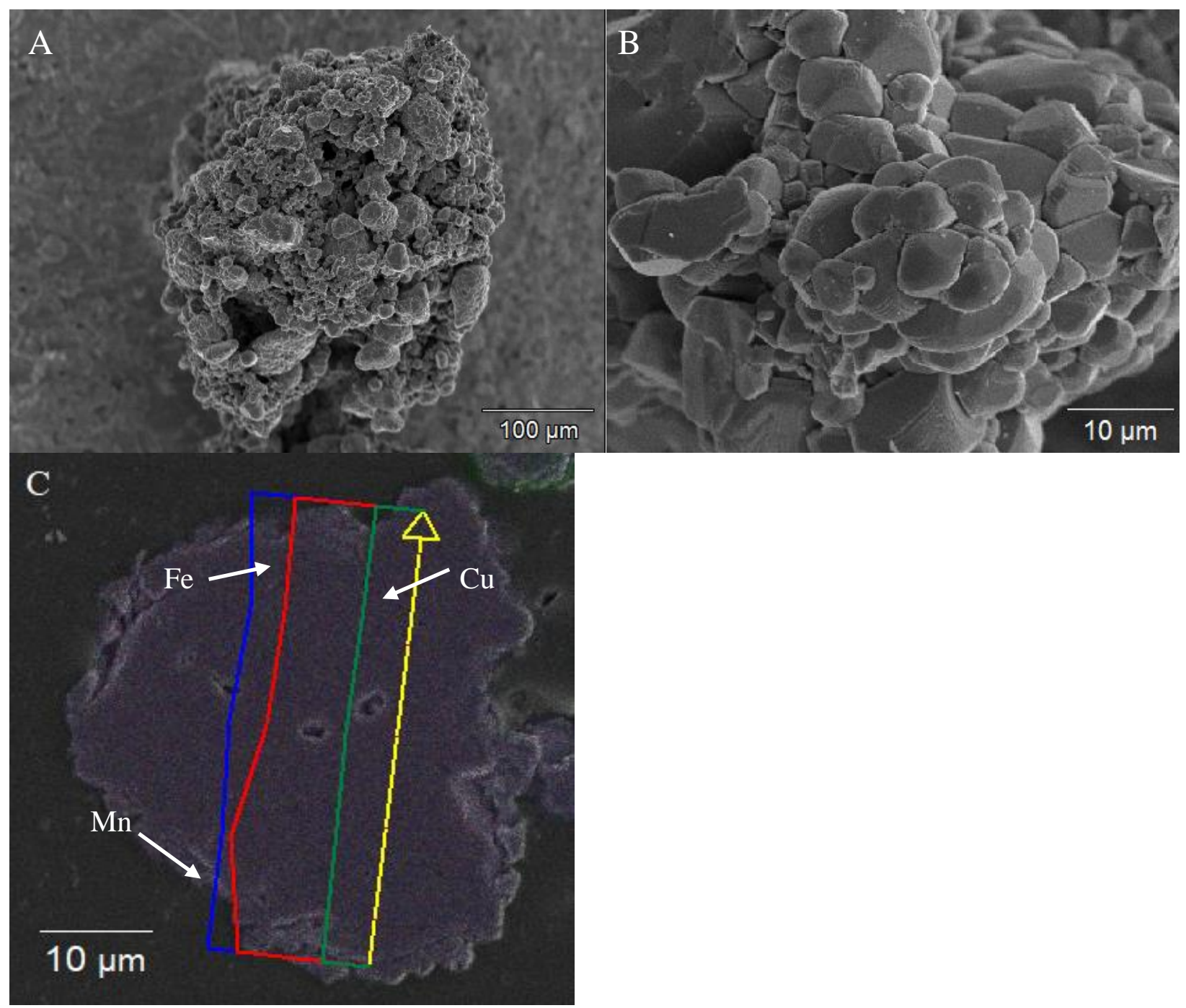

Figure 7.6. Fresh $\mathrm{CuFeMnO}_{4} \mathrm{SEM}-\mathrm{EDS}$ of $\mathrm{Cu}, \mathrm{Fe}$, and $\mathrm{Mn}$ are labeled green, red, and blue respectively (A) SEM exterior imaging (B) SEM cross-section imaging (C) EDS elemental mapping and line scan

According to the SEM image and cross-sectional EDS mapping data of a fresh pellet in Figure 7.6, a pellet composed of smaller micro-pellets with a well dispersed distribution of the metal cations was formed after calcination. As shown in Figure 7.6A, a macro-porous exterior can 
be observed and the bulk pellet appeared to be the result of the sintering of smaller granules as shown in Figure 7.6B with an average observed size of $5 \mu \mathrm{m}$. Cross-sectional imaging and EDS elemental data shown in Figure 7.6C indicated that the interior of the pellets was dense and a homogenous trimetallic phase had formed after calcination. As shown in Figure 7.6C, even distribution of $\mathrm{Cu}, \mathrm{Fe}$, and $\mathrm{Mn}$ represented by green, red, and blue respectively, indicated the consistency of the trimetallic phase throughout the pellet. 


\subsection{Isothermal Reduction Model Analysis of $\mathrm{CuFeMnO}_{4}$ with $\mathrm{H}_{2}$}

Before reaction parameters could be calculated from experimental TGA conversion-time data, reaction gas concentration, sample mass, reactor flow rate, and particle size were optimized so that rate-limiting effects from internal and external diffusional resistances would not affect the analysis as shown in Figure 7.7.
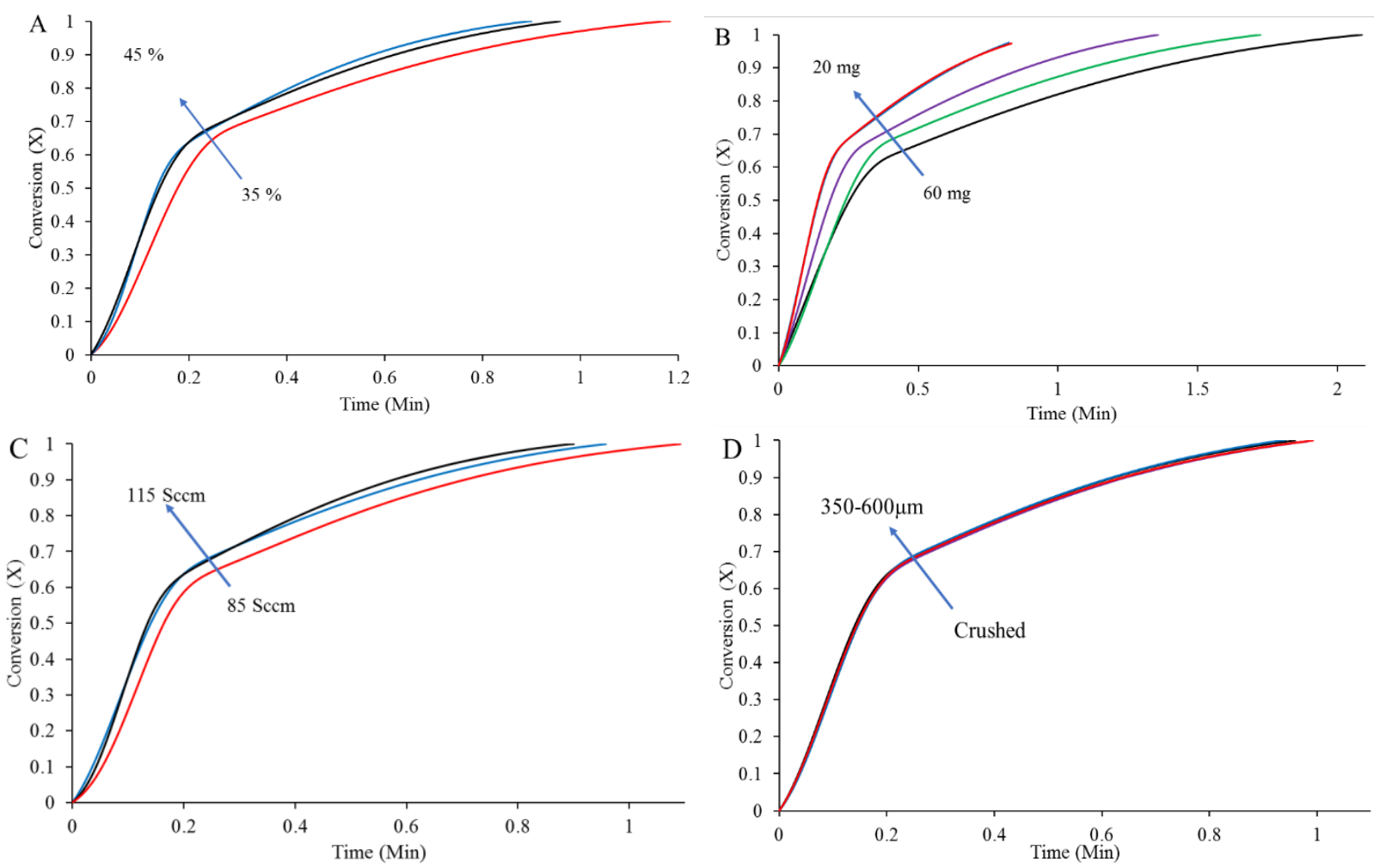

Figure 7.7. Reaction optimization during isothermal TGA $\mathrm{H}_{2}$ reductions at $800{ }^{\circ} \mathrm{C}$ with respect to varying (A) Concentration (B) Mass (C) Flow rate (D) Particle size

According to the data in Figure 7.7A, $25 \mathrm{mg}$ of the trimetallic oxygen carrier was exposed to various $\mathrm{H}_{2}$ volumetric concentrations at $100 \mathrm{SCCM}$ and $40 \mathrm{vol} \% \mathrm{H}_{2}$ was determined to be the optimal concentration for $\mathrm{H}_{2}$ reaction analysis. Exposing the pellets to concentrations below this value demonstrated slower observed conversion data and concentrations above this value did not influence on the observed conversion data. As shown by the data in Figure 7.7B, sample mass was optimized by exposing various sample masses to $40 \mathrm{vol} \% \mathrm{H}_{2}$ at $100 \mathrm{SCCM}$. The reactivity of the 
trimetallic oxygen carrier was unaffected by loading the TGA reactor with sample masses below $25 \mathrm{mg}$. As demonstrated by the data in Figure 7.7C, flow rate was optimized by exposing $25 \mathrm{mg}$ of $\mathrm{CuFeMnO}_{4}$ with 40 vol\% $\mathrm{H}_{2}$ at various flow rates. Above $100 \mathrm{SCCM}$, external diffusional limitations in the boundary layer could be neglected. According to the data in Figure 7.7D, the effect of particle size was evaluated by exposing $25 \mathrm{mg}$ of $\mathrm{CuFeMnO}_{4}$ to $40 \mathrm{vol}^{2} \mathrm{H}_{2}$ at $100 \mathrm{SCCM}^{-}$ in ranges of $600-350 \mu \mathrm{m}, 250-350 \mu \mathrm{m}, 180-250 \mu \mathrm{m}$, and crushed $(<74 \mu \mathrm{m})$. There was no observed change in reactivity when particle size was varied which indicated that the gas diffusion within the pellet was fast and not the rate controlling step. As suggested by XRD analysis shown previously, the grain crystallite size appeared to be the controlling surface and chemical and diffusional resistance through the crystallites will be analyzed next. 

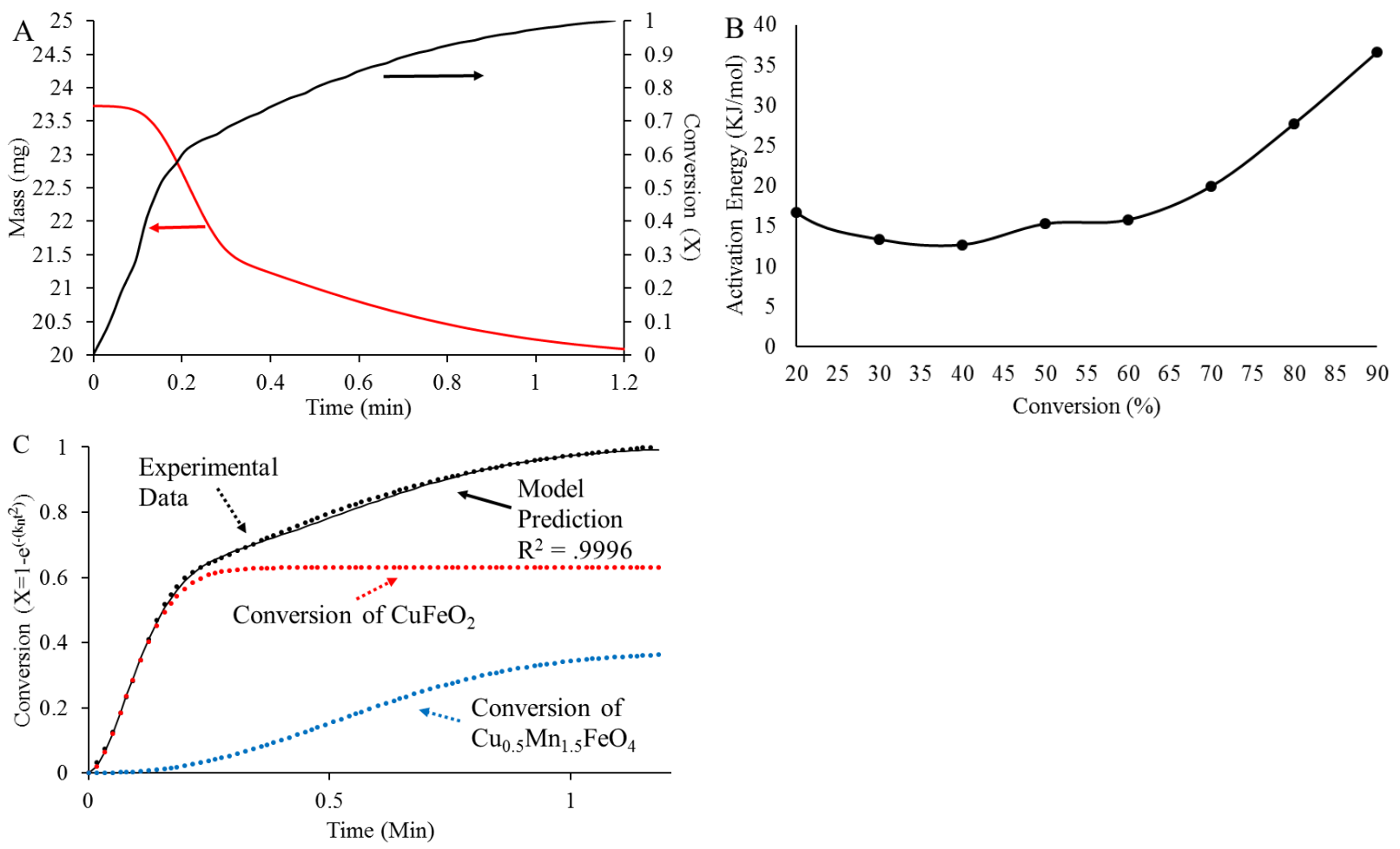

Figure 7.8. (A) TGA reduction of $\sim 25 \mathrm{mg} \mathrm{CuFeMnO} 4$ with $40 \% \mathrm{H}_{2}$ at $800^{\circ} \mathrm{C}$ and (B) Model-free Fitting (C) Deconvolution of phases

An example of a typical TGA weight change and conversion profile is shown in Figure 7.8A. As shown by isothermal experimental data in Figure $7.8 \mathrm{~A}$, a rapid reaction occurred at $800^{\circ} \mathrm{C}$ initially with $40 \mathrm{vol} \% \mathrm{H}_{2}$ as evidenced by the observed substantial weight change. Following this reaction, the observed reactivity appeared to decrease as demonstrated by the slower observed rates sustained during the last portion of the reaction. Conversion was calculated using the experimental weight loss data from Figure 7.8A in Equation 4.1.

To gain a deeper understanding of the reduction pathway of $\mathrm{CuFeMnO}_{4}$ with respect to $\mathrm{H}_{2}$, model-free analysis with conversion data collected at $900{ }^{\circ} \mathrm{C}, 850{ }^{\circ} \mathrm{C}, 800{ }^{\circ} \mathrm{C}, 750{ }^{\circ} \mathrm{C}$, and $700{ }^{\circ} \mathrm{C}$ was conducted as shown in Figure 7.8B. Model-free analysis removes the assumption of a reaction mechanism as shown in Equation 4.24 and values provided by this analysis also allow for comparison with parameters derived from reaction models where a mechanism has been assumed [79]. 
According to model-free analysis, the observed activation energy remained low around $14 \mathrm{KJ} / \mathrm{mol}$ during the initial $60 \%$ of conversion, following this initial low activation energy regime, a steady rise in activation energy can be observed with a corresponding value approaching $\sim 40 \mathrm{KJ} / \mathrm{mol}$ towards the completion of the reaction. From this analysis, it was apparent that the two observed phases from in-situ XRD analysis shown in the previous section have different reaction properties which significantly influence the varying rates and activation energies of the reaction profile of $\mathrm{CuFeMnO}_{4}$ with $\mathrm{H}_{2}$ and contribute to the change in activation energy. Various reaction models in Table 4.1 were examined to fit the conversion data from the TGA experiments and it was found that nucleation and growth kinetics demonstrated the best representation of the characterization data and reaction data.

In order to deconvolute the contributions from the two independent reaction shown in Equation 7.2 and Equation 7.3, a parallel approach of nucleation and growth models shown in Equation 7.4 was used to represent the two independent reactions a serve as the framework for the overall reaction. As shown in Equation 7.4, the summation of the converted oxygen from the individual phases yielded the total oxygen depleted from the trimetallic oxide.

$$
\begin{gathered}
\mathrm{CuFeO}_{2}+2 \mathrm{H}_{2} \rightarrow \mathrm{Cu}+\mathrm{Fe}+2 \mathrm{H}_{2} \mathrm{O} \\
\mathrm{Cu}_{0.5} \mathrm{Mn}_{1.5} \mathrm{FeO}_{4}+2.5 \mathrm{H}_{2} \rightarrow 0.5 \mathrm{Cu}+\mathrm{Fe}+1.5 \mathrm{MnO}+2.5 \mathrm{H}_{2} \mathrm{O} \\
X=\varphi_{A}\left(1-e^{-k_{A n} t^{n_{A}}}\right)+\varphi_{B}\left(1-e^{-k_{B_{n}} t^{n_{B}}}\right)
\end{gathered}
$$

In Equation 7.4, $\mathrm{X}, \varphi_{i}, \mathrm{k}_{\mathrm{n}}$, and $\mathrm{n}$ represented, conversion, oxygen density, nucleation growth rate constant, and the nucleation constant for Phase $\mathrm{A} \mathrm{CuFeO}_{2}$, and Phase $\mathrm{B}$ $\mathrm{Cu}_{0.5} \mathrm{Mn}_{1.5} \mathrm{FeO}_{4}$, respectively. It should be noted that the phases of $\mathrm{CuFeO}_{2}$ and $\mathrm{Cu}_{0.5} \mathrm{Mn}_{1.5} \mathrm{FeO}_{4}$ were identified during isothermal in-situ XRD analysis previously shown in Figure 7.5. For 
reaction modeling, oxygen density was predetermined from the theoretical oxygen transfer capacities of the two phases compared to the total observed oxygen transfer capacity. For $\mathrm{CuFeO}_{2}$, oxygen density was fixed to $60 \%$ and $\mathrm{Cu}_{0.5} \mathrm{Mn}_{1.5} \mathrm{FeO}_{4}$ at $40 \%$ which also agreed with Rietveld analysis performed during isothermal XRD analysis. The theoretically calculated densities agreed well with the change in activation energy observed in model-free analysis. The nucleation constant was also held constant at 2 which both models favored during the initial reaction analysis shown in Figure 7.8 and is indicative of "plate-like" geometry which agreed with the 1-demnsional (1-D) surface reaction controlled mechanisms to be performed next [61].

As shown in Figure $7.8 \mathrm{C}$, both phases exhibited a strong fit to nucleation and growth kinetics and two reaction profiles were found using Equation 7.4. Deconvoluting the phases isolated the metal oxide phase contributing to the reaction thus eliminating the misrepresented kinetics from a combination of phases. With two independent reaction profiles, 1-D grain boundary reaction control and 1-D diffusional control models were utilized to calculate reaction and effective diffusion coefficients as shown in Equations 7.5 and 7.6. in Figure 7.9 [80]. This type of system has been described previously as the grainy pellet model where the reactions that occur in the grain are represented of conversion throughout the bulk of the pellet [57]. 1-D boundary models were employed to provide reaction parameters with units that are agreed upon in literature. These calculated constants from 1-D boundary analysis were then tested with established dimensionless mass transport parameters to confirm the intrinsic nature of the values obtained from model analysis [81]. These parameters with accepted units can then be inserted into the framework of the nucleation and growth mechanism that serves as the overarching backbone in the model. 

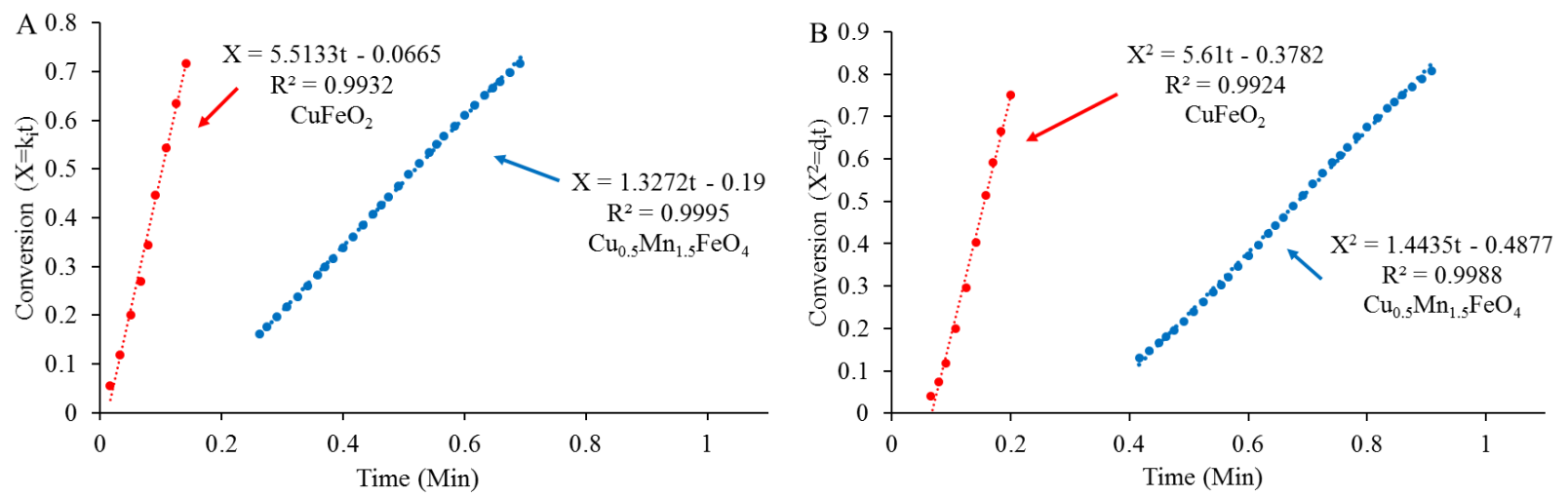

Figure 7.9. Determination of rate constants from (A) 1-D grain boundary and (B) 1-D diffusional reaction models during TGA $40 \% \mathrm{H}_{2}$ Reduction at $800{ }^{\circ} \mathrm{C}$

$$
\begin{gathered}
X=k_{i} t \text { where } k_{i}=\frac{b k_{S} C_{H_{2}}}{L \rho} \\
X^{2}=d_{i} t \text { where } d_{i}=\frac{b D_{e} C_{H_{2}}}{R^{2} \rho}
\end{gathered}
$$

In Equations 7.5 and 7.6, $\mathrm{X}, \mathrm{k}_{\mathrm{i}}, \mathrm{b}, \mathrm{k}_{\mathrm{s}}, C_{\mathrm{H}_{2}}, \rho, \mathrm{d}_{\mathrm{i}}, \mathrm{D} \mathrm{e}, \mathrm{L}$, and $\mathrm{R}$ represented conversion, reaction time constant, stoichiometric ratio, reaction rate constant, $\mathrm{H}_{2}$ concentration, bulk density, diffusion time constant, effective diffusion, characteristic crystallite length, and characteristic granule radius respectively. Helium pycnometry, the Scherrer equation from XRD analysis, and SEM imaging were used to determine the values of bulk density, crystallite length and granule radius respectively, and each phase exhibited excellent linear fit with 1-D surface chemical and diffusional analysis. The values shown in Table 7.4 and obtained from 1-D model analysis were then tested with dimensionless mass transport analysis to confirm the values obtained from the experimental set up were not hindered by diffusional limitations in the bulk or surface of the grain or pellet. 
Table 7.4. Reaction constants obtained from 1-D model analysis (A) $\mathrm{CuFeO}_{2}$ (B) $\mathrm{Cu}_{0.5} \mathrm{Mn}_{1.5} \mathrm{FeO}_{4}$

\begin{tabular}{|c|c|}
\hline Parameter & Value (*Measured from Figure 7.9) \\
\hline $\mathrm{k}_{\mathrm{s}}$ & $* \mathrm{~A}=0.58 \mathrm{~cm} / \mathrm{s}, * \mathrm{~B}=0.22 \mathrm{~cm} / \mathrm{s}$ \\
\hline $\mathrm{D}_{\mathrm{e}}$ & $* \mathrm{~A}=0.11 \mathrm{~cm}^{2} / \mathrm{s},{ }^{*} \mathrm{~B}=0.031 \mathrm{~cm}^{2} / \mathrm{s}$ \\
\hline$\rho_{O C}$ & $5204 \mathrm{~kg} / \mathrm{m}^{3}$ \\
\hline$C_{H_{2}}$ & $.03 \mathrm{~kg} / \mathrm{m}^{3}$ \\
\hline $\mathrm{L}$ & $\mathrm{A}=209 \mathrm{~nm} \mathrm{~B}=86 \mathrm{~nm}$ \\
\hline $\mathrm{R}$ & $5 \mu \mathrm{m}$ \\
\hline $\mathrm{b}$ & $\mathrm{A}=0.74, \mathrm{~B}=0.8$ \\
\hline
\end{tabular}

The reaction rate constant and effective diffusion co-efficient were then tested with the Reynolds number, Schmidt number, Sherwood number, Thiele modulus, Weisz-Prater criteria, and effectiveness factor defined in Table 7.5 and shown in Table 7.6 to ensure the reactor conditions were free from mass transport limitations.

Table 7.5. Definition of dimensionless diffusive and chemical reaction coefficients

\begin{tabular}{|c|c|}
\hline Reaction Dimensionless Coefficient & Equation \\
\hline Reynolds Number (Re) & $\frac{d_{p} * U * \rho_{g}}{\mu}$ \\
\hline Schmidt Number (Sc) & $\frac{\mu}{\rho * D_{e}}$ \\
\hline Sherwood Number (Sh) & $\frac{k_{m} * d_{O C}}{D_{e}}$ \\
\hline Thiele Modulus ( $*$ ) & $\frac{k_{S} * \rho_{O C} * S A}{D_{e}}$ \\
\hline Weisz-Prater Criteria (WP) & $\frac{A R R * R^{2}}{D_{e} * C_{g}}$ \\
\hline Effectiveness Factor $(\eta)$ & $\frac{3}{\Phi^{2}} *(\Phi * \operatorname{coth}(\Phi)-1)$ \\
\hline
\end{tabular}


Where $d_{p}, U, \rho_{g}, \mu, D_{e}, k_{m}, d_{O C}, R_{O C}, k_{S}, \rho_{O C}, S A, A R R, R, C_{g}$ represented pipe diameter, gas velocity, reaction gas density, viscosity, effective diffusion, gas-film mass transfer co-efficient, diameter of the $\mathrm{OC}$, radius of the $\mathrm{OC}$, surface reaction constant, density of the OC, surface area of the OC, apparent reaction rate, reaction surface radius, and reaction gas concentration, respectively.

Dimensionless analysis of parameters derived from the 1-D grain boundary model determined that the reactor conditions provided parameters free from mass transport limitations. As shown in Table 7.6, the Reynolds number was calculated with the optimized reaction conditions and indicated that flow was laminar across the pellet and the reaction gas concentration could be considered constant. To evaluate the ability of the reaction gas to penetrate the pellet, the Schmidt number was also calculated. Using the effective diffusion calculated from the 1-D diffusion model with experimental TGA data at $800{ }^{\circ} \mathrm{C}$, a Schmidt number 0.04 was calculated which indicated that molecular diffusion of the reaction gas through the pellet far exceeded any concentration gradient in the hydrodynamic boundary layer. This further emphasized that with the given reaction conditions, internal and external diffusion limitations could be neglected. The mass transfer reaction rate was calculated from the Sherwood number and it was determined that the reaction was not controlled by any concentration gradients in the gas-film surrounding the pellet. 
Table 7.6. Dimensionless mass transport analysis of reactor conditions

\begin{tabular}{|c|c|}
\hline Parameter & Value \\
\hline Reynolds & 6.0 \\
\hline Schmidt & 0.04 \\
\hline Sherwood & 2.3 \\
\hline$\mu\left(\right.$ Viscosity $\left.\mathrm{H}_{2} 800{ }^{\circ} \mathrm{C}\right)$ & $0.000019 \frac{\mathrm{kg}}{\mathrm{ms}}$ \\
\hline$\rho_{\mathrm{g}}\left(\right.$ Density at $800{ }^{\circ} \mathrm{C} 40 \%$ Vol $\left.\mathrm{H}_{2}\right)$ & $0.45 \frac{\mathrm{kg}}{\mathrm{m}^{3}}$ \\
\hline U (Flow Velocity) & $0.84 \frac{\mathrm{m}}{\mathrm{s}}$ \\
\hline Pipe Diameter & $0.16 \mathrm{~cm}$ \\
\hline Average Particle Radius & $300 \mu \mathrm{mm}$ \\
\hline Mass Transfer Reaction Rate & $4.8 \frac{\mathrm{Mol} \mathrm{O}}{\mathrm{m}^{2} \mathrm{~s}}$ \\
\hline Apparent Mass Transfer Reaction Rate & $0.002 \frac{\mathrm{Mol} \mathrm{O}}{\mathrm{m}^{2} \mathrm{~s}}$ \\
\hline
\end{tabular}

As shown by the data in Table 7.7, the Weisz-Prater criteria, Thiele Modulus, and effectiveness factor were also calculated from the pre-described reactor conditions and reaction constants obtained from 1-D phase boundary model analysis to ensure that the reactions occurring in the particles were free from diffusional mass transport limitations as well. A Weisz-Prater criterion $<1$ and an effectiveness factor derived from the Thiele Modulus $>.9$ further emphasized that reaction parameters obtained from these experiments were free from any internal or external diffusional limitations, and that the reaction parameters obtained from this analysis could be considered intrinsic constants. 
Table 7.7 Dimensionless particle mass transport analysis

\begin{tabular}{|c|c|c|}
\hline Parameter & $\mathrm{CuFeO}_{2}$ & $\mathrm{Cu}_{0.5} \mathrm{Mn}_{1.5} \mathrm{FeO}_{4}$ \\
\hline Thiele & 0.51 & .73 \\
\hline Weisz-Prater & 0.39 & .24 \\
\hline Effectiveness Factor & 0.99 & .97 \\
\hline Apparent Reaction Rate & $3099 \frac{\mathrm{MolO} O}{\mathrm{~m}^{3} \mathrm{~s}}$ & $500 \frac{\mathrm{MolO}}{\mathrm{m}^{3} \mathrm{~s}}$ \\
\hline
\end{tabular}

After careful consideration the effects from internal or external mass transfer limitations, the diffusional resistance can be neglected in the calculate parameters and intrinsic rate constants are shown in

Determination of important kinetic parameters such as $\mathrm{k}_{0}$, frequency factor, and $\mathrm{E}_{\mathrm{a}}$, activation energy, were investigated using the Arrhenius Equation, as shown in Equation 4.22, for the reaction rate " $\mathrm{k}(\mathrm{T})$."

Table 7.8 from the temperatures tested.

Determination of important kinetic parameters such as $\mathrm{k}_{0}$, frequency factor, and $\mathrm{E}_{\mathrm{a}}$, activation energy, were investigated using the Arrhenius Equation, as shown in Equation 4.22, for the reaction rate " $\mathrm{k}(\mathrm{T})$."

Table 7.8. Intrinsic reaction parameters obtained from Arrhenius curves

\begin{tabular}{|c|c|c|}
\hline Temperature (K) & Reaction Rate Constant A & Reaction Rate Constant B \\
\hline 973 & $5.0 \times 10^{-4} \frac{\mathrm{m}}{\mathrm{s}}$ & $2.2 \times 10^{-4} \frac{\mathrm{m}}{\mathrm{s}}$ \\
\hline 1023 & $5.4 \times 10^{-4} \frac{\mathrm{m}}{\mathrm{s}}$ & $3.0 \times 10^{-4} \frac{\mathrm{m}}{\mathrm{s}}$ \\
\hline 1073 & $5.8 \times 10^{-4} \frac{\mathrm{m}}{\mathrm{s}}$ & $3.8 \times 10^{-4} \frac{\mathrm{m}}{\mathrm{s}}$ \\
\hline
\end{tabular}




\begin{tabular}{|c|c|c|}
\hline 1123 & $6.2 \times 10^{-4} \frac{\mathrm{m}}{\mathrm{s}}$ & $4.6 \times 10^{-4} \frac{\mathrm{m}}{\mathrm{s}}$ \\
\hline 1173 & $6.2 \times 10^{-4} \frac{\mathrm{m}}{\mathrm{s}}$ & $5.0 \times 10^{-4} \frac{\mathrm{m}}{\mathrm{s}}$ \\
\hline Activation Energy $\left(\mathrm{E}_{\mathrm{a}}\right)$ & $11.2 \frac{\mathrm{KJ}}{\mathrm{mol}}$ & $39.1 \frac{\mathrm{KJ}}{\mathrm{mol}}$ \\
\hline Frequency Factor $\left(\mathrm{k}_{0}\right)$ & $2.0 \times 10^{-3} \frac{\mathrm{m}}{\mathrm{s}}$ & $2.9 \times 10^{-2} \frac{\mathrm{m}}{\mathrm{s}}$ \\
\hline
\end{tabular}

Activation energy and frequency factors for the two phases were determined by plotting $\ln (k) v s . \frac{1}{T}$ for the desired temperature range shown in Figure 7.10. The Arrhenius constants derived from Figure 7.10 were then inserted into Equation 7.7 for reaction prediction.

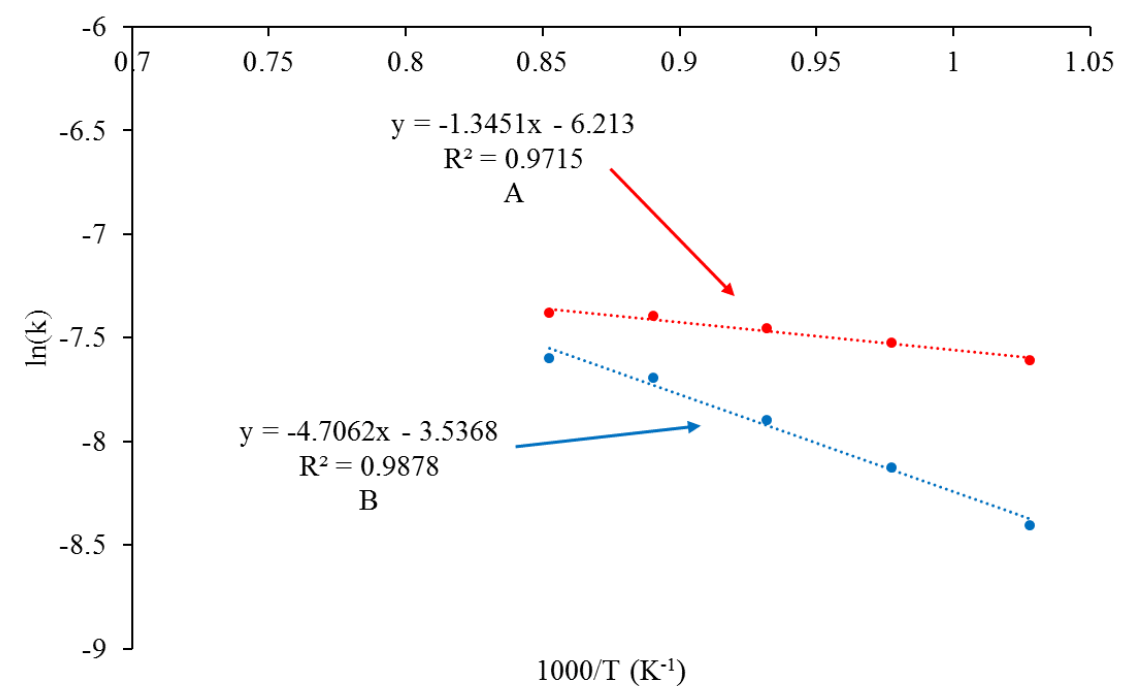

Figure 7.10. Arrhenius curves obtained from reaction modeling

As shown in Figure 7.10, and Table 7.8, intrinsic activation energies of $11.2 \mathrm{KJ} / \mathrm{mol}$ and $39.1 \mathrm{KJ} / \mathrm{mol}$ were calculated for reactions involving the two phases observed from XRD analysis. These values agreed well with the model-free analysis performed earlier. Initially $\mathrm{CuFeO}_{2}$ dominated the reaction as indicated by the closeness in value of the activation energies calculated from model-free and isothermal analyses. As this reaction progressed, the reaction properties of $\mathrm{Cu}_{0.5} \mathrm{Mn}_{1.5} \mathrm{FeO}_{4}$ eventually dominated the reduction pathway. At approximately $60 \%$ conversion, 
this phase dominated the reaction as indicated by the corresponding change in activation energy during model-free analysis.

$$
X=\varphi_{A}\left(1-e^{-\left(\frac{C_{H_{2}}}{\rho * L} * e^{-\frac{980.9}{T}}\right)^{2}}\right)+\varphi_{B}\left(1-e^{-\left(\frac{C_{H_{2}}}{\rho * L} * e^{-\frac{7447.3}{T}}\right)^{2}}\right)
$$

Reaction parameters obtained from 1-D grain boundary model analysis agree extremely well with the nucleation and growth base to provide an excellent reaction prediction as shown in Figure 7.11.

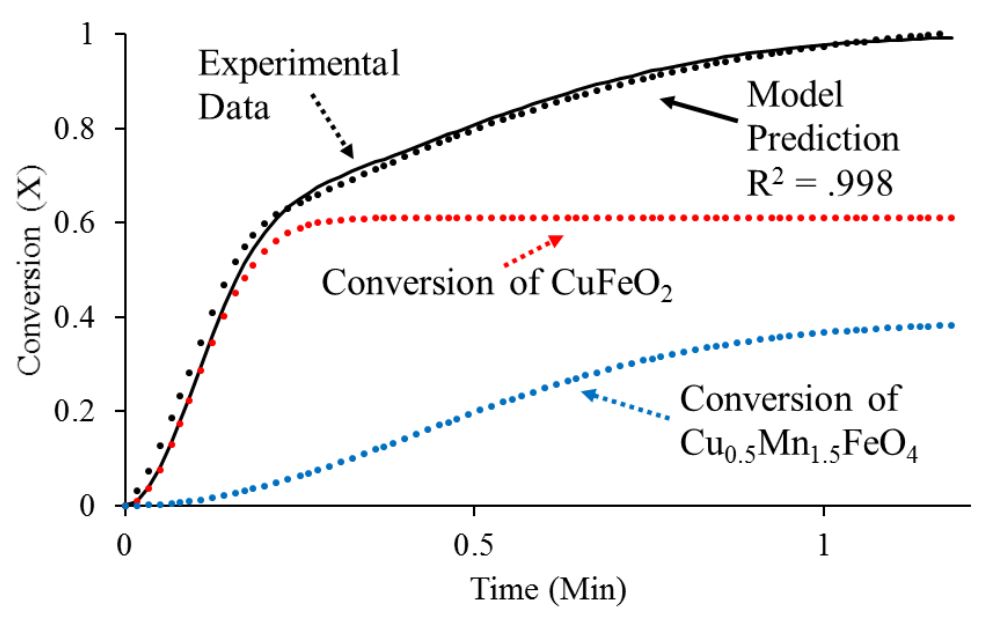

Figure 7.11. Model prediction with derived reaction parameters

As shown in Table 7.9, deconvoluted activation energies during $\mathrm{H} 2$ reduction with the trimetallic OC were calculated. Values of $11.2 \mathrm{KJ} / \mathrm{mol}$ and $39.1 \mathrm{KJ} / \mathrm{mol}$ are reported for Phase A, $\mathrm{CuFeO}_{2}$, and $\mathrm{Phase} \mathrm{B}, \mathrm{Cu}_{0.5} \mathrm{Mn}_{1.5} \mathrm{FeO}_{4}$, respectively. The values determined in this analysis are lower than that found in literature. As shown previously in Figure 7.1 and Figure 7.2, reduction reactions with $\mathrm{H}_{2}$ initiated at extremely low temperatures with observed exothermic heat flow. Given the ability of the synergistic trimetallic system to perform reduction reactions with $\mathrm{H}_{2}$ at low temperatures, the influence of temperature should not have a significant effect on this phase at elevated temperatures where CLC operations would occur. Furthermore, the Cu-containing 
material listed in Table 7.9 was not tested above $800{ }^{\circ} \mathrm{C}$ as the authors encountered a change in the reduction mechanism. The reduction mechanism of the trimetallic system was consistent throughout the temperatures tested and the additional temperatures tested in the operational regime of a CLC reactor likely improved the accuracy of the values obtained. In addition, reaction parameters calculated from this analysis were the result of unique species with enhanced observed reactivity compared to that of the single and bimetallic counterparts. Thus, low activation energies are the result of the enhanced observed reactivity of the complex trimetallic system. To validate the reported activation energies, it was vital to determine that there were no mass transport limitations from the experimental setup or pellets so that the values obtained could be considered intrinsic.

Table 7.9. Comparison of reaction parameters obtained from the current work and other values found in literature

\begin{tabular}{|c|c|c|c|c|}
\hline Reference & Oxygen Carrier & Model & $\begin{array}{c}\text { Activation } \\
\text { Energy } \\
(\mathrm{KJ} / \mathrm{mol})\end{array}$ & $\begin{array}{c}\text { Frequency } \\
\text { Factor }\end{array}$ \\
\hline Current & $\mathrm{CuFeO}_{2}$ (A) & 1-D Grain Boundary & 11.9 & $9 \times 10^{-1} \frac{\mathrm{m}}{\mathrm{s}}$ \\
\hline Current & $\mathrm{Cu}_{0.5} \mathrm{Mn}_{1.5} \mathrm{FeO}_{4}(\mathrm{~B})$ & 1-D Grain Boundary & 46.1 & $4 \times 10^{0} \frac{\mathrm{m}}{\mathrm{s}}$ \\
\hline$[82]$ & $10 \mathrm{wt} \%{\mathrm{CuO} / \mathrm{Al}_{2} \mathrm{O}_{3}}$ & 1-D Grain Boundary & 33.0 & $1 \times 10^{-4}\left(\frac{\mathrm{m}}{\mathrm{s}}\right)$ \\
\hline$[83]$ & $\mathrm{Pure} \mathrm{Fe}_{2} \mathrm{O}_{3}$ & 2-D Unreacted Core & 85.4 & - \\
\hline$[83]$ & $10 \% \mathrm{NiO} / \mathrm{Fe}_{2} \mathrm{O}_{3}$ & 2-D Unreacted Core & 56.5 & - \\
\hline$[84]$ & $\mathrm{Ilmenite}$ & 3-D Grain Boundary & 65.0 & $6.2 \times 10^{-2}\left(\frac{\mathrm{m}}{\mathrm{s}}\right)$ \\
\hline$[85]$ & $\mathrm{Fe}_{2} \mathrm{O}_{3}$ & $\begin{array}{c}\text { Nucleation and } \\
\text { Growth }\end{array}$ & 64.2 & $\mathrm{~N} . \mathrm{R}$. \\
\hline$[86]$ & $\mathrm{CaMn}_{0.9} \mathrm{Mn}_{0.1} \mathrm{O}_{3-\delta}$ & $\begin{array}{c}\text { 1-D Film and } \\
\text { Diffusion }\end{array}$ & 84.7 & $72\left(\frac{1}{\mathrm{~s}}\right)$ \\
\hline
\end{tabular}


Chapter 8. Reaction Characterization and Kinetic Modeling during the Reduction of $\mathrm{CuFeMnO}_{4}$ with $\mathrm{CO}$

\subsection{DSC/TGA during TPR with CO Performance Data Comparison of $\mathrm{CuFeMnO}_{4}$ and Corresponding Single Metal Oxides}

As shown by the heat flow, weight change, and global reaction rate data in Figure 8.1AC, respectively, DSC/TGA during TPR with 20\% CO balance Ar were conducted to compare the reduction pathway of the trimetallic OC with that of the corresponding single metal counterparts
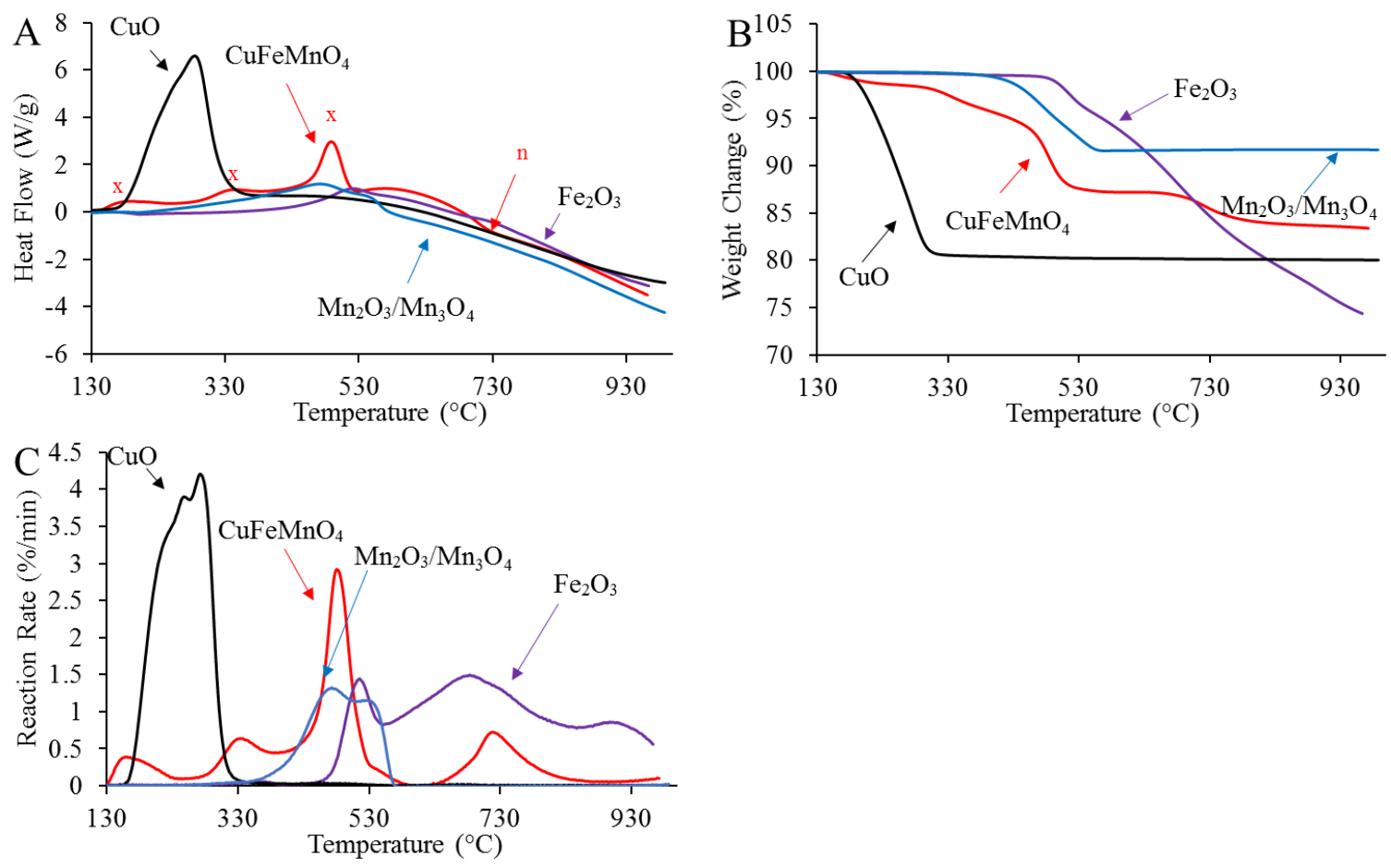

Figure 8.1. DSC/TGA during TPR with 20\% CO balance Ar performance data comparison of $\mathrm{CuFeMnO}_{4}$ and corresponding pure metal oxides

According to the DSC/TGA during TPR with 20\% CO balance Ar data shown in Figure 8.1, the reduction of $\mathrm{CuFeMnO}_{4}$ initiated at approximately $141{ }^{\circ} \mathrm{C}$ which was demonstrated by exothermic heat flow, labeled, " $x$ " for exothermic, in Figure 8.1A and a simultaneous local rate maximum shown in Figure 8.1C at the observed temperature. The reaction rate data shown in Figure 8.1C is the differentiation of the weight change profiles shown in Figure 8.1B. This reaction 
at $141{ }^{\circ} \mathrm{C}$ was followed by another slightly exothermic reaction at $332^{\circ} \mathrm{C}$, then another highly exothermic reaction at $485{ }^{\circ} \mathrm{C}$, and a final endothermic reaction was observed at $729^{\circ} \mathrm{C}$, labeled, " $n$ " for endothermic. In addition to identifying unique reaction profiles, experimentally observed exothermic heat flow during reduction also suggested that auto-thermal operation with this OC could be more easily sustained.

As shown by the DSC/TGA during TPR with CO data in Figure 8.1, the reduction pathway of $\mathrm{CuFeMnO}_{4}$ with $\mathrm{CO}$ demonstrated unique heat flow and corresponding reaction rate maxima which were different from that with $\mathrm{CuO}, \mathrm{Fe}_{2} \mathrm{O}_{3}$, and $\mathrm{Mn}_{2} \mathrm{O}_{3} / \mathrm{Mn}_{3} \mathrm{O}_{4}$. The reduction of $\mathrm{CuO}$ initiated at $254{ }^{\circ} \mathrm{C}$ followed quickly by another exothermic reaction rate maxima at $276{ }^{\circ} \mathrm{C}$. The reduction of $\mathrm{CuO}$ to $\mathrm{Cu}$ was rapid and produced a substantial amount of exothermic data, but similar reaction data was not observed with $\mathrm{CuFeMnO}_{4}$ which indicated that $\mathrm{CuO}$ was not a dominant phase in the trimetallic OC. The reduction of $\mathrm{Fe}_{2} \mathrm{O}_{3} \rightarrow \mathrm{Fe}_{3} \mathrm{O}_{4}$ with $\mathrm{CO}$ initiated at 522 ${ }^{\circ} \mathrm{C}$ with a slightly exothermic reaction. This was followed by energy-neutral reactions at $691{ }^{\circ} \mathrm{C}$ and $915{ }^{\circ} \mathrm{C}$ which were indicative of the endothermic reduction of $\mathrm{Fe}_{3} \mathrm{O}_{4} \rightarrow \mathrm{FeO}$ and exothermic reaction of $\mathrm{FeO} \rightarrow \mathrm{Fe}$. These unique reactions demonstrated that the reduced iron species was reactive at elevated temperatures, however, these reactions were different from that with $\mathrm{CuFeMnO}_{4}$. The reduction of $\mathrm{Mn}_{2} \mathrm{O}_{3} / \mathrm{Mn}_{3} \mathrm{O}_{4}$ with $\mathrm{CO}$ initiated exothermically at $468{ }^{\circ} \mathrm{C}$ and was followed by another exothermic reduction at $542{ }^{\circ} \mathrm{C}$ which was indicative of the reduction of $\mathrm{Mn}_{2} \mathrm{O}_{3} \rightarrow \mathrm{Mn}_{3} \mathrm{O}_{4}$ and $\mathrm{Mn}_{3} \mathrm{O}_{4} \rightarrow \mathrm{MnO}$. According to the calculated FactSage thermochemical data in Table 8.1, the reduction of pure $\mathrm{Mn}_{2} \mathrm{O}_{3}$ was highly exothermic, however this was not observed during DSC/TGA analysis of the single metal Mn-oxide due to the possible coexistence of $\mathrm{Mn}_{2} \mathrm{O}_{3}$ and $\mathrm{Mn}_{3} \mathrm{O}_{4}$ phases after calcination. Heat flow data and reaction rate data suggested that $\mathrm{Mn}$ in the trimetallic system may have contributed additional exothermic heat flow which was not 
observed in the single metal Mn-oxide system. According to the heat flow and reaction rate data, the presence of pure $\mathrm{Mn}_{2} \mathrm{O}_{3} / \mathrm{Mn}_{3} \mathrm{O}_{4}$ did not represent a dominant phase in $\mathrm{CuFeMnO}$.

Table 8.1. Theoretical enthalpy of reaction calculation with pure metal oxides and CO

\begin{tabular}{|c|c|}
\hline Reaction & $\Delta \mathrm{H}_{800}(\mathrm{KJ})$ \\
\hline $2 \mathrm{CuO}+\mathrm{CO} \rightarrow \mathrm{Cu}_{2} \mathrm{O}+\mathrm{CO}_{2}$ & $-150.0 \mathrm{KJ}$ \\
\hline $\mathrm{Cu}_{2} \mathrm{O}+\mathrm{CO} \rightarrow 2 \mathrm{Cu}+\mathrm{CO}_{2}$ & $-115.1 \mathrm{KJ}$ \\
\hline $3 \mathrm{Fe}_{2} \mathrm{O}_{3}+\mathrm{CO} \rightarrow 2 \mathrm{Fe}_{3} \mathrm{O}_{4}+\mathrm{CO}_{2}$ & $-39.3 \mathrm{KJ}$ \\
\hline $\mathrm{Fe}_{3} \mathrm{O}_{4}+\mathrm{CO} \rightarrow 3 \mathrm{FeO}+\mathrm{CO}_{2}$ & $+16.4 \mathrm{KJ}$ \\
\hline $\mathrm{FeO}+\mathrm{CO} \rightarrow \mathrm{Fe}+\mathrm{CO}_{2}$ & $-17.9 \mathrm{KJ}$ \\
\hline $3 \mathrm{Mn}_{2} \mathrm{O}_{3}+\mathrm{CO} \rightarrow 2 \mathrm{Mn}_{3} \mathrm{O}_{4}+\mathrm{CO}_{2}$ & $-210.2 \mathrm{KJ}$ \\
\hline $\mathrm{Mn}_{3} \mathrm{O}_{4}+\mathrm{CO} \rightarrow 3 \mathrm{MnO}+\mathrm{CO}_{2}$ & $-53.7 \mathrm{KJ}$ \\
\hline $\mathrm{MnO}+\mathrm{CO} \rightarrow \mathrm{Mn}+\mathrm{CO}_{2}$ & $103.6 \mathrm{KJ} * \Delta \mathrm{G}_{800}>0$ \\
\hline
\end{tabular}

The experimental heat flow data observed during DSC/TGA during TPR with CO qualitatively agreed with the theoretical enthalpy of reaction calculations in Table 8.1. The reduction of $\mathrm{Mn}_{2} \mathrm{O}_{3} \rightarrow \mathrm{Mn}_{3} \mathrm{O}_{4}$ with $\mathrm{CO}$ had the highest exothermic enthalpy of reaction, followed by $\mathrm{CuO} \rightarrow \mathrm{Cu}_{2} \mathrm{O}, \mathrm{Cu}_{2} \mathrm{O} \rightarrow \mathrm{Cu}, \mathrm{Mn}_{3} \mathrm{O}_{4} \rightarrow \mathrm{MnO}, \mathrm{Fe}_{2} \mathrm{O}_{3} \rightarrow \mathrm{Fe}_{3} \mathrm{O}_{4}$, and lastly $\mathrm{FeO} \rightarrow \mathrm{Fe}$. An endothermic theoretical enthalpy of reaction was calculated for the reductions of $\mathrm{Fe}_{3} \mathrm{O}_{4} \rightarrow \mathrm{FeO}$ and $\mathrm{MnO} \rightarrow \mathrm{Mn}$ however this reaction is not favorable at $800^{\circ} \mathrm{C}$. The enthalpies of reaction during reduction of $\mathrm{Fe}_{3} \mathrm{O}_{4}$ and $\mathrm{FeO}$ were approximately "equal and opposite" in value which most likely produced the energy-neutral heat flow data due to the co-existence of these phases during the TPR of $\mathrm{Fe}_{2} \mathrm{O}_{3}$. 
Table 8.2. Experimental and theoretical oxygen transfer capacities of pure metal oxides and $\mathrm{CuFeMnO}_{4}$

\begin{tabular}{|c|c|c|}
\hline Reaction & $\begin{array}{c}\text { Theoretical } \\
\text { Oxygen Transfer }\end{array}$ & $\begin{array}{c}\text { Experimental } \\
\text { Oxygen Transfer }\end{array}$ \\
\hline $\mathrm{CuO}+\mathrm{Fe}_{3} \mathrm{O}_{4}+\mathrm{Mn}_{3} \mathrm{O}_{4}+3.5 \mathrm{CO} \rightarrow \mathrm{Cu}+3 \mathrm{MnO}+3 \mathrm{Fe}+3.5 \mathrm{CO}_{2}$ & $17.9 \%$ & $16.6 \%$ \\
\hline $\mathrm{CuFeMnO}+\mathrm{CO} \rightarrow \mathrm{CuFeMnO} / 2$ CuFeMnO$+\mathrm{CO}_{2}$ & $13.4 \%-20.1 \%$ & $16.6 \%$ \\
\hline $\mathrm{CuO}+\mathrm{CO} \rightarrow \mathrm{Cu}+\mathrm{CO}_{2}$ & $20.1 \%$ & $20.0 \%$ \\
\hline $2 \mathrm{Fe}_{2} \mathrm{O}_{3}+\mathrm{CO} \rightarrow \mathrm{Fe}+2 \mathrm{CO}_{2}$ & $30.1 \%$ & $25.6 \%$ \\
\hline $2 \mathrm{Mn}_{2} \mathrm{O}_{3}+2 \mathrm{CO} \rightarrow 4 \mathrm{MnO}+\mathrm{CO}_{2}$ & $10.1 \%$ & $8.3 \%$ \\
\hline
\end{tabular}

As shown by the data in Table 8.2, the highest oxygen transfer capacity was achieved by $\mathrm{Fe}_{2} \mathrm{O}_{3}$, but the reaction initiation temperature was higher than that of the other oxides as shown in Figure 8.1. Despite the large oxygen transfer capacity, reduction of $\mathrm{Fe}_{2} \mathrm{O}_{3}$ to $\mathrm{Fe}$ was mostly endothermic and displayed slow reaction rates as shown in Figure 8.1C. The reduction of trimetallic $\mathrm{CuFeMnO}_{4}$ initiated at the lowest temperature and demonstrated improved heat flow and reaction rate data than that with $\mathrm{Fe}_{2} \mathrm{O}_{3}$ or $\mathrm{Mn}_{2} \mathrm{O}_{3}$. Although $\mathrm{CuO}$ displayed high oxygen transfer capacity and significant exothermic heat flow, agglomeration and attrition issues plague this metal oxide [35]. Incorporation of $\mathrm{Fe}_{2} \mathrm{O}_{3}$ and $\mathrm{Mn}_{2} \mathrm{O}_{3}$ with $\mathrm{CuO}$ maintained high oxygen transfer capacity exothermic heat flow, and reaction rate data during reduction. 
8.2 DSC/TGA during TPR with CO Performance Data Comparison of $\mathrm{CuFeMnO}_{4}$ and Corresponding Bimetallic Oxides

As shown by the heat flow, weight change, and reaction rate data in Figure 8.2A-C, respectively, DSC/TGA during TPR with 20\% CO balance Ar were conducted to compare the reduction pathway of the trimetallic OC with that of the corresponding bimetallic counterparts.
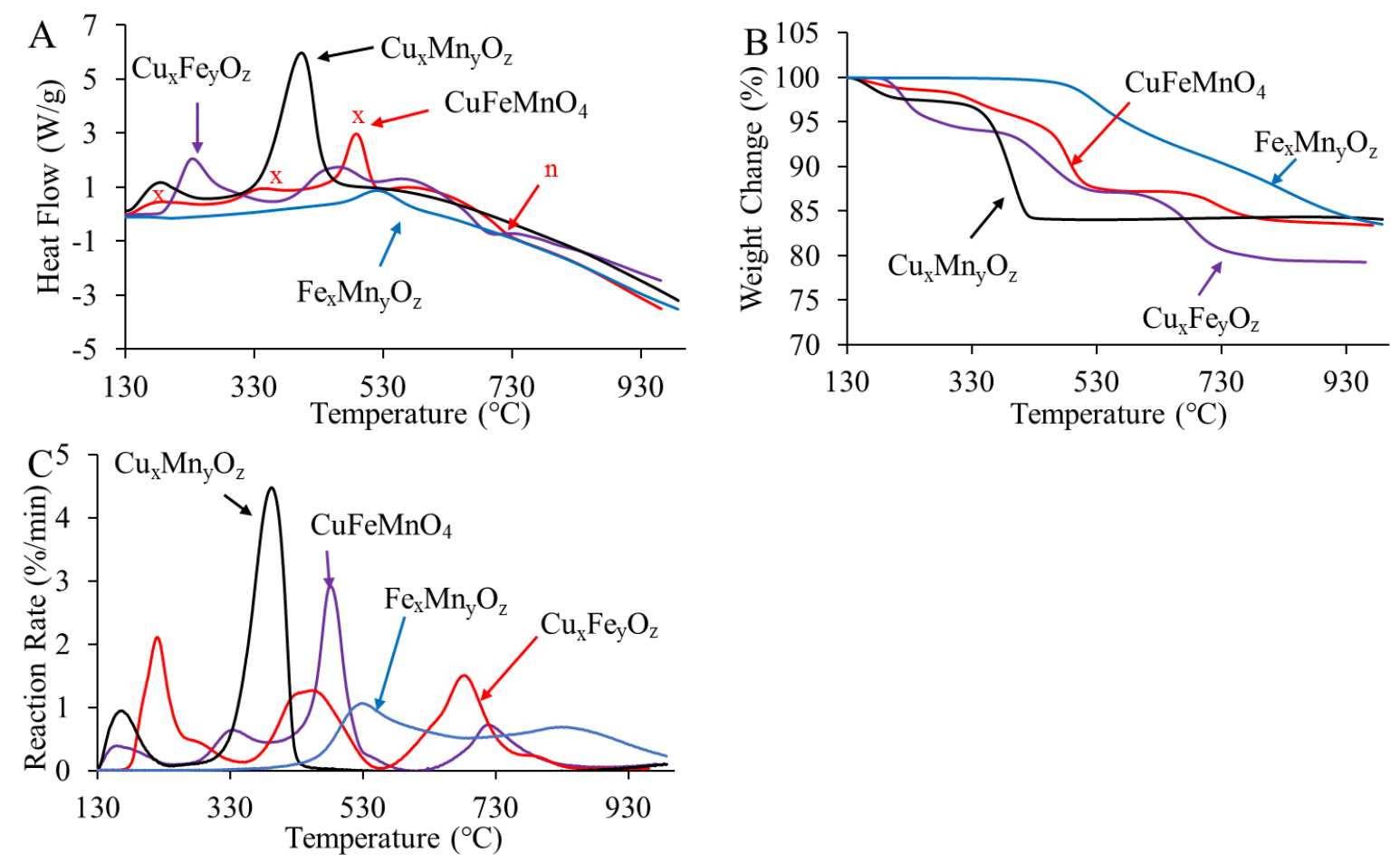

Figure 8.2. DSC/TGA during with 20\% CO balance Ar performance data comparison of $\mathrm{CuFeMnO}_{4}$ and corresponding bimetallic oxides

As shown by the data in Figure 8.2, the reduction of $\mathrm{CuFeMnO}_{4}$ initiated at the lowest temperature compared to that of the bimetallic counterparts and followed closely by bimetallic copper manganese oxide, bimetallic copper iron oxide, and the reduction of bimetallic iron manganese oxide initiated last. The initial reduction of bimetallic copper manganese oxide with $\mathrm{CO}$ at $171{ }^{\circ} \mathrm{C}$ was exothermic and this was followed by another exothermic reaction at $397{ }^{\circ} \mathrm{C}$. It appeared that incorporation of $\mathrm{Cu}$ in $\mathrm{Mn}$-based oxides promoted a highly exothermic reaction. However, the heat flow and reaction rate data differed from that with trimetallic OC which 
indicated that copper manganese oxide did not represent a dominant phase in the trimetallic OC. The reduction of copper iron oxide initiated at $224{ }^{\circ} \mathrm{C}$ with an exothermic reaction and was followed by another exothermic reduction at $461{ }^{\circ} \mathrm{C}$. Following these exothermic reactions of copper iron oxide, an endothermic reaction was observed at $670{ }^{\circ} \mathrm{C}$. The differences in heat flow data and reaction data indicated that the presence of bimetallic copper iron oxide did not represent a dominant phase in the trimetallic OC. Bimetallic iron manganese oxide initiated reduction at 544 ${ }^{\circ} \mathrm{C}$, higher than that of the other bimetallic oxides and the reaction was exothermic. This was followed by an endothermic reaction at $838^{\circ} \mathrm{C}$. The differences in heat flow data and reaction data indicated that the presence of bimetallic iron manganese did not represent a dominant phase in the trimetallic system.

Table 8.3. Experimental and theoretical oxygen transfer capacities of bimetallic oxides and $\mathrm{CuFeMnO}_{4}$

\begin{tabular}{|c|c|c|}
\hline Reaction & $\begin{array}{c}\text { Theoretical } \\
\text { Oxygen Transfer }\end{array}$ & $\begin{array}{c}\text { Experimental } \\
\text { Oxygen Transfer }\end{array}$ \\
\hline $\mathrm{CuO}+\mathrm{Fe}_{3} \mathrm{O}_{4}+\mathrm{Mn}_{3} \mathrm{O}_{4}+3.5 \mathrm{CO} \rightarrow \mathrm{Cu}+3 \mathrm{MnO}+3 \mathrm{Fe}+3.5 \mathrm{CO}_{2}$ & $17.9 \%$ & $16.6 \%$ \\
\hline $\mathrm{CuFeMnO}+\mathrm{CO} \rightarrow \mathrm{CuFeMnO} / \mathrm{CuFeMnO}+\mathrm{CO}_{2}$ & $13.4 \%-20.1 \%$ & $16.6 \%$ \\
\hline $\mathrm{CuO}+\mathrm{Fe}_{2} \mathrm{O}_{3}+2 \mathrm{CO} \rightarrow \mathrm{Cu}+\mathrm{FeO} / \mathrm{Fe}+2 \mathrm{CO}_{2}$ & $15.1 \%-25.1 \%$ & $20.7 \%$ \\
\hline $\mathrm{CuO}+\mathrm{Mn}_{2} \mathrm{O}_{3}+2 \mathrm{CO} \rightarrow \mathrm{Cu}+2 \mathrm{MnO}+2 \mathrm{CO}_{2}$ & $15.1 \%$ & $15.8 \%$ \\
\hline $\mathrm{Fe}_{2} \mathrm{O}_{3}+\mathrm{Mn}_{2} \mathrm{O}_{3}+2 \mathrm{CO} \rightarrow \mathrm{FeO} / \mathrm{Fe}+2 \mathrm{MnO}+2 \mathrm{CO}_{2}$ & $10.1 \%-20.0 \%$ & $16.5 \%$ \\
\hline
\end{tabular}

According to the data shown in Table 8.3, all bimetallic oxides and $\mathrm{CuFeMnO}_{4}$ yielded very high oxygen transfer capacities for reactions involving CO. Compared to our previous studies, the reactions with $\mathrm{CO}$ initiated at temperatures significantly lower than reactions with $\mathrm{CH}_{4}$, however, total oxygen transfer capacity during reactions with $\mathrm{CO}$ was slightly lower [73]. 


\subsection{In-situ XTD TPR with 5\% CO Balance Ar Analysis}

To further evaluate the heat flow and reaction data provided during DSC/TGA TPR with CO, in-situ XRD during TPR with CO analysis was conducted to corelate structural and chemical changes that could have occurred to the oxide.

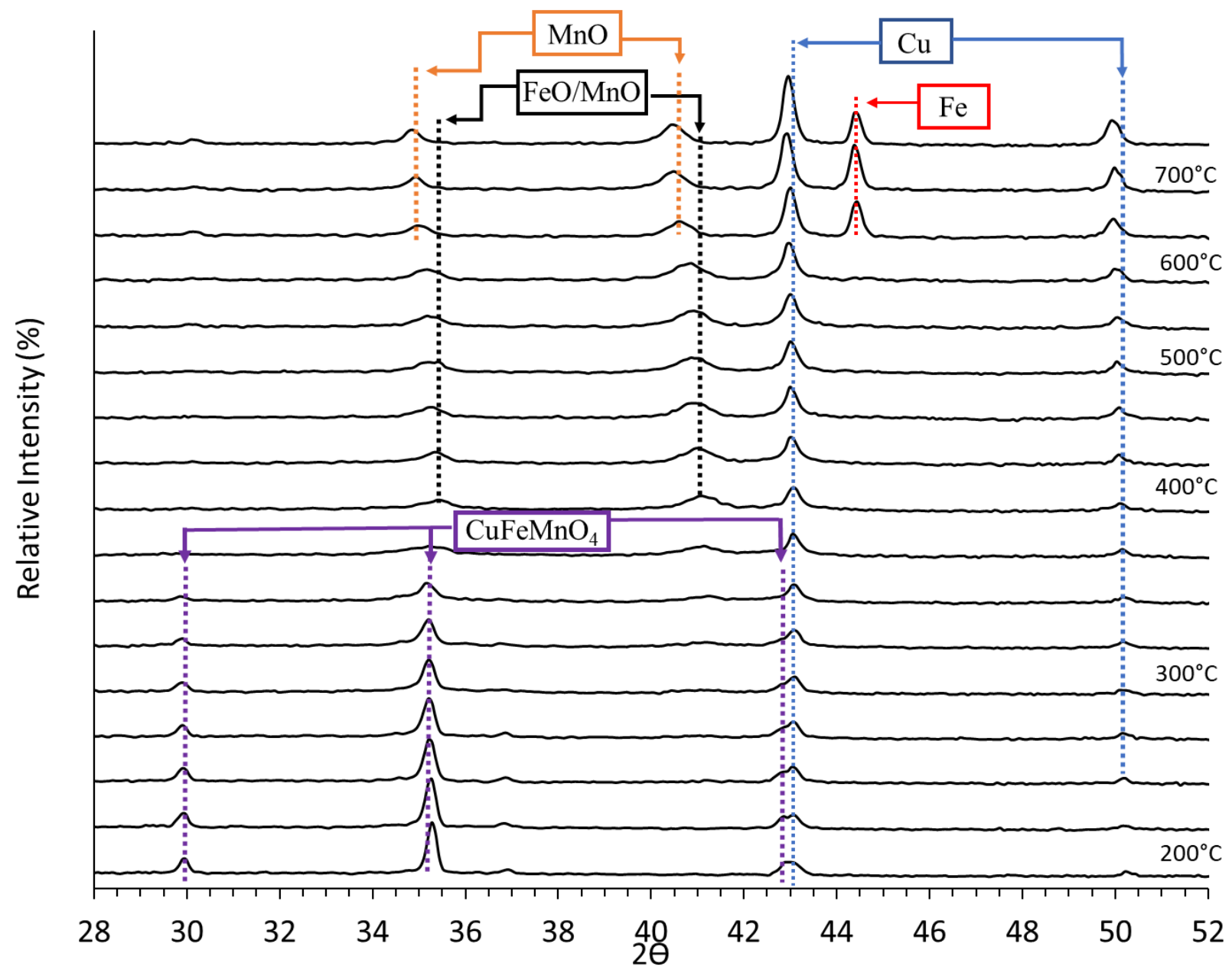

Figure 8.3. In-situ $\mathrm{XRD}_{\text {of }} \mathrm{CuFeMnO}_{4}$ during TPR with $5 \% \mathrm{CO}$

According to the data in Figure 8.3, the trimetallic phase was initially detected due to the observation of the characteristic peak of $\mathrm{CuFeMnO}_{4}$ at $35.4952 \Theta\left(\begin{array}{lll}3 & 1 & 1\end{array}\right)$ at temperatures below $200{ }^{\circ} \mathrm{C}$ while in the presence of $5 \% \mathrm{CO}$ balance Ar environment. When the reaction initiated between $\mathrm{CuFeMnO}_{4}$ and $\mathrm{CO}$ at approximately $200{ }^{\circ} \mathrm{C}$, the characteristic peak of $\mathrm{CuFeMnO}_{4}$ shifted left and significantly broadened indicating lattice expansion most likely due to the creation 
of oxygen vacancies. Simultaneously, the formation of metallic $\mathrm{Cu}$ was detected. At approximately $400{ }^{\circ} \mathrm{C}$, the formation of mixed $\mathrm{FeO}-\mathrm{MnO}$ phase was observed which indicated that oxygen bound in trimetallic phase was consumed during reactions with $\mathrm{CO}$ and a new phase with new reaction properties had formed. At approximately $650{ }^{\circ} \mathrm{C}$, metallic $\mathrm{Fe}$ and $\mathrm{MnO}$ were observed simultaneously as the peaks of the $\mathrm{FeO}-\mathrm{MnO}$ phase correspondingly disappeared. The apparent consumption of the $\mathrm{FeO}-\mathrm{MnO}$ phase to form $\mathrm{MnO}$ and $\mathrm{Fe}$ was most likely due creation of oxygen vacancies from reactions occurring with oxygen bound to $\mathrm{Fe}$ and $\mathrm{CO}$. It appeared that $\mathrm{CO}$ preferentially reacts with the cations distributed to the lattice of the trimetallic phase. $\mathrm{CO}$ reacted with oxygen from $\mathrm{Cu}^{2+}$ in the lattice of $\mathrm{CuFeMnO}_{4}$ initially, followed by a reaction with oxygen from $\mathrm{Mn}^{3+}$ and $\mathrm{Fe}^{3+}$ which contributed to the formation of the mixed $\mathrm{FeO}-\mathrm{MnO}$ phase. These initial reactions could also be associated with the exothermic reactions that were observed during DSC/TGA analysis. Thus, the endothermic reaction could be attributed to the formation of metallic Fe from the mixed $\mathrm{FeO}-\mathrm{MnO}$ phase at deeper states of reduction. Since the formation of this secondary new phase has different reaction properties, the initial reaction of the $\mathrm{CuFeMnO}_{4}$ phase with $\mathrm{CO}$ must be considered independently during reaction modeling. Additionally, this reaction was exothermic, as shown by the DSC/TGA data, which is an important feature to make the autothermal operation of a CLC reactor more feasible. 


\subsection{Isothermal in-situ XRD with Controlled CO Exposures at $800{ }^{\circ} \mathrm{C}$}

As shown by the data in Figure 8.4, isothermal in-situ XRD experiments with controlled $\mathrm{CO}$ exposures at $800{ }^{\circ} \mathrm{C}$ were conducted by incrementally dosing the sample with $5 \mathrm{~mL} \mathrm{CO}$ injections and obtaining an XRD diffractogram after each injection. Isothermal conditions were chosen to represent the ideal operation of CLC in the reducing reactor.

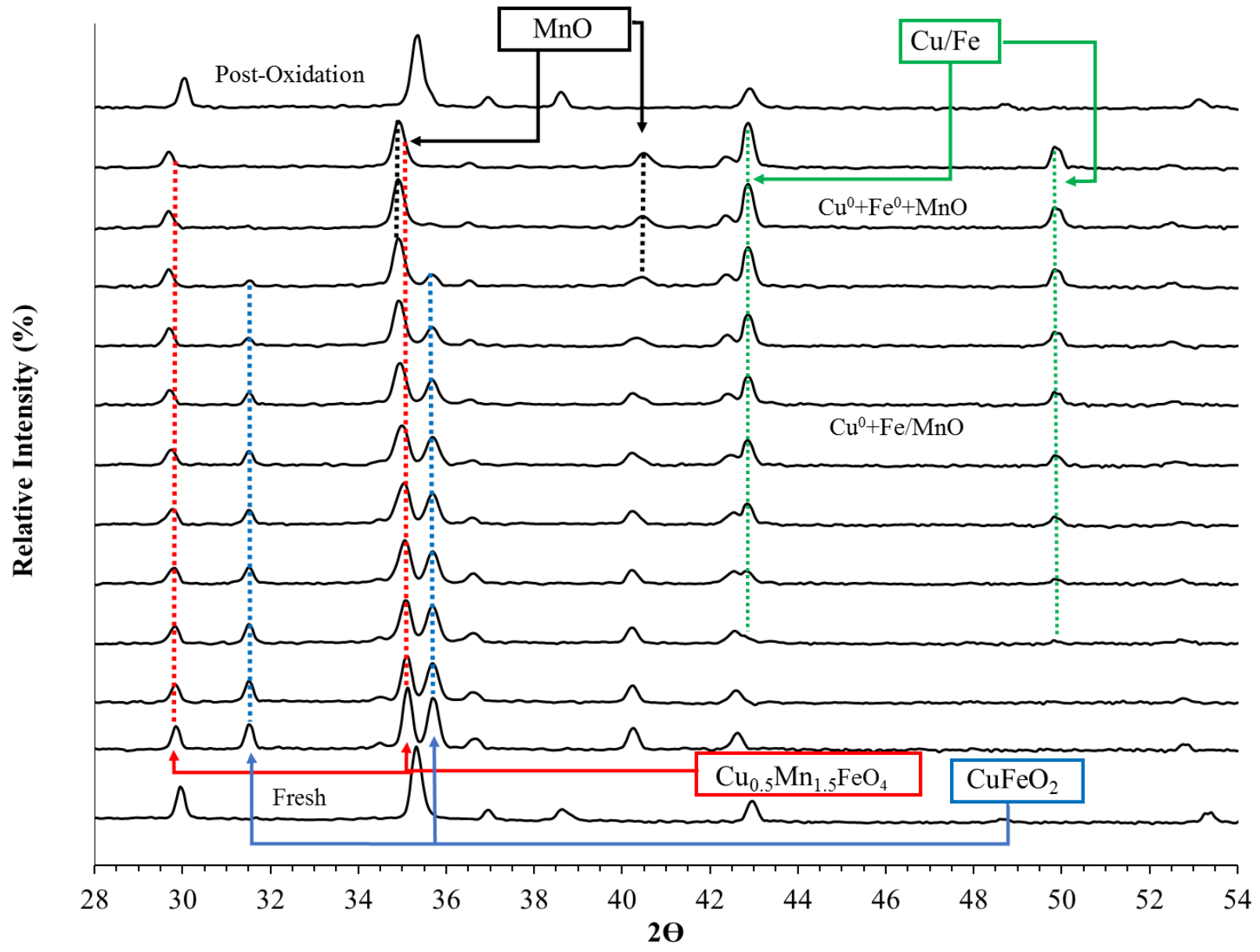

Figure 8.4. Isothermal in-situ XRD during $\mathrm{CO}$ doses at $800{ }^{\circ} \mathrm{C}$

As shown in Figure 8.4, a bimetallic copper iron-rich phase and a manganese-rich

trimetallic phase were detected after oxygen uncoupling in an inert environment at $800{ }^{\circ} \mathrm{C}$, prior to $\mathrm{CO}$ introduction. According to XRD analysis, these two phases most closely matched to a copper-iron rich phase, $\mathrm{CuFeO}_{2}$, noted as Phase $\mathrm{A}$, and a manganese-rich trimetallic phase 
$\mathrm{Cu}_{0.5} \mathrm{Mn}_{1.5} \mathrm{FeO}_{4}$ noted as Phase $\mathrm{B}$. It appeared that the initial $\mathrm{CO}$ exposures preferentially depleted oxygen from that of the copper-iron rich phase and oxygen from that phase was rapidly consumed compared to that from the phase composed of $\mathrm{Cu}_{0.5} \mathrm{Mn}_{1.5} \mathrm{FeO}_{4}$. It was apparent from isothermal in-situ XRD analysis with $\mathrm{CO}$ exposures at $800{ }^{\circ} \mathrm{C}$ shown in Figure 8.4 that two independent phases had formed after oxygen uncoupling and each possessed unique reaction properties with $\mathrm{CO}$. The copper-iron rich phase was able to reduce rapidly to metallic copper and iron and a secondary reaction with the manganese-rich trimetallic phase was also observed.

During reduction, peak broadening was more pronounced than any observed peak shifting from exposure to $\mathrm{CO}$ at $800{ }^{\circ} \mathrm{C}$. The significant amount of peak broadening suggested that the mechanism of oxygen exhaustion was controlled by the shrinkage of the crystallites rather than the volume of the bulk pellet. 


\subsection{XPS Analysis}

XPS analysis was conducted as shown in Figure 8.5 to assess the oxidative states of the cations in the fully oxidized trimetallic lattice.
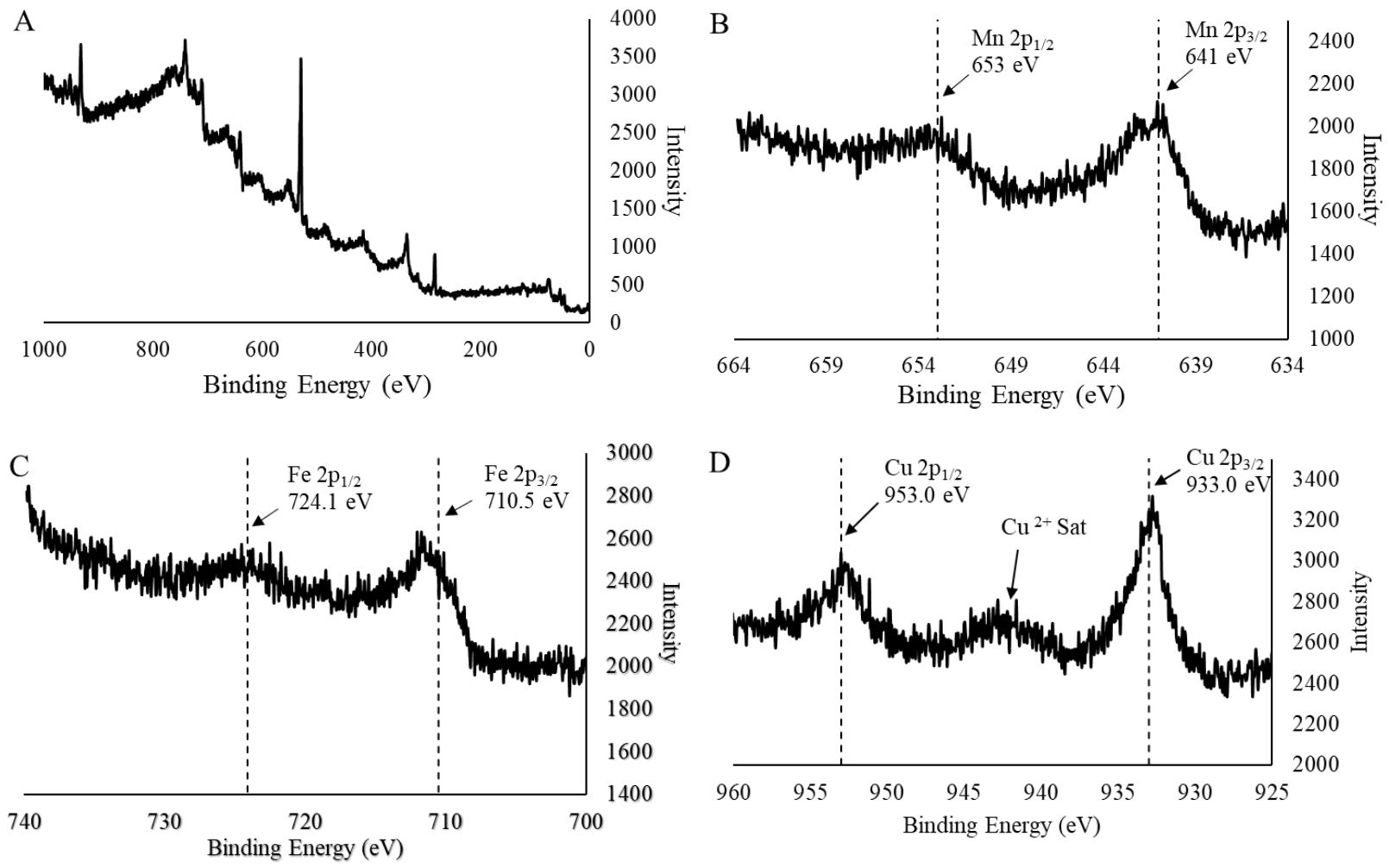

Figure 8.5. XPS analysis of $\mathrm{CuFeMnO}_{4}$ (A) Survey scan (B) Mn 2p (C) Fe 2p (D) Cu 2p

The XPS peaks corresponding to $\mathrm{Cu}, \mathrm{Mn}$, and $\mathrm{Fe}$ are shown in Figure 8.5. The binding energies of $641 \mathrm{eV}$ for $\mathrm{Mn} 2 \mathrm{p}_{3 / 2}$ and $653 \mathrm{eV}$ for $2 \mathrm{p}_{1 / 2}$ corresponded to the formation of $\mathrm{Mn}^{3+}$ [87]. The binding energies of $710.5 \mathrm{eV}$ for $\mathrm{Fe} 2 \mathrm{p}_{3 / 2}$ and $724 \mathrm{eV}$ for $2 \mathrm{p}_{1 / 2}$ corresponded to the formation of $\mathrm{Fe}^{3+}$ [88]. The binding energies of $933.0 \mathrm{eV}$ for $\mathrm{Cu} 2 \mathrm{p}_{3 / 2}$ and $953 \mathrm{eV}$ for $2 \mathrm{p}_{1 / 2}$, and the satellite peak from $940 \mathrm{eV}-945 \mathrm{eV}$ corresponded to the formation of $\mathrm{Cu}^{2+}[89]$.

Thus, according to the XPS data provided in Figure 8.5, the formation of $\mathrm{CuFeMnO}_{4}$ stabilized $\mathrm{Cu}^{2+}, \mathrm{Mn}^{3+}$, and $\mathrm{Fe}^{3+}$ in the trimetallic phase. As suggested by in-situ XRD and DSC/TGA during TPR with CO, the initial exothermic reaction can be attributed to the reduction of $\mathrm{Cu}^{2+}$. However, it is possible that the trimetallic phase promoted the stabilization of $\mathrm{Mn}^{3+}$ which 
could have provided the additional exothermic heat flow data during reduction to $\mathrm{Mn}^{2+}$ which was not observed with pure $\mathrm{Mn}_{2} \mathrm{O}_{3} / \mathrm{Mn}_{3} \mathrm{O}_{4}$. The presence of $\mathrm{Fe}^{3+}$ according to XPS data and reduction to $\mathrm{Fe}^{2+}$ could have contributed additional exothermic heat flow. It is most likely that the deeper reduction of $\mathrm{Fe}^{2+} \rightarrow \mathrm{Fe}^{0}$, according to in-situ XRD and DSC/TGA data, contributed the endothermic heat flow. 


\subsection{Spent SEM-EDS Elemental Mapping}

SEM imaging and elemental mapping was conducted to observe features on the surface and cross-sectionally of the pellet that would help provide additional information in determining a reaction model and validating the regeneration of the trimetallic phase.
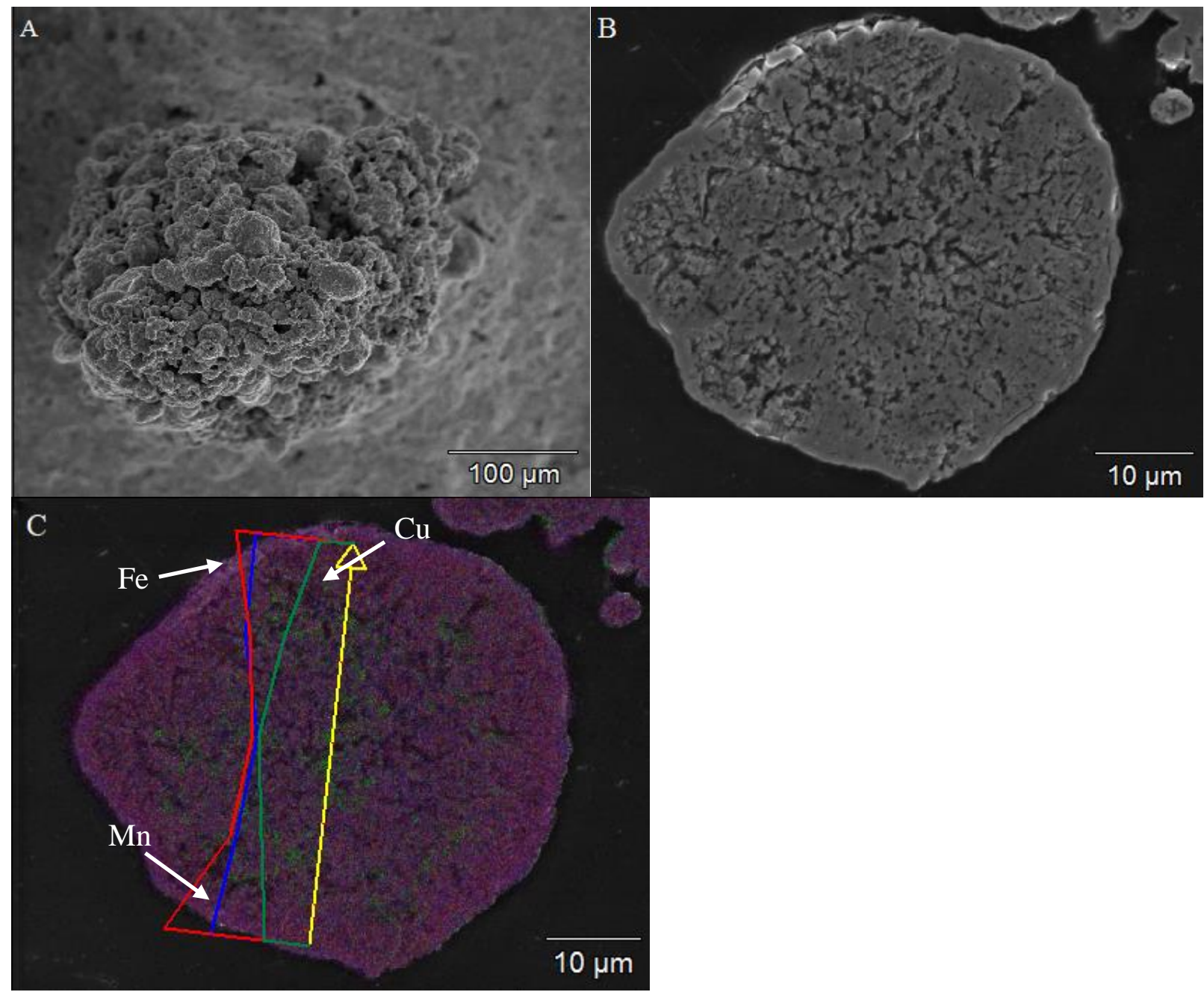

Figure 8.6. Reacted $\mathrm{CuFeMnO}_{4}$ SEM-EDS of $\mathrm{Cu}, \mathrm{Fe}$, and $\mathrm{Mn}$ are labeled green, red, and blue respectively (A) SEM exterior imaging (B) SEM cross-section imaging (C) EDS elemental mapping and line scan

SEM-EDS analysis of the reacted $\mathrm{CuFeMnO}_{4}$ pellet after the third oxidation in air of a typical CO/air cyclic redox experiment at $800{ }^{\circ} \mathrm{C}$ is shown in Figure 8.6. According to the data in Figure 8.6, the pellet underwent both chemical and physical changes during cyclic reduction and 
oxidation experiments. As shown in Figure 8.6A, the surface contour of the pellet still exhibited macro-porous features similar to that of the fresh pellet. However, in Figure 8.6B, the interior of the reacted pellets revealed more voids and channels compared to the relatively dense nature of the fresh material. Furthermore, according to the EDS elemental mapping data in Figure 8.6C, some $\mathrm{Cu}$ clusters could be found in the bulk of the material which was consistent with the detection of $\mathrm{CuO}$ from the XRD data shown previously. Although sporadic $\mathrm{Cu}$ clusters were formed in the spent pellet after cyclic reduction and oxidation testing, the bulk of the material retained the unique trimetallic phase. As shown in Figure 8.6C, the EDS line scan determined that the trimetallic phase was consistent in locations where the $\mathrm{Cu}$ clusters were not observable. Overall, the micro-features of the pellet that influenced reactivity remained intact and overall reactivity may increase due to pore formation observed in SEM cross-sectional data shown in Figure 8.6C. 


\subsection{Isothermal Reaction Model Analysis of $\mathrm{CuFeMnO}_{4}$ and $\mathrm{CO}$}

Before reaction parameters could be calculated from experimental TGA conversion-time data, reactor conditions were optimized so that rate-limiting diffusional effects from internal and externa mass transport limitations could be neglected from modeling analysis.
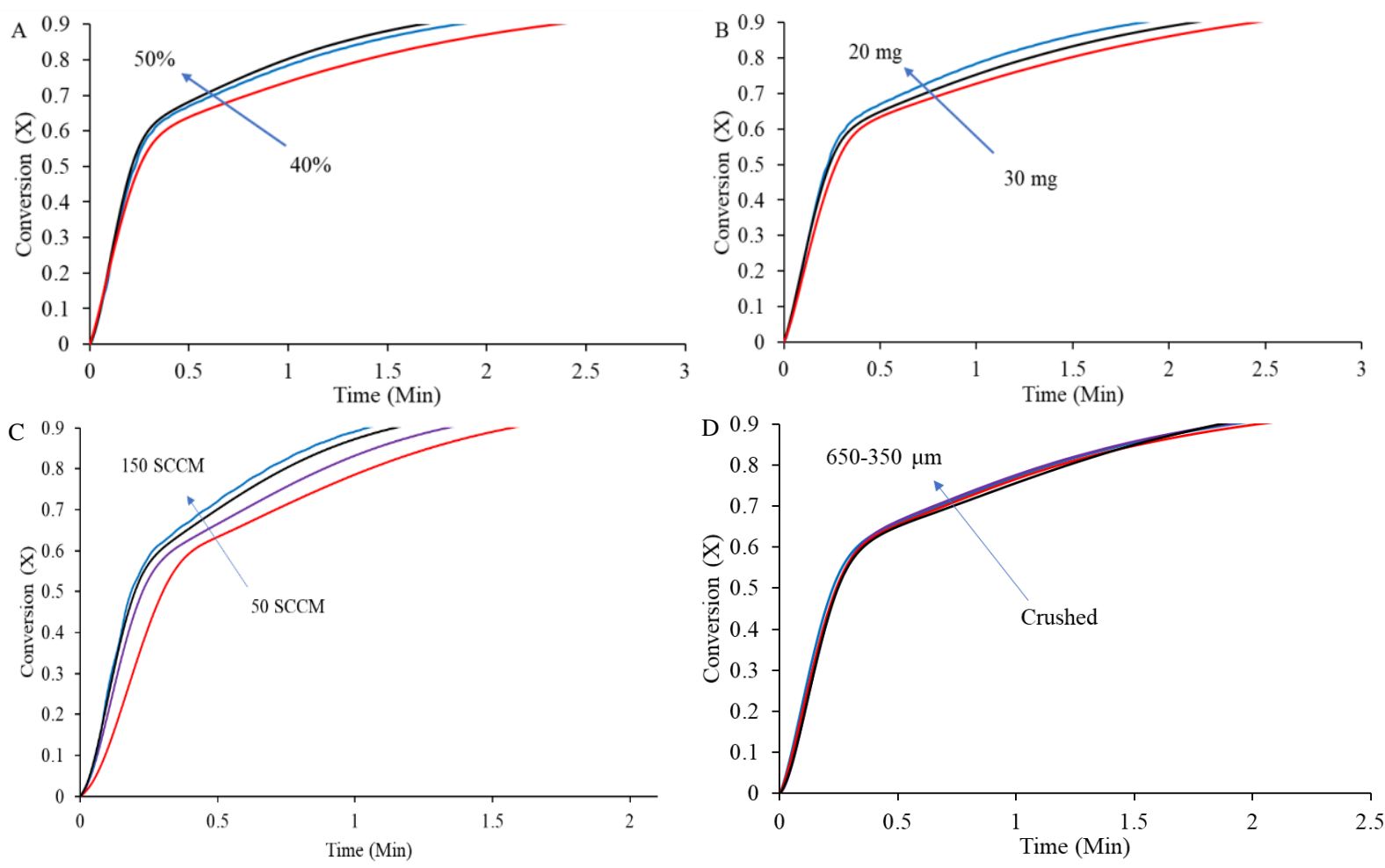

Figure 8.7. Reaction optimization during isothermal TGA CO reductions at $800{ }^{\circ} \mathrm{C}$ with respect to varying (A) Concentration (B) Mass (C) Flow rate (D) Particle size

According to the data in Figure 8.7A, $20 \mathrm{mg}$ of the trimetallic oxygen carrier was exposed to various $\mathrm{CO}$ volumetric concentrations at $125 \mathrm{SCCM}$ and 45 vol\% $\mathrm{CO}$ balance $\mathrm{Ar}$ was determined to be the optimal concentration for $\mathrm{CO}$ reaction analysis. Exposing the pellets to concentrations below this value demonstrated slower observed conversion rates and concentrations above this value had no influence on the observed conversion rate. As shown by the data in Figure 8.7B, sample mass was optimized by exposing various sample masses to 45 vol\% CO balance Ar at $125 \mathrm{SCCM}$. The reactivity of the trimetallic oxygen carrier was unaffected by charging the TGA 
reactor with sample masses below $20 \mathrm{mg}$. As demonstrated by the data in Figure 8.7C, flow rate was optimized by exposing $20 \mathrm{mg}$ of $\mathrm{CuFeMnO}_{4}$ with 45 vol\% $\mathrm{CO}$ balance Ar at various flow rates. Above 125 SCCM, external diffusional limitations in the boundary layer could be neglected. According to the data in Figure 8.7D, the effect of particle size was evaluated by exposing $20 \mathrm{mg}$ of $\mathrm{CuFeMnO}_{4}$ to $45 \mathrm{vol} \% \mathrm{CO}$ balance Ar at $100 \mathrm{SCCM}$ in ranges of $600-350 \mu \mathrm{m}, 250-350 \mu \mathrm{m}$, 180-250 $\mu \mathrm{m}$, and crushed $<74 \mu \mathrm{m}$. There was no observed change in reactivity when particle size was varied which indicated that a micro-feature of the pellet such as granule, or the grain crystallite size, was most likely the controlling shape factor during reduction reactions with $\mathrm{CO}$ as opposed to the size of the bulk pellet. This will have a significant impact on the shape factor during reaction modeling which will be addressed in the following section. 

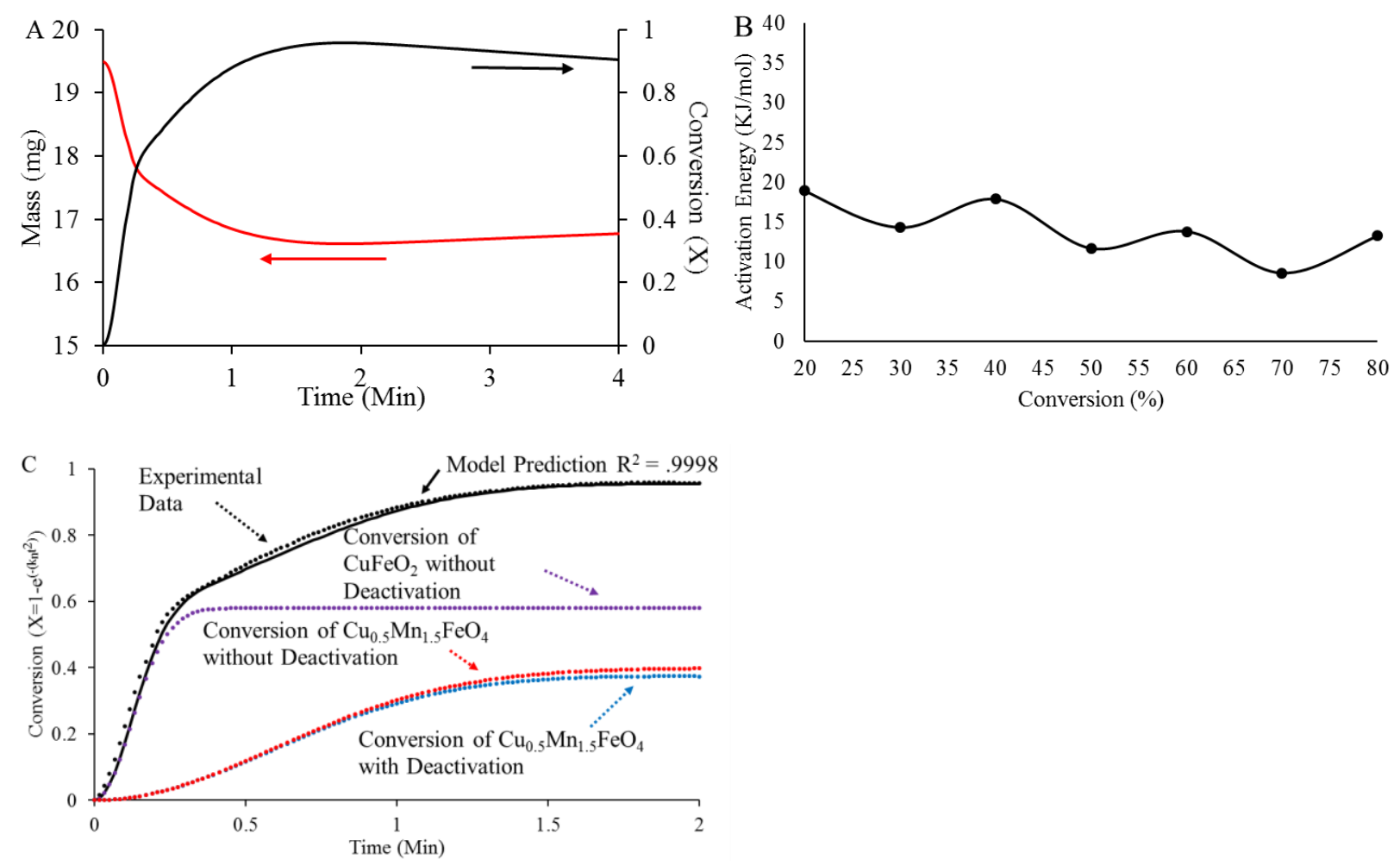

Figure 8.8. (A) TGA reduction of $20 \mathrm{mg} \mathrm{CuFeMnO} 4$ with $45 \% \mathrm{CO}$ balance $\mathrm{Ar}$ at $800^{\circ} \mathrm{C}$ (B) Model-free fitting (C) Deconvolution of phases

As shown by isothermal experimental data in Figure 8.8A, a rapid reaction occurred at $800^{\circ} \mathrm{C}$ initially with 45 vol\% $\mathrm{CO}$ as evidenced by the observed substantial weight change. Following this reaction, the observed reactivity appeared to decrease as demonstrated by the slower observed rates sustained during this portion of the reaction. Conversion was calculated using the experimental weight loss data from Figure 8.8A in Equation 4.1.

To gain a deeper understanding of the reduction pathway of $\mathrm{CuFeMnO}_{4}$ with respect to $\mathrm{CO}$, model-free analysis with conversion data collected at $900{ }^{\circ} \mathrm{C}, 850{ }^{\circ} \mathrm{C}, 800{ }^{\circ} \mathrm{C}$, and $750{ }^{\circ} \mathrm{C}$ was conducted as shown in Figure 8.8B. According to model-free analysis, the observed activation energy remained low around $\sim 15 \mathrm{KJ} / \mathrm{mol}$ throughout the duration of the reduction. According to the differences in observed reactivity from TGA analysis, it was apparent that the 
two observed phases from in-situ XRD analysis, shown in the previous section, have unique reaction properties which significantly influenced the reaction profile of $\mathrm{CuFeMnO}_{4}$ with $\mathrm{CO}$. In order to deconvolute the two phases in this reaction a parallel approach of nucleation and growth models with carbon deposition occurring during the latter stages of reduction was used to represent the two phases independently with the summation of the converted oxygen from the individual phases to yield the total oxygen depleted from the oxide as shown in Equation 8.1.

$$
X=\varphi_{A}\left(1-e^{-k_{A_{n}} t^{n_{A}}}\right)+\varphi_{B}\left(1-e^{-k_{B_{n}} t^{n_{B}}}\right) *\left(e^{k_{d_{B}} * t}\right)
$$

In Equation 8.1, X, $\varphi_{i}, \mathrm{k}_{\mathrm{n}}, \mathrm{n}, k_{C_{A}}$, and $k_{d_{B}}$ represented, conversion, oxygen density, nucleation growth rate constant, the nucleation constant, and the deactivation constant for Phase $\mathrm{A}, \mathrm{CuFeO}_{2}$, and Phase $\mathrm{B}, \mathrm{Cu}_{0.5} \mathrm{Mn}_{1.5} \mathrm{FeO}_{4}$, respectively. In order to account for carbon deposition from the Boudouard reaction, as shown by Equation 8.2, a deactivation scheme was incorporated into the reaction model. To represent the simultaneous reactions occurring at the surface of the oxygen carrier once carbon deposition occurred during the later stages of reduction, a first order reaction with respect to the reaction gas and the exposed metallic species of the reduced carrier was assumed as shown in Equation 8.3.

$$
\begin{gathered}
2 C O \rightleftarrows C+C O_{2} \Delta \mathrm{H}^{800}=-16.6 \mathrm{KJ} \\
X_{B}=\varphi_{B}\left(1-e^{-k_{B} t^{n_{B}}}\right)-\varphi_{B}\left(1-e^{-k_{B} t^{n_{B}}}\right)\left(1-e^{k_{C_{B}} t}\right)
\end{gathered}
$$

Where $X_{B}$ represented the conversion balance with respect to $\mathrm{Cu}_{0.5} \mathrm{Mn}_{1.5} \mathrm{FeO}_{4}$. After solving for like terms, Equation 8.3 simplifies to Equation 8.4 and Equation 8.5 where carbon deposition can be accounted for with respect due to $1^{\text {st }}$ order deactivation during reaction. 


$$
\begin{gathered}
X_{B}=\varphi_{B}\left(1-e^{-k_{B_{n}} t^{n_{B}}}\right)\left(1-\left(1-e^{k_{C_{B}} t}\right)\right. \\
X_{B}=\varphi_{B}\left(1-e^{-k_{B_{n}} t^{n_{B}}}\right)\left(e^{k_{C_{B}} t}\right)
\end{gathered}
$$

For reaction modeling, oxygen density was predetermined from Rietveld analysis and the theoretical oxygen transfer capacities of the two phases with respect to the total observed oxygen transfer capacity. For $\mathrm{CuFeO}_{2}$, oxygen density was fixed to $60 \%$ and $\mathrm{Cu}_{0.5} \mathrm{Mn}_{1.5} \mathrm{FeO}_{4}$ at $40 \%$. The nucleation constant was also held constant at 2, which is indicative of "plate-like" geometry, as shown in Figure 8.8 [61]. With the phases deconvoluted, 1-D grain boundary reaction control and 1-D diffusional control models, were utilized to calculate reaction and effective diffusion coefficients. These models represented the reaction front as nucleation and growth within the crystallites was occurring. This type of system has been described previously as the grainy pellet model where the reactions that occur on the surface of the grain are representative of conversion throughout the bulk of the pellet [57]. These models also provide established reaction constants with established values which can be used for Arrhenius plots, and tested with dimensionless mass transport coefficients to determine the intrinsic nature of the calculated values. These 1-D parameters were then re-inserted into the frame work of nucleation and growth reaction models to provide the necessary functionality required for the sigmoidal reduction conversion curve. Surface reaction constants and effective diffusion coefficients of $\mathrm{CuFeO}_{2}$ during reduction with $\mathrm{CO}$ were obtained from the slopes of Equation 7.3 and Equation 7.4 displayed in Figure 8.9 [80]. These calculated constants were then tested with established mass transport dimensionless parameters to confirm the intrinsic nature of the values obtained from model analysis shown previously in Table $7.5[81]$ 

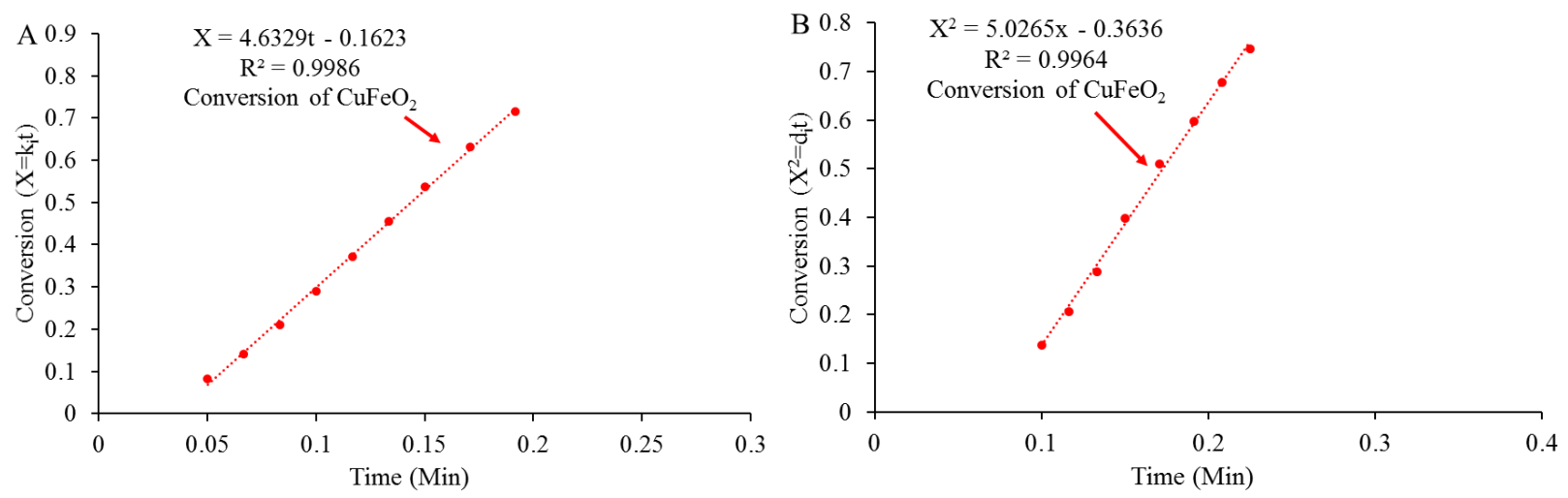

Figure 8.9. Determination of rate constants from (A) 1-D grain boundary (B) 1-D diffusional reaction models during TGA $45 \% \mathrm{CO}$ reduction at $800^{\circ} \mathrm{C}$

As shown in Figure 8.9, Phase $\mathrm{A}$ or $\mathrm{CuFeO}_{2}$, exhibited excellent linear fit with the 1-D models. The reaction rate constant, effective diffusion co-efficient, and reaction optimization conditions shown in Table 8.4 were then tested with the Reynolds number, Schmidt number, Sherwood number, to ensure reactor conditions did not produce any mass transport limitations. Thiele modulus, Weisz-Prater criteria, and effectiveness factor were also evaluated to ensure there were no mass transport limitations within the pellet. 
Table 8.4. Reaction Parameters Obtained from Model Analysis and reactor conditions from TGA experimental data at $800{ }^{\circ} \mathrm{C}$ and $125 \mathrm{SCCM}$

\begin{tabular}{|c|c|}
\hline Parameter & Value (*Measured from Figure 8.9) \\
\hline$k_{s}$ & $0.01 \mathrm{~cm} / \mathrm{s}^{*}$ \\
\hline$D_{e}$ & $0.02 \mathrm{~cm}^{2} / \mathrm{s}^{*}$ \\
\hline$\rho$ & $5204 \mathrm{~kg} / \mathrm{m}^{3}$ \\
\hline$C_{C O}$ & $.14 \mathrm{~kg} / \mathrm{m}^{3}$ \\
\hline$L$ & $209 \mathrm{~nm}$ \\
\hline$R$ & $5 \mu \mathrm{m}$ \\
\hline$b$ & .76 \\
\hline
\end{tabular}

According to dimensionless mass transport analysis performed in Table 8.5 on the experimental reaction data shown in Table 8.4, there no mass transport limitations due to the pellet or the reactor conditions. 
Table 8.5. Dimensionless mass transport analysis of reaction parameters and reactor conditions

\begin{tabular}{|c|c|}
\hline Parameter & Value \\
\hline Reynolds & 3.0 \\
\hline Schmidt & 0.45 \\
\hline Sherwood & 2.9 \\
\hline Thiele & 0.49 \\
\hline Weisz-Prater & 0.98 \\
\hline Effectiveness Factor & 0.98 \\
\hline$\mu\left(\right.$ Viscosity $\left.\mathrm{CO} 800^{\circ} \mathrm{C}\right)$ & $0.000042 \frac{\mathrm{kg}}{\mathrm{ms}}$ \\
\hline$\rho_{\mathrm{g}}\left(\right.$ Density at $\left.800^{\circ} \mathrm{C} 45 \% \mathrm{Vol} \mathrm{CO}\right)$ & $0.40 \frac{\mathrm{kg}}{\mathrm{m}^{3}}$ \\
\hline U (Flow Velocity) & $1.06 \frac{\mathrm{m}}{\mathrm{s}}$ \\
\hline Pipe Diameter & $0.16 \mathrm{~cm}$ \\
\hline Average Particle Diameter & $300 \mu \mathrm{m}$ \\
\hline Apparent Reaction Rate & $1855 \frac{\mathrm{Mol} \mathrm{O}}{\mathrm{m}^{3} \mathrm{~s}}$ \\
\hline Mass Transfer Reaction Rate & $1.2 \frac{\mathrm{MolO}}{\mathrm{m}^{2} \mathrm{~s}}$ \\
\hline Apparent Mass Transfer Reaction Rate & $0.001 \frac{\mathrm{MolO} O}{\mathrm{~m}^{2} \mathrm{~s}}$ \\
\hline
\end{tabular}

As shown in Table 8.5, the Reynolds number was calculated with the optimized reaction conditions and indicated that flow was laminar across the pellet and that the reaction gas concentration could be considered constant. To evaluate the ability of the reaction gas to penetrate the pellet, the Schmidt number was also calculated. Using the effective diffusion calculated from the 1-D diffusion model with experimental TGA data at $800{ }^{\circ} \mathrm{C}$, a Schmidt number 0.46 was 
calculated which indicated that molecular diffusion of the reaction gas through the pellet far exceeded any concentration gradient in the hydrodynamic boundary layer. This further emphasized that with the given reaction conditions, internal and external diffusion limitations could be neglected. The mass transfer reaction rate was calculated from the Sherwood number and determined that the reaction was not controlled by any concentration gradients in the gas-film surrounding the pellet. The Weisz-Prater criteria, Thiele Modulus, and effectiveness factor were also calculated from the reaction parameters obtain from reactor optimization and model analysis. A Weisz-Prater criterion $<1$ and an effectiveness factor derived from the Thiele Modulus $>.9$ further emphasized that reaction parameters obtained from these experiments were free from any internal or external diffusional limitations, and that the reaction parameters obtained from this analysis could be consider intrinsic constants. 
Table 8.6. Intrinsic reaction parameters obtained from reaction modeling and Arrhenius curves

\begin{tabular}{|c|c|c|c|}
\hline Temperature (K) & $\begin{array}{c}\text { Reaction Rate } \\
\text { Constant A }\end{array}$ & $\begin{array}{c}\text { Reaction Rate } \\
\text { Constant B }\end{array}$ & Deactivation Constant \\
\hline 1023 & $9.7 \times 10^{-5} \frac{\mathrm{m}}{\mathrm{s}}$ & $6.2 \times 10^{-5} \frac{\mathrm{m}}{\mathrm{s}}$ & $0.12 \frac{1}{s}$ \\
\hline 1073 & $1.1 \times 10^{-4} \frac{\mathrm{m}}{\mathrm{s}}$ & $6.9 \times 10^{-5} \frac{\mathrm{m}}{\mathrm{s}}$ & $0.38 \frac{1}{s}$ \\
\hline 1123 & $1.2 \times 10^{-4} \frac{\mathrm{m}}{\mathrm{s}}$ & $7.6 \times 10^{-5} \frac{\mathrm{m}}{\mathrm{s}}$ & $.008352 \frac{1}{s}$ \\
\hline 1173 & $1.3 \times 10^{-4} \frac{\mathrm{m}}{\mathrm{s}}$ & $8.0 \times 10^{-5} \frac{\mathrm{m}}{\mathrm{s}}$ & 0 \\
\hline Activation Energy $\left(\mathrm{E}_{\mathrm{a}}\right)$ & $16.2 \frac{\mathrm{KJ}}{\mathrm{mol}}$ & $16.6 \frac{\mathrm{KJ}}{\mathrm{mol}}$ & $-252.7 \frac{\mathrm{KJ}}{\mathrm{mol}}$ \\
\hline Frequency Factor $\left(\mathrm{k}_{0}\right)$ & $7.4 \times 10^{-4} \frac{\mathrm{m}}{\mathrm{s}}$ & $2.8 \times 10^{-4} \frac{\mathrm{m}}{\mathrm{s}}$ & $1.6 \times 10^{-14} \frac{1}{s}$ \\
\hline
\end{tabular}

Surface reaction constants obtained from reaction modeling are shown in Table 8.6 and these values were used to produce the Arrhenius curves for the conversion of A and B shown in Figure 8.10. The reaction deactivation constant demonstrated first order kinetics with respect to the Boudouard reaction which was depositing carbon on the surface of the reduce carrier at later stages in reduction. This reaction is not favorable at elevated temperatures and was not observed during reduction at $900{ }^{\circ} \mathrm{C}$. 


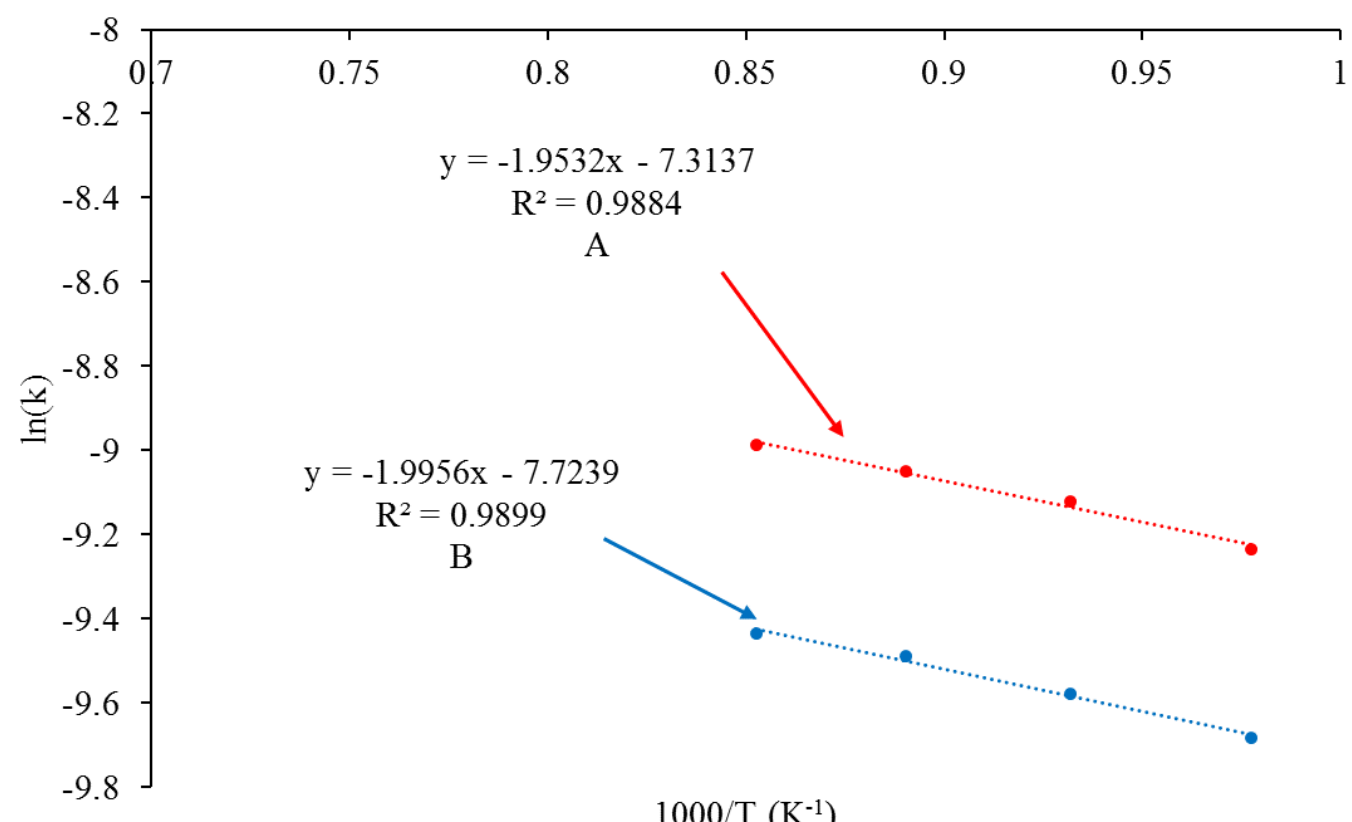

Figure 8.10. Arrhenius curves from rate constants obtained from reaction modeling According to the Arrhenius curves shown in Figure 8.10, activation energies of approximately $16.5 \mathrm{KJ} / \mathrm{mol}$ were determined from model analysis. These activation energies agreed well with model-free analysis which demonstrated that the activation energy remained relatively constant around $\sim 16 \mathrm{KJ} / \mathrm{mol}$ throughout the duration of the reduction of the trimetallic carrier with CO. It is possible mass transport limitations could have existed at later stages of reduction due product layer diffusion from decomposed carbon, however, it is unlikely that an OC would be exposed to such deep stages of reduction during CLC operation.

$$
\begin{gathered}
X=\varphi_{A}\left(1-e^{-\left(\frac{C_{C O}}{\rho * L} * 0.00074 * e^{\left.\left(\frac{1953}{T}\right) * t\right)^{2}}\right)}+\varphi_{B}\left(1-e^{-\left(\frac{C_{C O}}{\rho * L} * 0.00028 * e^{\left(\frac{1995}{T}\right)} t\right)^{2}}\right) *\right. \\
\left(e^{-0.00044 * e^{\frac{1995}{T} * t}}\right)
\end{gathered}
$$

According to the model prediction from Equation 8.6 and shown in Figure 8.11, nucleation growth modeled at the boundary of the crystallites that form the metal oxide exhibited 
an excellent correlation with experimental TGA reaction data at $800{ }^{\circ} \mathrm{C}$ during reduction with 45 vol\% CO. Limitations caused by internal and external diffusion during reduction of $\mathrm{CuFeO}_{2}$ within the trimetallic system can be neglected in current form of this model and intrinsic rate constants for the portion of reduction have been provided for CLC particle-scale reaction modeling. Carbon deposition from the Boudouard reaction correlated well with first order deactivation and its inhibitory effect during later stages of reduction has been incorporated.

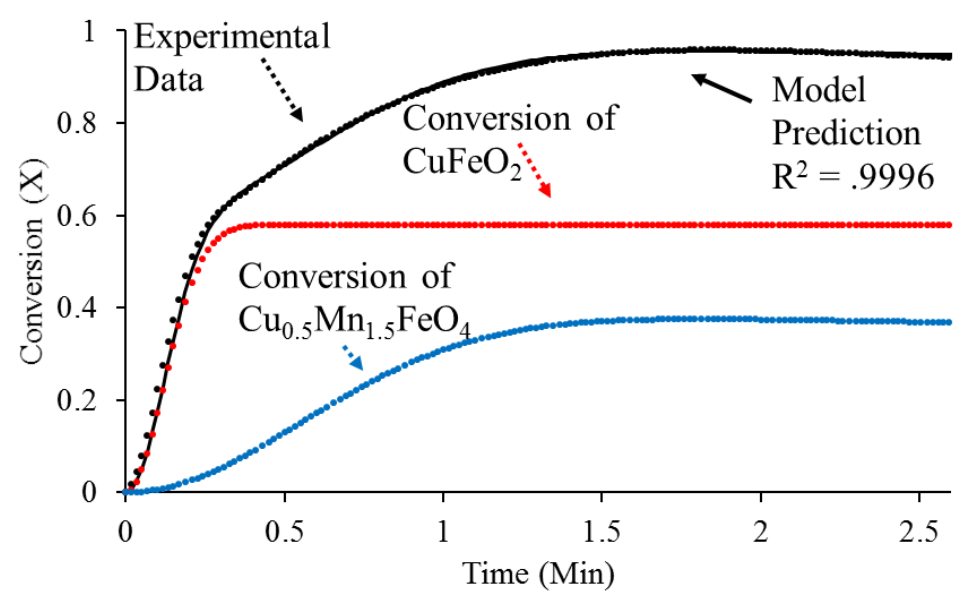

Figure 8.11. Nucleation and growth grain boundary model prediction 
Table 8.7 Comparison of activation energies

\begin{tabular}{|c|c|c|c|}
\hline Reference & Metal Oxide & Model Description & $\begin{array}{c}\text { Activation Energy } \\
(\mathrm{KJ} / \mathrm{mol})\end{array}$ \\
\hline Current & $\mathrm{CuFeO}_{2}$ (A) & $\begin{array}{c}\text { Nucleation and Growth 1-D } \\
\text { Phase Boundary }\end{array}$ & 16.2 \\
\hline Current & $\mathrm{Cu}_{0.5} \mathrm{Mn}_{1.5} \mathrm{FeO}_{4}(\mathrm{~B})$ & $\begin{array}{c}\text { Nucleation and Growth 1-D } \\
\text { Phase Boundary }\end{array}$ & 16.6 \\
\hline$[86]$ & $\mathrm{CaMn}_{0.9 \mathrm{Mg}_{0.1} \mathrm{O}_{3-\delta}}$ & Shrinking Core & $39.7-44.9$ \\
\hline$[79]$ & $\mathrm{Fe}_{2} \mathrm{O}_{3}$ & Phase Boundary & 19.0 \\
\hline$[90]$ & $\mathrm{Fe}_{2} \mathrm{O}_{3}$ & Chemical Reaction Controls & 19.8 \\
\hline
\end{tabular}

According to the data in Table 8.7, the activation energies calculated with 1-D phase boundary within the framework of nucleation growth kinetics during reduction of $\mathrm{CuFeMnO}_{4}$ with $\mathrm{CO}$ provided activation energies that agreed with values found for $\mathrm{Fe}_{2} \mathrm{O}_{3}$. According to the TPR data shown previously in Figure 8.1 and Figure 8.2, the trimetallic oxygen carrier initiated reduction at temperatures below $300{ }^{\circ} \mathrm{C}$. For a metal oxide to initiate reduction at such low temperatures, it is logical that temperature dependency would decrease as reaction temperatures increase above $750^{\circ} \mathrm{C}$ where CLC operation would occur. Upon further scrutiny, the intrinsic nature of the reaction parameters during reduction of $\mathrm{CuFeO}_{2}$ were tested and it was determined that these values were free from any mass transport limitations. 


\section{Chapter 9. Conclusions and Future Recommendations}

The purpose of this research was to develop a high performing oxygen carrier and construct reaction models for process design and scale-up. This practical guide to oxygen carrier development determined that a unique trimetallic oxygen carrier was capable of withstanding the extreme physical and chemical forces that an oxygen carrier could exhibit during CLC reactions. Upon exceeding the four requirements of a suitable oxygen carrier during the development stage, it became apparent that the trimetallic system was an ideal candidate for CLC and reaction models for large-scale simulation and process design were necessary given the promising performance of the oxygen carrier.

Trimetallic $\mathrm{CuFeMnO}_{4}$ formed a unique phase after calcination of metal oxide precursors as demonstrated by a distinct reduction pathway determined from characteristic heat flow and reaction data during DSC/TGA TPR with $\mathrm{CH}_{4}$, that differed from that with the corresponding single or bimetallic components. During reduction with $\mathrm{CH}_{4}$, the initial low temperature exothermic reduction was due to the presence of $\mathrm{CuFeMnO}_{4} \cdot \mathrm{CuFeMnO}_{4}$ initiated reduction at the lowest temperature among the various oxygen carriers studied which indicated that the trimetallic system possessed the highest reactivity. The initial reduction was also exothermic which indicated that this oxygen carrier could sustain auto-thermal operation by providing the necessary heat in the fuel reactor to sustain a continuous CLC operation. This is incredibly advantageous for reactor design as the heat produced during air oxidation can go to electricity generation and minimal heat must be transferred back to the reducing reactor. As reduction of the trimetallic oxygen carrier progressed during the TPR with $\mathrm{CH}_{4}$, a solid-solid phase transition formed an oxygen depleted phase that contributed additional endothermic data during the reduction with $\mathrm{CH}_{4}$. The ability of the trimetallic oxygen carrier to form the second intermediate phase allowed $\mathrm{CH}_{4}$ to access 
additional reactive oxygen and achieve deeper levels of reduction. These deeper reductions of $\mathrm{Cu}$, Fe and Mn oxides were due to synergistic effects of the trimetallic system.

Once it could be concluded that the high performing features observed during CLC operation with this oxygen carrier were due to the formation of the trimetallic system, reaction modeling was performed to provide reaction parameters for process scale up and to gain a deeper understanding the mechanisms that are contributing to the enhanced reactivity. The nucleation and growth with 1-D grain boundary model most accurately described the reduction mechanism the promising trimetallic OC. DSC/TGA data indicated that the trimetallic phase reduced exothermically at very low temperatures with $\mathrm{H}_{2}$ most likely due to the presence of $\mathrm{Cu}^{2+}$ which could advantageously promote auto-thermal operation in CLC reactor operating with this OC. Formation of this highly reactive significantly influenced reactivity. In-situ XRD analysis indicated the formation of $\mathrm{CuFeO}_{2}$ and $\mathrm{Cu}_{0.5} \mathrm{Mn}_{1.5} \mathrm{FeO}_{4}$ after an inert purge and that the reduction mechanism can be attributed to 1-D nucleation and growth within the crystallites as depicted in Figure 9.1. Reaction analysis of the experimental data provided valuable intrinsic rate constants which can be used for reactor modeling and scale-up. 


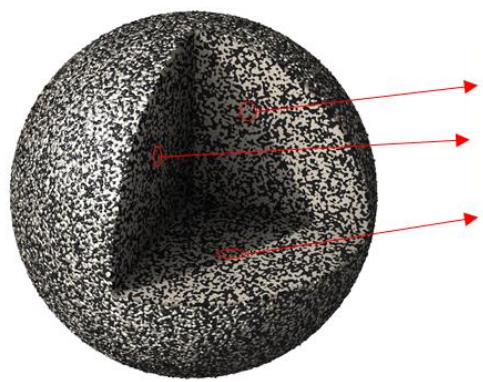

Fresh $\mathrm{CuFeO}_{2}$ and $\mathrm{Cu}_{0.5} \mathrm{Mn}_{1.5} \mathrm{FeO}_{4}$

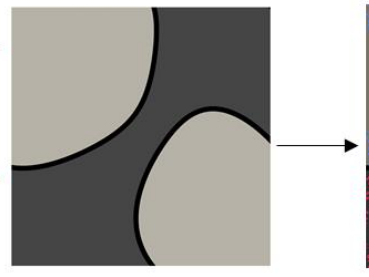

1. Fresh Unreacted Pellet

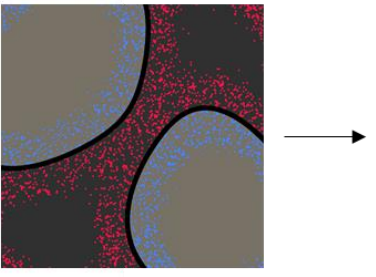

2. Induction of Germ Nuclei

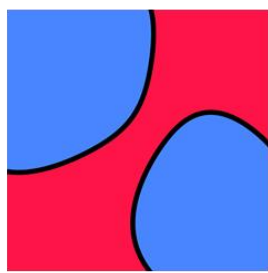

5. Converted Pellet

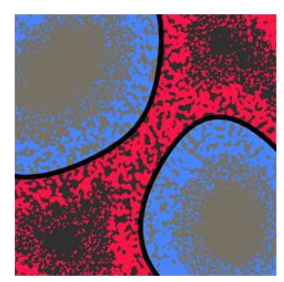

3. Growth of Nuclei

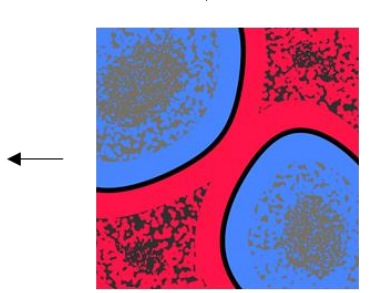

4. Coalescence of Nuclei

Figure 9.1. Particle-scale description of 1-D phase boundary nucleation and growth kinetics

The JMAEK nucleation and growth with 1-D phase boundary model accurately described the reduction mechanism of $\mathrm{CuFeMnO}_{4}$, although the consideration of carbon deposition from the Boudouard reaction required a modification to account for the inhibitive behavior of this reaction. In Figure 9.1, $\mathrm{CuFeO}_{2}$ is represented by the conversion of black to red, and $\mathrm{Cu}_{0.5} \mathrm{Mn}_{1.5} \mathrm{FeO}_{4}$ is represented by the conversion of gray to blue. DSC/TGA and XPS data indicated that the trimetallic phase reduced exothermically with $\mathrm{CO}$ due to the presence of $\mathrm{Cu}^{2+}, \mathrm{Fe}^{3+}$, and $\mathrm{Mn}^{3+}$ which is an advantage in CLC reactor operation with this OC to maintain auto-thermal operation. In-situ XRD analysis indicated that the reduction of $\mathrm{CuFeMnO}_{4}$ can be attributed to the depletion oxygen from crystallite within the metal oxide. Valuable intrinsic reaction parameters were obtained from reaction analysis and a model for large-scale design and simulation was developed.

It is the opinion of this study that the trimetallic system is a suitable carrier for CLC and could bridge the final gap to large scale operation of this technology. To date, the ideal candidate 
for large scale operation has yet to be determine, but according to the results from this work, the trimetallic oxygen carrier $\mathrm{CuFeMnO}_{4}$ has the potential to be this candidate.

\subsection{Future Recommendations}

The analysis conducted in this work identified a promising candidate for CLC of fossil fuels. However, there is more to be learned from this carrier and additional large-scale design criteria to be considered. (1) The thermal transport properties throughout the carrier during reduction and oxidation reactions must be calculated in order to better estimate circulation rate in a large-scale system. The amount of heat that can be transferred between reactors must be known prior to successful large-scale tests. Satisfying the heat balance in the dual reactor system will have major impact in successful large-scale demonstrations where auto-thermal operation is achieved. (2) Although the reduction kinetics are typically considered the most important part in the design concept of large scale reactors, oxidation kinetics would also provide valuable information in reactor sizing. (3) The fate of S/HCL and other contaminations from coal gasification or impurities in the feed stream must be studied to better predict reaction deactivation kinetics, material longevity, and carry over between reactors from adsorbed or chemisorbed impurities. 


\section{Chapter 10. References}

[1] S. Soloman, G. K. Plattner, R. Knutti and Friedlingstein, "Irreversible Climate Change due to Carbon Dioxide Emissions," Proceedings of the National Academy of Sciences of the United States of America, vol. 106, pp. 1704-1709, 2009.

[2] F. BIrol, "CO2 Emissions from Fuel Combustion," International Energy Agency, p. 10, 2017.

[3] P. Nejat, F. Jomehzadeh, M. Taheri, M. Gohari and M. Majid, "A Global Review of Energy Consumption, CO2 Emissions and Policy in the Residential Sector (with an Overview of the Top Ten CO2 Emitting Countries)," Renewable and Sustainable Energy Reviews, vol. 43, pp. 843-862, 2014.

[4] "U.S. Energy Information Administration," 10 May 2017. [Online]. Available: https://www.eia.gov/energyexplained/index.cfm?page=electricity_in_the_united_states.

[5] E. Rubin, H. Mantripragada, A. Marks, P. Versteeg and J. Kitchin, "The Outlook for Improved Carbon Capture Technology," Progress in Energy and Combustion Science, vol. 38, pp. 630-671, 2012.

[6] B. Dutcher, M. Fan and A. Russel, "Amine-Based CO2 Capture Technology Development from the Begining of 2013- A Review," Applied Materials \& Interfaces, vol. 7, pp. 2137$2148,2015$.

[7] Z. Zhou, Z. You, Z. Wang, X. Hu, J. Zhou and K. Cen, "Process Design and Optimization of State-of-th-Art Carbon Capture Technologies," Environmental Progress \& Sustainable Energy, vol. 33, pp. 993-999, 2014.

[8] A. Nandy, C. Loha, S. Gu, P. Sarkar and M. K. Karmakar, "Present Status and Overview of Chemical Looping Combustion Technology," Renewable and Sustainable Energy Reviews, vol. 59, pp. 597-619, 2016.

[9] M. M. Hossain and H. I. de Lasa, "Chemical-looping Combustion (CLC) for Inherent CO2 Separations- A Review," Chemical Engineering Science, vol. 63, pp. 4433-4451, 2008.

[10] S. Bayham, A. Tong, M. Kathe and L. S. Fan, "Chemical Looping Technology for Energy and Chemical Production," WIREs Energy Environment, vol. 5, pp. 216-241, 2106.

[11] S. Chen, L. Zeng, H. Tian, X. Li and J. Gong, "Enhanced Lattice Oxygen Reactivity of NiModified WO3-Based Redox Catalysts for Chemical Looping Partial Oxidation of Methane," Catalysis, vol. 7, pp. 3548-3559, 2017.

[12] J. RIley, R. Siriwardane, H. Tian, W. Benincosa and J. Poston, "Kinetic Analysis of the Interactions between Calcium Ferrite and Coal Char for Chemical Looping Gasification 
Applications: Identifying Reduction Routes and Modes of Oxygen Transfer," Applied Energy, vol. 201, pp. 94-110, 2017.

[13] H. Lim, D. Kang and J. Lee, "Phase Tranisition of Fe2O3-NiO to NiFe2O4 in Pervoskite Catalytic Particles for Enhanced Methane Chemical Looping Reforming-Decomposition with CO2 Conversion," Applied Catalysis B: Environmental, vol. 202, pp. 175-183, 2017.

[14] T. Pröll, K. Mayer, J. Bolhàr-Nordenkampf, P. Kolbitsch, T. Mattisson, A. Lyngfelt and H. Hofbauer, "Natural Minerals as Oxygen Carriers for Chemical Looping Combustion in a Dual Circulating Fluidized Bed System," Energy Procedia, vol. 1, pp. 27-34, 2009.

[15] P. Kolbitsch, J. Bolhàr-Nordenkampf, T. Pröll and H. Hofbauer, "Operating Experience with Chemical Looping Combustion in a $120 \mathrm{~kW}$ Dual Circulating Fluidized bed (DCFB) Unit," International Journal of Greenhouse Gas Control, vol. 4, pp. 180-185, 2010.

[16] C. Linderholm, M. Schmitz, P. Knutsson and A. Lyngfelt, "Chemical-Looping Combustion in a 10-kW Unit Using A mixture of Ilmenite and Manganese Ore as Oxygen Carrier," Fuel, vol. 166, pp. 533-542, 2016.

[17] C. Linderholm and M. Schmitz, "Chemical-Looping Combustion of Solid Fuels in a 10-kW Dual Circulating Fluidized Bed System Using Iron Ore as an Oxygen Carrier," Journal of Environmental Chemical Engineering, vol. 4, pp. 1029-1039, 2016.

[18] S. Luo, L. Zeng and L. S. Fan, "Chemical Looping Technology: Oxygen Carrier Characterisitics," Annual Review of Chemical and Biomolecular Engineering, vol. 6, pp. 53-75, 2015.

[19] L. Protasova and F. Snijkers, "Recent Developments in Oxygen Carrier Materials for Hydrogen Production via Chemical Looping Processes," Fuel, vol. 181, pp. 75-93, 2016.

[20] R. Sirwardane, E. Ksepko, H. Tian, J. Poston, T. Simonyi and M. Sciazko, "Interaction of Iron-Copper Mixed Metal Oxide Oxygen Carriers with SImulated Synthesis Gas Derived from Steam Gasification of Coal," Applied Energy, vol. 107, pp. 111-123, 2013.

[21] Y. Fan, R. Siriwardane and H. Tian, "Trimetallic Oxygen Carriers, CuFeMnO4, $\mathrm{CuFeMn} 2 \mathrm{O} 4$ and $\mathrm{CuFe} 0.5 \mathrm{Mn} 1.5 \mathrm{O} 4$ for Chemical Looping Combustion," Energy \& Fuels, vol. 29, pp. 6616-6624, 2015.

[22] R. Siriwardane, H. Tian, T. Simonyi and J. Poston, "Synergistic Effects of Mixed CopperIron Oxides Oxygen Carriers in Chemical Looping Combustion," Fuel, vol. 108, pp. 319333, 2013.

[23] M. Rydén, H. Leion, T. Mattisson and A. Lyngfelt, "Combined Oxides as Oxygen-Carrier Material for Chemical-Looping with Oxygen Uncoupling," Applied Energy, vol. 113, pp. 1924-1932, 2014. 
[24] P. Cho, T. Mattisson and A. Lyngfelt, "Comparison of Iron-, Nickel-, Copper-, and Manganese-Based Oxygen Carriers for Chemical Looping Combustion," Fuel, vol. 83, pp. 1215-1225, 2004.

[25] A. Evdou, V. Zaspalis and L. Nalbandian, "Ferrites as Redox Catalysits for Chemical Looping Processes," Fuel, vol. 28, pp. 367-378, 2016.

[26] Y. Fan and R. Siriwardane, "Novel New Oxygen Carriers for Chemical Looping Combustion of Solid Fuels," Energy \& Fuels, vol. 151, pp. 2248-2257, 2014.

[27] J. Adanez, A. Abad, F. Garcia-Labiano, P. Gayan and L. F. de Deigo, "Progress in Chemical-Looping Combustion and Reforming Technologies," Progress in Energy and Combustion Sciences, vol. 38, pp. 215-282, 2012.

[28] M. Tang, L. Xu and M. Fan, "Progress in Oxygen Carrier Development of Methane-based Chemical-looping Reforming: A Review," Applied Energy, vol. 151, pp. 143-156, 2015.

[29] L. Liu and M. R. Zachariah, "Enhanced Performance of Alkali Metal Doped Fe2O3 and Fe2O3/Al2O3 Composites as Oxygen Carrier Material in Chemical Looping Combustion," Energy \& Fuels, vol. 27, pp. 4977-4983, 2013.

[30] R. Siriwardane, H. Tian, D. Miller and G. Richards, "Fluidized Bed Testing of Commercially Prepared $\mathrm{MgO}$-promoted Hematite and $\mathrm{CuO}-\mathrm{Fe} 2 \mathrm{O} 3$ Mixed Metal Oxide Oxygen Carriers for Methane and Coal Chemical Looping Combustion," Applied Energy, vol. 157, pp. 348-357, 2015.

[31] A. Lyngfelt, "Chemical-looping Combustion of Solid Fuels - Status of Development," Applied Energy, vol. 113, pp. 1869-1873, 2014.

[32] A. Cabello, P. Gayán, A. Abad, L. F. de Diego, F. García-Labiano, M. T. Izauierdo, A. Scullard, W. G and J. Adánez, "Long Lasting Cu-based Oxygen Carrier Material for Industrial Scale in Chemical Looping Combustion," International Journal of Greenhouse Gas Control, vol. 52, pp. 120-129, 2016.

[33] L. Duan, D. Godino, V. Manovic, F. Montagnaro and E. Anthony, "Cyclic Oxygen Release Characteristics of Bifunctional Copper Oxide/Calcium Oxide Composites," Energy Technology, vol. 4, pp. 1171-1178, 2016.

[34] R. Siriwardane, W. Benincosa, J. Riley, H. Tian and G. Richards, "Investigation of Reactions in a Fluidized Bed Reactor during Chemical Looping Combustion of Coal/Steam with Copper Oxide-Iron Oxide-Alumina Oxygen Carrier," Applied Energy, vol. 183, pp. 1550-1564, 2016. 
[35] L. F. de Diego, F. García-Labiano, J. Adánez, P. Gayán, A. Abad, B. M. Corbella and J. M. Palacios, "Development of Cu-based Oxygen Carriers for Chemical-Looping Combustion," Fuel, vol. 83, pp. 1749-1757, 2004.

[36] W. Yang, H. Zhao, K. Wang and C. Zheng, "Synergistic Effects of Mixtures of Iron Ores and Copper Ores as Oxygen Carriers in Chemical-Looping Combustion," Proceedings of the Combustion Institute, vol. 35, pp. 2811-2818, 2015.

[37] K. Zhao, F. He, Z. Huang, A. Zheng, H. Li and Z. Zhao, "La(1-x)SrxFeO3 Perovskites as Oxygen Carriers for the Partial Oxidation of Methane to Syngas," Chinese Journal of Catalysis, vol. 35, pp. 1196-1205, 2014.

[38] A. Mishra, N. Galinsky, F. He, E. Santiso and F. Li, "Perovskite-structured AMnxB1-xO3 (A $=\mathrm{Ca}$ or $\mathrm{Ba}, \mathrm{B}=\mathrm{Fe}$ or $\mathrm{Ni}$ ) Redox Catalyst for Partial Oxidation of Methane," Catalysis Science \& Technology, vol. 6, pp. 4535-4544, 2016.

[39] E. Monazam, R. Breault, R. Siriwardane, G. Richards and S. Carpenter, "Kinetics of the Reduction of Hematite (Fe2O3) by Methane (CH4) during Chemical Looping Combustion: A Global Mechanism," Chemical Engineering Journal, vol. 232, pp. 478-487, 2013.

[40] E. Monazam, R. Breault, H. Tian and R. Siriwardane, "Reaction Kinetics of Mixed CuOFe2O3 with Methane as Oxygen Carriers for Chemical Looping Combustion," Industrial \& Engineering Chemistry Research, vol. 54, pp. 11966-11974, 2015.

[41] H. Leion, E. Jerndal, B. M. Steenari, S. Hermansson, M. Israelsson, E. Jansson, M. Johnsson, R. Thunberg, A. Vadenbo, T. Mattisson and A. Lyngfelt, "Solid Fuels in Chemical-Looping Combustion using Oxide Scale and Unprocessed Iron Ore as Oxygen Carriers," Fuel, vol. 88, pp. 1945-1954, 2009.

[42] M. Johansson, T. Mttisson and A. Lyngfelt, "Creating a Synergy Effect by Using Mixed Oxides of Iron- and Nickel Oxide in the Combustion of Methane in a Chemical-Looping Combustion Reator," Energy \& Fuels, vol. 88, pp. 2399-2407, 2009.

[43] A. Lambert, C. Delquié, I. Clémençon , E. Comte, V. Lefebvre, J. Rousseau and B. Durand, "Synthesis and Characterization of BImetallic Fe/Mn Oxides for Chemical Looping Combustion," Energy Procedia, vol. 1, pp. 375-381, 2009.

[44] M. Rydén, A. Lyngfelt, T. Mattisson, D. Chen, A. Holmen and E. Bjørgum, "Novel Oxygen-Carrier Materials for Chemical Looping Combustion and Chemical-Looping Reforming: LaxSr1-xFeyCo1-yO3-d Perovskites and Mixed Metal Oxides of NiO Fe2O3 and Mn3O4," International Journal of Greenhouse Gas Control, vol. 2, pp. 21-36, 2008.

[45] K. Ehrensberger, A. Frei, P. Kuhn, H. R. Oswald and P. Hug, "Comparative Experimental Investigations of the Water-Splitting Reaction with Iron Oxide Fe1-yO and Manganese Oxides (Fe1-xMnx)1-yO," Solid State Ionics, vol. 78, pp. 151-160, 1995. 
[46] P. Perreault, S. Rifflart, E. Nguyen and G. S. Patience, "Pyrolusite: An Alternative Oxygen Carrier for Chemical Looping Combustion," Fuel, vol. 185, pp. 630-638, 2016.

[47] S. Sajen, S. K. Singh, P. Mungse, S. Rayalu, K. Watanabe, G. Saravanan and N. Labhasetwar, "Mechanically Stable Mixed Metal Oxide of $\mathrm{Cu}$ and $\mathrm{Mn}$ as Oxygen Carrier for Chemical Looping Syngas Combustion," Energy \& Fuel, vol. 30, pp. 7596-7603, 2016.

[48] N. M. Pour, H. Leion, M. Rydén and T. Mattisson, "Combined Cu/Mn Oxides as an Oxygen Carrier in Chemical Looping with Oxygen Uncoupling," Energy \& Fuels, vol. 27, pp. 6031-6039, 2013.

[49] P. Mungse, G. Saravanan, T. Uchiyama, M. Nishibori, Y. Teraoka, S. Rayalu and N. Labhsetwar, "Copper Manganese Mixed Oxides: CO2-Selectivity, Stable, and Cyclic Performance for Chemical Looping Combustion of Methane," Physical Chemistry Chemical Physics, vol. 16, pp. 19634-19642, 2014.

[50] S. Sundqvist, M. Arjmand, T. Mattisson, M. Rydén and A. Lyngfelt, "Screening of Different Manganese Ores for Chemical-looping Combustion (CLC) and Chemical-looping with Oxygen Uncoupling (CLOU)," International Journal of Greenhouse Gas Control, vol. 43, pp. 179-188, 2015.

[51] E. R. Strobe, B. A. de Boer and J. W. Geus, "The Reduction and Oxidation Behavior of Manganese Oxides," Catalysis Today, vol. 47, pp. 161-167, 1999.

[52] K. Terayama and M. Ikeda, "Study on Thermal Decomposition of MnO2 and Mn2O3 by Thermal Analysis," Transactions of the Japan Institue of Metals, vol. 24, pp. 754-758, 1983.

[53] V. Frick, M. Ryden and H. Leion, "Investigation of $\mathrm{Cu}-\mathrm{Fe}$ and Mn-Ni Oxides as Oxygen Carriers for Chemical-looping Combustion," Fuel Processing Technology, vol. 150, pp. 3040, 2016.

[54] M. Ishida and C. Y. Wen, "Comparison of Zone-Reaction Model and Unreacted-Core Shrinking Model in Solid-Gas Reactions- I Isothermal Analysis," Chemical Engineering Science, vol. 26, pp. 1031-1041, 1971.

[55] M. Ishida, C. Y. Wen and T. Shirai, "Comparison of Zone-Reaction Model and UnreactedCore Shrinking Model in Solid-Gas Reactions- II Non-Isothermal Analysis," Chemical Engineering Science, vol. 26, pp. 1043-1048, 1970.

[56] Y. Park and O. Levenspiel, "The Crackling Core Model for the Reaction of Solid Particles," Chemical Engineering Sciences, vol. 30, pp. 1207-1214, 1975. 
[57] R. Pigford and G. Sliger, "Rate of Diffusion-Controlled Reaction Between a Gas and a Pourous Solid Sphere," Industrial and Engineering Chemistry, Process Design and Development, vol. 12, pp. 85-91, 1973.

[58] H. Sohn and J. Szekely, "A Structural Model for Gas-Solid Reactions with a Moving Boundary-III A General Dimensionless Representation of the Irreversible Reaction between a Porous Solid and a Reactant Gas," Chemical Engineering Science, vol. 27, pp. 763-778, 1972.

[59] P. Ramachandran and J. Smith, "A Single-Pore Model for Gas-Solid Noncatalytic Reaction," AIChE, vol. 23, pp. 353-361, 1997.

[60] M. Avrami, "Kinetics of Phase Change. I General Theory," Journal of Chemical Physics, vol. 7, pp. 1103-1112, 1939.

[61] M. Avrami, "KInetics of Phase Change. II Transformation- Time Relations for Random Distribution of Nuclei," Journal of Chemical Physics, vol. 8, pp. 212-224, 1940.

[62] M. Avrami, "Granulation, Phase Change, and Microstructure Kinetics of Phase Change III," Journal of Chemical Physics, vol. 9, pp. 177-187, 1941.

[63] Z. Qamar, A. Abad, T. Mattisson, B. Gevert and M. Strand, "Reduction and Oxidation Kinetics of Mn3O4/Mg-ZrO2 Oxygen Carrier Particles for Chemial-Looping Combustion," Chemical Engineering Science, vol. 62, pp. 6556-6567, 2007.

[64] M. Hossain and H. Lasa, "Reduction and Oxidation Kinetics of Co-Ni/A12O3 Oxygen Carrier Involved in a Chemical-Looping Combustion Cycles," Chemical Engineering Science, vol. 65, pp. 98-106, 2010.

[65] N. Muradov, "Hydrogen Via Methane Decomposition: an Application for Decarbonization of Fossil Fuels," International Journal of Hydrogen Energy, vol. 26, pp. 1165-1175, 2001.

[66] D. Gardner and C. Batholomew, "Kinetics of Carbon Deposition during Methanantion of CO," Industrial \& Engineering Chemistry Product Research and Development, vol. 20, pp. 80-87, 1981.

[67] J. F. Moulder, W. F. Stickle, P. E. Sobol, K. D. Bomben, E. J. Christian and R. C. King, Handbook of X-ray Photoelectron Spectroscopy: A Reference of Standard Spectra for Identification and Interpretation of XPS Spectra, Eden Pairie: Physical Electronics Inc., 1995.

[68] J. J. Goldstein, D. E. Newbury, P. Echlin, D. C. Joy, C. Fiori and E. LIfshin, Scanning Electron Microscopy and X-ray Microanalysis, Plenum, 1981. 
[69] R. H. Tien and E. T. Turkdogan, "Gaseous Reduction of Iron Oxides: Part IV. Mathematical Analysis of Partial Inernal Reduction-Diffusion Control," Metallurgical Transactions, pp. 2039-2046, 1972.

[70] S. Y. Chuang, J. S. Dennis, A. N. Hayhurst and S. A. Scott, "Development and Perforamnce of $\mathrm{Cu}$-Based Oxygen Carriers for Chemical-looping Combustion," Combustion and Flame, vol. 154, pp. 109-121, 2008.

[71] B. Wang, R. Yan, D. Lee, Y. Zheng, H. Zhao and C. Zheng, "Characterization and Evaluation of Fe2O3/A12O3 Oxygen Carrier Prepared by Sol-Gel Combustion Synthesis," Journal of Analytical and Applied Pyrolysis, vol. 91, pp. 105-113, 2001.

[72] K. Hall and J.-C. Yang, "Taconite Mining and Processing Industry Profile," U.S. Environmental Protection Agency, Rearch Triangle Park, 2001.

[73] W. Benincosa, R. Siriwardane, H. Tian and J. Riley, "Unique Phase Identification of Trimetallic Copper Iron Manganese Oxygen Carrier using Simultaneous Differential Scanning Calorimetry and Thermogravimetric Analysis during Chemical Looping Combustion Reactions with Methane," Applied Energy, vol. 203, pp. 52-534, 2017.

[74] T. Mattisson, M. Johansson and A. Lyngfelt, "The use of $\mathrm{NiO}$ as an Oxygen Carrier in Chemical-Looping Combustion," Fuel, vol. 85, pp. 736-747, 2005.

[75] "Charts \& Data; Charts \& Data for the Mining Industry," 17 July 2017. [Online].

[76] H. Lu, X. Shi, M. Costa and C. Huang, "Carcinogenic Effect of NIckel Compounds," Molecular and Cellular Biochemistry, vol. 279, pp. 45-67, 2005.

[77] A. Abad, J. Adanez, F. Garcia-Labiano, L. de Diego, P. Gayan and J. Celaya, "Mapping of the Range of Operation Conditions for $\mathrm{Cu}-, \mathrm{Fe}-$, and Ni-based Oxygen Carriers in Chemical-Looping Combustion," Chemical Engineering Science, vol. 62, pp. 533-549, 2007.

[78] M. Che and J. Vedrine, Characterization of Solid Materials and Heterogeneous Catalysts, Weinheim: Wiley-VCH Verlag \& Co, 2012.

[79] E. Monazam, R. Breault and R. Siriwardane, "Reduction of Hematite (Fe2O3) to Wüstite (FeO) by Carbon Monoxide (CO) for Chemical Looping Combustion," Chemical Engineering Journal, vol. 242, pp. 204-210, 2014.

[80] O. Levenspiel, Chemical Reactor Omnibook, Corvallis: OSU Book Stores, Inc., 1984.

[81] H. S. Fogler, Elements of Chemical Reaction Enginering, New Jersey: Pearson Education Inc., 2006. 
[82] F. Garcia-labiano, L. F. de Diego, J. Adanez, A. Abad and P. Gayan, "Reduction and Oxidation Kinetics of a Copper-Based Oxygen Carrier Prepared by Impregnation for Chemical-Looping Combustion," Industrial Engineering and Chemistry Research, vol. 43, pp. 8168-8177, 2004.

[83] M. I. Nasr, A. A. Omar, M. H. Khedr and A. A. El-Geassy, "Effect of Nickel Oxide Doping on the Kinetics and Mechanism of Iron Oxide Reduction," Iron and Steel Institute of Japan International, vol. 35, pp. 1043-1049, 1995.

[84] A. Abad, J. Adanez, A. Cuadrat, F. Garcia-Labiano, P. Gayan and L. F. de Diego, "Kinetics of Redox Reactions of Ilmenite for Chemical-Looping Combustion," Chemical Engineering Science, vol. 66, pp. 689-702, 2011.

[85] E. Monazam, R. Breault and R. Siriwardane, "Kinetics of Hematite to Wüstite by Hydrogen for Chemical Looping Combustion," Energy \& Fuels, vol. 28, pp. 5406-5414, 2014.

[86] L. F. de Diego, A. Abad, A. Cabello, P. Gayán, F. García-Labiano and J. Adánez, "Reduction and Oxidation Kentics of a CaMn0.9Mg0.1O3-d Oxygen Carrier for Chemical Looping Combustion," Industrial \& Engineering Chemistry Research, vol. 53, pp. 87-103, 2013.

[87] M. A. Stranick, "Mn2O3 by XPS," Surface Science Spectra, vol. 6, no. 1, pp. 39-46, 1999.

[88] F. Visentin, R. Gerbasi, G. Rossertto, C. De Zorzi, N. El Habra, D. Barreca and A. Gasparotto, "Fe2O3-TiO2 Systems grown by MOCVD: an XPS Study," Surface Science Spectra, vol. 18, no. 29, pp. 29-35, 2011.

[89] Y. Wang, Z. Wen, H. Zhang, G. Cao, Q. Sun and J. Cao, "CuO Nanorods-Decorated Reduced Graphene Oxide Nanocatalysts for Catalytic Oxidation of CO," Catalysts, vol. 6, no. 214, pp. 214:1-8, 2016.

[90] I.-J. Moon, C.-H. Rhee and D.-J. Min, "Reduction of Hematite compacts by H2-CO Gas Mixtures," Process Metallury, vol. 69, pp. 302-306, 1998.

[91] M. Brown, Handbook of Thermal Analysis and Calorimetry, Amsterdam: Elsevier.

[92] S. Vyazovkin and C. Wight, "Model-Free and Model Fitting Approaches to Kinetic Analysis of Isothermal and Nonisothermal Data," Thermochimica Acta, Vols. 340-341, pp. 53-68, 1999.

[93] S. Kurajica, A. Dezjak and E. Tkalcec, "Resolution of Overlapping Peaks and the Determination of Kinetic Parameters for the Crystallization of Multicomponent System from DTA or DSC Curves: I. Non-Isothermal Kinetics," Thermochimica Acta, vol. 288, pp. 123-135, 1996. 
[94] A. K. Galwey and G. M. Laverty, "The Nucleus in Solid State Reactions: Towards a Definition," Solid State Ionics, vol. 38, pp. 155-162, 1990.

[95] J. Adanez, A. Cuadrat, A. Abad, P. Gayan, L. F. de Diego and F. Garcia-Labiano, "Ilmenite Activation during Consecutive Redox Cycles in Chemical-Looping Combustion," Energy \& Fuels, vol. 24, pp. 1402-1413, 2010.

[96] K. Ewelina, P. Babinski and L. Nalbandian, "The Redox Reaction Kinetics of Sinai Ore for Chemical Looping Combustion Applications," Applied Energy, vol. 190, pp. 1258-1274, 2017. 